\title{
Grandparental investment: Past, present, and future
}

\author{
David A. Coall \\ School of Psychiatry and Clinical Neurosciences, University of Western \\ Australia, Fremantle, Western Australia 6160, Australia \\ david.coall@uwa.edu.au \\ http://www.uwa.edu.au/people/david.coall \\ Ralph Hertwig \\ Department of Psychology, University of Basel, 4055 Basel, Switzerland \\ ralph.hertwig@unibas.ch \\ http://www.psycho.unibas.ch/hertwig
}

\begin{abstract}
What motivates grandparents to their altruism? We review answers from evolutionary theory, sociology, and economics. Sometimes in direct conflict with each other, these accounts of grandparental investment exist side-by-side, with little or no theoretical integration. They all account for some of the data, and none account for all of it. We call for a more comprehensive theoretical framework of grandparental investment that addresses its proximate and ultimate causes, and its variability due to lineage, values, norms, institutions (e.g., inheritance laws), and social welfare regimes. This framework needs to take into account that the demographic shift to low fecundity and mortality in economically developed countries has profoundly altered basic parameters of grandparental investment. We then turn to the possible impact of grandparental acts of altruism, and examine whether benefits of grandparental care in industrialized societies may manifest in terms of less tangible dimensions, such as the grandchildren's cognitive and verbal ability, mental health, and well-being. Although grandparents in industrialized societies continue to invest substantial amounts of time and money in their grandchildren, we find a paucity of studies investigating the influence that this investment has on grandchildren in low-risk family contexts. Under circumstances of duress - for example, teenage pregnancy or maternal depression - there is converging evidence that grandparents can provide support that helps to safeguard their children and grandchildren against adverse risks. We conclude by discussing the role that grandparents could play in what has been referred to as Europe's demographic suicide.
\end{abstract}

Keywords: child development; demographic transition; empathy; grandmother hypothesis; grandparental investment; grandparental solicitude; intergenerational transfers; kin altruism; maternal depression; reciprocal altruism

\section{Introduction}

What is it about grandparents that is so lovely? I'd like to say that grandparents are God's gifts to children. And if they can but see, hear and feel what these people have to give, they can mature at a fast rate.

- Bill Cosby

You have to do your own growing no matter how tall your grandfather was.

— Irish proverb

The best babysitters, of course, are the baby's grandparents. You feel completely comfortable entrusting your baby to them for long periods, which is why most grandparents flee to Florida.

— Dave Barry (Babies and Other Hazards of Sex, 1984, p. 54)

From birth to adulthood children are gas-guzzlers. Across three traditional South American mixed-economy huntergatherer and horticulturist societies, Kaplan (1994) found that a child from birth to age 18 years consumes between 10 and 13 million more calories than the child itself produces. Who foots the bill? In cooperative breeding species such as humans, where child-rearing is not the exclusive domain of the parents, alloparents - that is, helpers other than the biological parents (Hrdy 1999;
David Coall is a Research Assistant Professor in the Community, Culture, and Mental Health Unit, School of Psychiatry and Clinical Neurosciences at the University of Western Australia. Still early in his research career, he has authored 11 publications across the fields of human biology, psychology, and pediatrics. $\mathrm{He}$ is especially interested in applying evolutionary theory to understand variation in human health and disease with a particular focus on intergenerational effects and the childhood environment.

Ralph Hertwig, Professor of Cognitive and Decision Sciences at the University of Basel, Switzerland, is the author of more than 75 publications in the areas of behavioral decision research and the methodology of social sciences. He received the German Society for Psychology's Young Scientist Award in 1996 and Early Career Award in 2006.

2009) - can step up. According to the cooperative breeding hypothesis, ancestral mothers evolved in groups "where a broad range of individuals - not just the genetic father - assist the genetic mother in protecting, carrying, or provisioning offspring, thereby permitting 
her to produce and rear costlier, slower-maturing offspring than otherwise would survive" (Hrdy 2005a, p. 69). Indeed, Kaplan observed that a child's "excessive" consumption is offset by adults who, between 20 and 40 years of age, produce an average surplus of 2,000 to 4,000 calories a day, which is generally maintained until 65 years of age (see also Kaplan 1997; Kaplan et al. 2000).

Who are these seeming altruists? Although it is still being debated whether the key sources of this and other help are fathers, grandmothers, grandfathers, siblings, or children (e.g., Hawkes et al. 1989; Hill 1993; Hrdy 1999; Ivey 2000; Kramer 2005a), both formal and informal models point to the evolutionary significance of intergenerational transfers (e.g., Bogin 1997; Hamilton 1966; Lee 2003a; 2008). Specifically, because individuals can increase their fitness by reproducing themselves or by helping kin who share common genes by descent (Hamilton 1964), kin selection theory (Maynard Smith 1964) predicts that it is most likely that these helpful individuals in the mother's social group will be close kin, such as children, siblings, uncles, aunts, and, crucially, grandparents. Indeed, although humans are similar to other cooperative breeding animals in many respects, it is the presence of knowledgeable, postreproductive helpers that sets them apart (Hrdy 2005b). During the prolonged postreproductive period, grandparents may have the opportunity and the motive to boost their own fitness by investing resources in their children's and grandchildren's survival and reproduction.

The opportunity for grandparents to care for their kin has never been as great as it is today. With increasing human life expectancy in industrialized societies - for example, in the United States, women's life expectancy in the 20th century has increased from 50.7 (born 1900) to 79.7 (born 2000) years (see Arias 2006) - today's grandparents' lives and those of their grandchildren overlap markedly, thus providing them with greater opportunity than ever before to play a significant role (Bengtson 2001). Empirical evidence supports the considerable role that grandparents play in taking care of their grandchildren. In Switzerland, for instance, it is estimated that unpaid grandparental childcare amounts to at least two billion Swiss francs per year (Bauer \& Strub 2002). In the United States, $28 \%$ of employed women rely on their parents or in-laws to provide childcare for their young children (Guzman 1999). Across ten European countries, 58\% of grandmothers and $49 \%$ of grandfathers provided some care for their grandchild during a 12-month period (Hank \& Buber 2009; similar percentages are found in the United States: Guzman 2004). A sample of Germans aged 55 to 69 years was observed to spend, on average, 12.8 hours each month supervising their grandchildren (Kohli et al. 2000).

Despite grandparents' historically unparalleled opportunity to care for their grandchildren, the impact of their investments may never have been smaller than it is today, at least when measured against classic components of fitness, such as number of offspring and child mortality. Specifically, with fertility rates below replacement and a delayed age at first childbirth in most industrialized societies, the probability of becoming a grandparent is falling. Moreover, low childhood mortality rates mean that the high-risk times of infancy - conditions under which grandparental investment may have evolved have largely been removed.
In light of these changes in grandparenthood in industrialized societies, we ask: Does the help that grandparents provide, which may have benefited grandchildren in traditional and historical populations, still yield benefits for grandchildren in industrialized societies? Our theoretical and empirical review in this article is structured as follows. Section 2 defines grandparental investment, spells out its underlying evolutionary rationale, and summarizes evidence from natural fertility societies. Section 3 turns to recent extensions of research on grandparental investment in terms of the notion of grandparental solicitude. Specifically, we review several likely determinants of grandparental investment across human populations. In Section 4 we describe economic and sociological accounts of grandparental investment. Sometimes in direct conflict with each other, the economic, evolutionary, and sociological accounts explain some of the empirical data, but none explain all. Section 5 examines a range of recent demographic changes that render the environment in which contemporary grandparents find themselves unique in human history, and that have profoundly altered the basic parameters of grandparental investment. In Sections 6 and 7, we review evidence addressing the issue of whether grandparental care, which benefited grandchildren in traditional and historical populations, still yields benefits for grandchildren in industrialized societies. Finally, in Section 8, we discuss public health and policy implications of grandparental investment.

\section{Grandparental investment: Definition, evolution, and evidence}

\subsection{What is grandparental investment?}

Resources invested in one's offspring are referred to as parental investment (see Geary 2000). Parental investment was originally defined as "any investment by the parent in an individual offspring that increases the offspring's chance of surviving (and hence reproductive success) at the cost of the parent's ability to invest in other offspring" (Trivers 1972, p. 139). Trivers' key point was that the currency of parental investment is its cost to the parent's ability to invest in other offspring. Therefore, the definition of parental investment was highly restrictive. However, a broader definition has evolved that incorporates:

any characteristics or actions of parents that increase the fitness of their offspring at a cost to any component of the parent's fitness ...., including any costs of parental care to the parent's subsequent mating success ... survival ... fecundity ... growth .... In addition, it is logical to include costs to fitness of other offspring or any ... other relatives. (CluttonBrock 1991, p. 9)

We interpret parental investment and, by extension, grandparental investment using this broader frame (see also Dawkins 2006; Geary 2000; Hertwig et al. 2002). Proxy outcome measures of parental investment and - to the extent that grandparents influence these outcomes grandparental investment that have been used include child mortality, birth weight, breast-feeding, infanticide, homicide, abuse, attachment/bonding between parents and infants, inheritance, interbirth interval, and educational investment (Hagen et al. 2006). The pathway through which grandparental investment affects a child's development can be direct (e.g., direct support, advice, 
or as a role model) and indirect via a third party (most often the grandchildren's parents).

\subsection{Why grandparental investment?}

Cooperative breeding species such as humans can increase their inclusive fitness directly by reproducing themselves or indirectly by helping their relatives, who share a proportion of their genotype by descent, to transmit their genes into future generations (Hamilton 1964). Grandparents can boost their inclusive fitness by devoting resources to their kin, helping to ensure the conception, birth, survival, growth, development, and reproduction of their relatives. The opportunity to increase indirect fitness is afforded by the long human lifespan in general, and in particular by the extensive postreproductive lifespan of human females that is unique among cooperative breeding animals (Hrdy 2005b). Although various forms of reproductive termination occur across a range of species (Cohen 2004; Packer et al. 1998; Paul 2005), humans are the only primate species in which reproductive termination is a distinct and universal trait (Caro et al. 1995; Pavelka \& Fedigan 1991). The survival of an organism beyond reproduction represents a biological anomaly: "[T]here should be little or no postreproductive period in the normal lifecycle of any species" (Williams 1957, p. 407). Attempting to explain this anomaly, Williams hypothesized that it would be adaptive to cease reproduction before death if the benefits to the woman's survival that is, removal of the risks associated with dying during childbirth, and the increased parenting effort that she could devote to her living children and grandchildren outweighed the benefits to reproductive fitness brought to the woman by further reproduction.

Couched in terms of "Hamilton's Rule" (Hamilton 1964), postreproductive helping behavior will evolve when an individual can help a related individual, who has a probability $r$ (i.e., coefficient of relatedness) of sharing this same gene by common descent, to survive and reproduce. This holds as long as the benefit $(B$, i.e., benefit to the relative receiving help), multiplied by the probability of sharing that gene $(r)$, outweighs the cost $(C)$ to the helper, in terms of his own survival and forgone reproductive opportunities. To wit, postreproductive helping behavior should evolve when $r B>C$. It is likely that this condition held for human ancestors because (a) grandparents are closely related to their grandchildren (grandparents, on average, share 25\% of their genes with each of their grandchildren, over and above the large proportion of genes that all humans share in any case; Dawkins 2006), (b) helping comes with a relatively low opportunity cost, in terms of the grandparent's own reproductive success (especially in the case of postreproductive grandparents), and (c) there is a potentially large benefit to grandchildren in terms of survival (and subsequent reproduction) during the high-risk times of infancy and childhood. ${ }^{1}$

Instigated by Williams' (1957) suggestion of the potential adaptive value of a postreproductive period, two related lines of reasoning have emerged, both emphasizing the vast investments required by the long childhood development period: the good-mother hypothesis and the grandmother hypothesis (reviews by Jamison et al. 2002; Peccei 2001a; Voland et al. 2005). ${ }^{2}$ The good-mother hypothesis holds that burdened with the long dependence of human children, a postreproductive period shields the mother from survival risks (i.e., pregnancy and giving birth) and the child from health risks (e.g., Down syndrome), and enables the mother to free up the resources to keep her children, particularly the youngest and most helpless, alive. This investment contributes to the mother's direct reproductive success by ensuring that her existing children survive. Thus, one would expect to see the presence of a mother and her increased longevity improving the survival chances and health state of her children, and we do. ${ }^{3}$

The grandmother hypothesis suggests that a postreproductive grandmother can foster her inclusive fitness by supporting her reproductive daughter and her grandchildren, thus increasing her daughter's fertility and improving her grandchildren's chances of surviving (e.g., Hrdy 1999). Moving the provisioning of children from exclusively the mother (i.e., breast-feeding) to allomothers, as the grandmother hypothesis proposes, has immediate reproductive consequences that could translate into inclusive fitness advantages for the allomothers (grandmothers): For example, ceasing to breast-feed terminates the mother's lactational amenorrhea (Ellison 1990), thus potentially reducing the interval to the daughter's next pregnancy. Comparative data confirm that humans wean their children at a younger age and have shorter interbirth intervals than do orangutans, gorillas, and chimpanzees (Hawkes et al. 1998; Kramer 2005a; Lancaster et al. 2000). The notion of the helpful grandmother, however, should not be conceptualized as selection to blindly maximize the number of offspring, because any increase in number of descendents is often accompanied by a decrease in investment per offspring and potentially survival (e.g., Coall et al. 2009; Gibson \& Mace 2005; Walker et al. 2008). Thus, we would expect to see the presence of a grandmother and her increased longevity improving the fertility of her daughter, but not at the expense of the survival and health of her grandchildren.

Although the good-mother hypothesis and the grandmother hypothesis, which are not mutually exclusive, predict a selective advantage associated with a postreproductive lifespan, attempts at modeling this advantage in contemporary, largely natural fertility, human populations have failed to support these hypotheses (e.g., Hill \& Hurtado 1991; 1996; Rogers 1993; see also Austad 1994). The benefits of early reproductive cessation in women diverting resources from reproduction to maternal care and avoiding the costs of continuing to reproduce - do not appear to outweigh the benefits of having additional children. However, consideration of additional costs, such as the decline in fertility with older age, increased perinatal mortality risk associated with giving birth at an older age, and increased competition with increased family size, as well as the variety of additional benefits earlier reproduction in daughters, improved growth and survival of grandchildren - suggests that the benefits of switching from reproduction to parenting could indeed outweigh the costs (Hrdy 2005a; Mace 2000; Turke 1997). Moreover, a recent analysis of data from two Gambian villages (1950-1975) showed that menopause may have a fitness advantage when the combined influence of both the increased maternal mortality associated with giving birth at an older age and the increased childhood 
survival associated with having a maternal grandmother present are taken into account (Shanley et al. 2007; see also Sear et al. 2000; Shanley \& Kirkwood 2001). Thus, the joint positive influence of a "good mother" and a "helpful grandmother" on survival during childhood may have created a selective advantage for postreproductive survival in women.

\subsection{Why postreproductive lifespan may not be adaptive}

In contrast to the aforementioned views, there are evolutionary theories proposing that the early cessation of reproduction, which implies the availability of postreproductive grandmothers, is an exaptation. It is not or was not originally adaptive (for reviews, see Peccei 2001a; Voland et al. 2005). On this view, menopause occurs because a woman has lived long enough to deplete the supply of primary oocytes with which she was born (Ellison 2001; but see Johnson et al. 2004; SkaznikWikiel et al. 2007). Several theories of senescence propose that small fitness benefits early in an individual's life will be selected for even if they exact a cost to fitness later in life. The reduced strength of natural selection at older ages permits these negative effects to remain unchallenged (Kirkwood 1977; Medawar 1952; Williams 1957). Therefore, rather than being advantageous itself, menopause may be a by-product of the adaptive benefit of producing, protecting, and storing a complete stock of primary oocytes at birth (Pavelka \& Fedigan 1991). Consistent with this interpretation is the similarity of human and chimpanzee ovarian follicle depletion rates (Jones et al. 2007; for gorillas see Atsalis \& Margulis 2008), which suggests that menopause may be a phylogenetic legacy and not unique to humans.

Even if this is the case, and menopause is a non-adaptive epiphenomenon that occurs because of humans' longevity, its presence creates the stage for helpful grandmothers. Bogin and Smith (1996) proposed a biocultural model in which menopause is non-adaptive but helpful grandmothers still play a central role. Once women started living past their reproductive ceiling, the only way that they could improve their inclusive fitness was by caring for their children and grandchildren. Whether human longevity and specifically the postreproductive longevity associated with menopause is adaptive or an epiphenomenon, grandparents are still in the right place, at the right time.

\subsection{Does grandparental investment enhance fitness? Evidence from natural fertility societies}

The influence of helpful grandmothers has been most intensively studied in historical and contemporary natural fertility societies with little or no access to modern medical care, including contraception. In these societies, grandparental investment matters for mothers' reproductive success. Sear and Mace's (2008) review of 45 studies investigating the presence of kin (father, grandparents, older siblings) supports the beneficial influence of postreproductive relatives: The presence of a maternal or paternal grandmother was associated with an increase in her grandchildren's probability of surviving in 69\% (9 of 13 studies) and in $53 \%$ (9 of 17 studies) of cases, respectively. For illustration, in their time series analysis of the Oromo agro-pastoralists of southern Ethiopia, Gibson and Mace (2005) found that the presence of a hardworking maternal grandmother, who helped the mother with heavy domestic tasks (e.g., grinding maize), increased the probability of a grandchild surviving to three years of age by $25 \%$. This effect is comparable to that achieved by installing a new water supply (Gibson \& Mace 2006).

Omnipresent grandparents, however, are not inevitably advantageous. Sear and Mace (2008) found evidence that the presence of grandparents has been associated with detrimental consequences for child survival. In 83\% (10 of 12 studies) of cases, the presence of maternal grandfathers had no effect, and in $75 \%$ the paternal grandfather had no effect or even a negative one on survival. There is currently little evidence supporting the adaptive significance of grandfathering (see Lahdenperä et al. 2007). Negative effects have also been reported for paternal grandmothers who may, in contrast to maternal grandmothers, increase their daughter-in-law's fertility at the expense of grandchild survival (Leonetti et al. 2005; 2007; Mace \& Sear 2005). For example, in a historical German population (1720-1874), the presence of a paternal grandmother increased a grandchild's risk of stillbirth by $35 \%$ (Voland \& Beise 2005) and infant mortality up to one month of age by $85 \%$ (Voland \& Beise 2002). The majority of these studies have been conducted in patrilineal societies; recent evidence from a matrilineal society suggests that even maternal grandmothers may have a negative effect under some circumstances (Sear 2008; but see Leonetti et al. 2005). The beneficial influence of grandparents cannot be taken as given. But there is evidence that the presence of maternal grandmothers in particular boosts the survival chances of their grandchildren.

\subsection{The issue of confounding effects}

The grandparental investment literature consists predominantly of correlational studies showing associations, or lack thereof, between grandparental presence and grandchild survival, for example. Of course, these studies do not show that this relationship is causal (Borgerhoff Mulder 2007; Sear \& Mace 2008). Some argue that shared genes or environment effects may equally account for the observed associations. Grandparents who live under good socioeconomic conditions or who have a fortuitous genetic complement are more likely to live long and healthy lives, and it would make sense that their grandchildren are more likely to survive, are healthier, or have better cognitive abilities (e.g., Modin \& Fritzell 2009). Although this possibility cannot be ruled out, several pieces of evidence speak against it. First, if the effect is due to shared genes or environment, one would expect that all grandparents who share the same proportion of genes by common descent or share their residence with a grandchild to have similar effects on grandchild survival. Clearly, this is not the case, as we discuss in the next section. Second, the detailed ethnographic accounts that accompany many correlational studies not only corroborate associations between kin presence and survival, growth, and development, but also highlight potential behaviors that promote these beneficial effects (e.g., Gibson \& Mace 2005; Leonetti et al. 2007). Third, many studies allow adjustment for a wide range of confounding variables (e.g., grandparental health, age, socioeconomic status, and residential distance) that at least partially 
control for shared environment effects (e.g., Borgerhoff Mulder 2007; Coall et al. 2009; Pollet et al. 2006). Taken together, this evidence suggests that it is unlikely that these effects can be explained away by shared gene or shared environment effects.

\section{Grandparental solicitude}

Grandparental helping behavior will not occur invariably, but, according to Hamilton's rule, is moderated by opportunity costs that may differ across types of grandparents (e.g., grandmother vs. grandfather). Indeed, one of the most robust findings across the grandparental investment literature is that maternal grandmothers invest the most, have most contact, and the closest relationships with their grandchildren, followed by maternal grandfathers, paternal grandmothers, and, finally, paternal grandfathers. ${ }^{4}$ Next to grandparent type, grandparental help is also likely to be a function of ecological conditions that determine the availability of grandparental resources, including the communities' subsistence strategy (e.g., the efficiency of food production), availability of relatives, marriage systems, residence patterns, inheritance patterns, and the health of relatives (reviewed by Sear \& Mace 2008). In what follows, we review five evolutionary factors that affect grandparents' inclination to invest their resources - genetic relatedness, paternity certainty, sex-specific reproductive strategies, reproductive value, and sex-biased grandparental investment - and two factors that influence the availability of resources family size and birth order.

\subsection{Genetic relatedness}

Hamilton's rule implies that the amount of resources transferred to an individual should depend on the relatedness of the provider ( $r$, the coefficient of relatedness). The closer the genetic relationship between two individuals, the more likely helping behaviors will evolve and the smaller the benefit needs to be for this altruism to be maintained (Hamilton 1964). There has been a long history of research suggesting that parents allocate their resources to children according to their genetic relatedness (e.g., Anderson 2005; Daly \& Wilson 1980; but see Hofferth \& Anderson 2003). Evidence that grandparents favor genetically related grandchildren also exists. The investment that grandparents make in a family reduces as the proportion of stepchildren in the family increases (Eggebeen 1992). Relatedly, relationships with stepgrandparents are not considered to be as close as those with biological grandparents (Aldous 1995). In lesbianmother families, grandchildren have more contact with the biological grandparents compared with non-biological grandparents (Patterson et al. 1998). Although the size of the variance accounted for in these studies and the necessity of a focus on genetic relatedness has been questioned (Rose \& Rose 2001), the patterns appear robust (see Anderson 2005).

\subsection{Paternity certainty}

Paternity uncertainty is the (perceived) risk that a male is not the biological father of his children. In contrast to males, because of internal fertilization mammalian mothers know categorically who their children are (Trivers 1972). In the few mammalian species in which males provide parental investment, they run the risk that their investment is being wasted on another male's offspring (Alexander 1974; Clutton-Brock 1991; Geary 2000; Trivers 1972). Both theoretical and empirical studies suggest that lower levels of paternity certainty are associated with reduced male parental care (see Anderson et al. 2007).

This logic can be extended to grandparents and their inclination to invest resources: Because the maternal grandmother is certain of her relationship with her daughter and her daughter's relationship with her grandchildren, it is predicted that maternal grandmothers will invest the most. Paternal grandfathers, on the other hand, are predicted to invest the least because there are two points of uncertainty between themselves and their grandchildren: They are not $100 \%$ certain of their relationship with their son nor of their son's relationship with their grandchildren. The maternal grandfather and paternal grandmother are indistinguishable between these two extremes and will both invest an intermediate amount, because they both have one point at which their relationship certainty with their grandchildren could be severed (Smith 1987; 1988).

Consistent with these predictions, across human societies maternal kin invest much more than paternal kin during the prenatal, delivery, and postnatal periods (Huber et al. 2004; Meehan 2005), and paternity uncertainty seems to be associated with reduced intergenerational investment from the father (Anderson et al. 2007; Kurland 1979) and the father's relatives (Gaulin \& Schlegel 1980; Hartung 1985). Yet, some caution should be taken in interpreting the effect of paternity uncertainty. Paternity certainty varies considerably across human societies (Anderson 2006). Moreover, throughout recent human history paternity certainty has increased substantially in many cultures (Gaulin 1980). Although the rebuttal to this statement is usually that paternity certainty was lower in our ancestral environment, and it is to this that humans are tuned, the findings that investment varies by paternity certainty within communities suggest that humans respond to local conditions. Other authors state that focusing on paternity certainty alone is inadequate (Euler \& Weitzel 1996; Pashos \& McBurney 2008) and that in patrilateral and patrilocal societies it is unable to account for the higher levels of investment by paternal relatives (Pashos 2000; but see Gibson \& Mace 2005).

\subsection{Sex-specific reproductive strategies}

Several authors have addressed the limits of paternity certainty by incorporating sex-specific reproductive strategies into their models of grandparental investment (Euler \& Michalski 2007; Euler \& Weitzel 1996; Huber \& Breedlove 2007). Because of the large energetic investment in the egg, as compared with the sperm, females are physiologically obligated to provide higher levels of parental investment than males (Bateman 1948). In mammals, internal gestation and the production of milk for offspring further increase this asymmetry (CluttonBrock 1989). Whereas females may focus on parental investment, producing a few high-quality offspring, males (because of their low levels of parental investment) 
can increase their reproductive success by mating with additional females. Therefore, investment put into female kin is more likely to be transformed into parental care, whereas resources invested in a son may be used for mating or parenting effort.

In light of this, individuals are assumed to be more inclined to invest in female relatives, whether providing practical help in the form of childcare or more social and psychological support (Euler \& Weitzel 1996; Euler et al. 2001). Extending this logic to grandparents, maternal grandparents who invest in their daughters and their children are expected to invest more than paternal grandparents who are investing in their sons and their sons children (Euler \& Weitzel 1996). In fact, this female kin bias in grandparental investment has been found across disciplines and societies (e.g., Gibson \& Mace 2005; Huber et al. 2004; Rossi \& Rossi 1990).

When combined with the notion of paternity certainty, sex-specific reproductive strategies further differentiate between the maternal grandfather and paternal grandmother. Because the maternal grandfather invests in his daughter's children, he invests more than the paternal grandmother who invests in her son's children (Euler \& Weitzel 1996). Evidence supports the significantly higher investment by maternal grandfathers compared with paternal grandmothers in the frequency of face-to-face interactions and emotional closeness (Hoffman 1980). Thus, the combination of paternity uncertainty and sexspecific reproductive strategies predicts the often-found pattern of grandparental investment where the maternal grandmother invests the most, followed by the maternal grandfather, the paternal grandmother, and the paternal grandfather (e.g., Bishop et al. [2009] and their summary of the data; but for different interpretations, see Huber \& Breedlove 2007; Pollet et al. 2006).

\subsection{Reproductive value}

An individual's age-specific future reproductive potential, the reproductive value, is another likely factor that influences how many resources he or she receives. If a parent or grandparent has the option of investing in his or her child who has just negotiated puberty and is beginning her reproductive career, or in that child's sibling who is equally related $(r=.5)$ but is approaching the end of her reproductive career, the child's higher reproductive value may strongly influence this decision. Relatedly, one would expect grandparents to transfer resources to grandchildren as a function of their reproductive value, with grandparents favoring those grandchildren who have a higher probability of reproducing in the future (Leek \& Smith 1991; Smith 1991; Smith et al. 1987).

Reproductive value may help us to understand, in part, why grandparents go to such enormous lengths and often self-sacrifice to support their teenage daughters who become parents. Individuals who have recently gone through puberty and are ready to begin their reproductive careers are at their peak reproductive value, which will gradually decline with age (Fisher 1930; Hamilton 1966; Williams 1957). If the aim is to maximize one's number of descendants in the future, preferentially investing in these high reproductive value young adults may be wise. Analyses conducted by Hughes (1988, as cited in Barrett et al. 2002, pp. 53-55) suggest that it is these young adults with high reproductive value who have the largest influence on any related individual's inclusive fitness. This influence is greater than that of any other segment of the community. Thus, the high cost that some grandparents incur in helping their children through teenage parenthood may be outweighed by the inclusive fitness benefits, both in contemporary and in ancestral environments.

\subsection{Sex-biased grandparental investment}

Under certain conditions, biasing investment preferentially towards sons or daughters may increase the probability that an individual will have grandchildren in the future (Trivers \& Willard 1973). In populations where members of one sex have higher potential reproductive success, grandparents may increase their inclusive fitness by preferentially investing in grandsons over granddaughters, or vice versa (see Euler \& Weitzel 1996; Jamison et al. 2002; Smith 1988). In theory, this may be possible to explore; however, studies examining this Trivers-Willard hypothesis in humans across just two generations have shown mixed results, and examining it over three generations will be even more challenging. To our knowledge, there is currently no convincing evidence of sex-biased grandparental investment in humans.

Table 1 summarizes our review of the evolutionary factors that can be expected to moderate grandparents' inclination to invest their resources. We now turn to two factors that are likely to affect the availability of grandparental resources.

\subsection{The availability of resources: Family size and birth order}

Actual investments presuppose the availability of resources, which, in turn, depends on numerous variables, such as the socioeconomic status of the grandparents and their state of health. Two factors - family size and birth order - are often incorporated into evolutionary analyses of parental and grandparental investments (e.g., Hagen et al. 2006; Kaplan 1994; Laham et al. 2005; Lawson \& Mace 2009; Leonetti et al. 2005), and both affect the amount of resources to be distributed.

Although larger families can recruit older siblings to provide resources for a family (Kramer 2002; 2005a), increased family size typically dilutes the resources available for each child (Blake 1987; Hertwig et al. 2002; Marks 2006) and grandchild (Laham et al. 2005; Leonetti et al. 2005; Uhlenberg \& Hammill 1998). In a study of 787 Australian university students, Laham et al. (2005) observed that the emotional closeness that a grandchild reported to his/her maternal grandfather or paternal grandmother depended on the availability of other kin (see also Smith 1988). Moreover, the general finding that maternal grandfathers provide more investment to grandchildren than do paternal grandmothers (Eisenberg 1988; Euler \& Weitzel 1996; Hoffman 1980; Pashos 2000) only held when the paternal grandmothers had other children in whom to invest.

Just as increased family size dilutes the resources available for each child and grandchild, so too does a higher birth order (Coall et al. 2009; Van Bavel 2006). Furthermore, there is good evidence that birth order has an additional nonlinear influence on the distribution pattern 
Coall \& Hertwig: Grandparental investment

Table 1. Factors associated with the inclination to invest grandparental resources (factors are derived using an evolutionary framework)

\section{Genetic relatedness}

1. The closer the genetic relationship is between two individuals, the more likely helping behaviors will evolve and the smaller the inclusive fitness benefit needs to be to maintain the helping behaviors (Hamilton 1964)

2. Because of the close genetic relationship between them, grandparents are inclined to invest in their grandchildren

\section{Paternity certainty}

1. Mammalian mothers know categorically who their children are because of internal fertilization. Males, however, are less than $100 \%$ sure who their children are (Trivers 1972)

2. Any father-child link in the relationship between a grandparent and grandchild reduces the certainty of their relationship below unity (Smith 1987; 1988)

Sex-specific reproductive strategies

1. Females are physiologically and psychologically obligated to provide higher levels of parental investment than males (Bateman 1948; Clutton-Brock 1989; Hrdy 1999)

2. Males, due to their lower levels of parental investment, can potentially increase their reproductive success by mating with additional females (Bateman 1948; Trivers 1972)

3. Due to these sex-specific reproductive strategies, resources invested in female kin are more likely to be used for parental care

4. Individuals are more inclined to invest in female relatives (Euler \& Michalski 2007; Euler \& Weitzel 1996)

5. Maternal grandparents who invest in their daughters and their daughters' children are expected to invest more than paternal grandparents who are investing in their sons (see Gibson \& Mace 2005; Huber et al. 2004; Rossi \& Rossi 1990)

6. Considering the grandparents' sex-specific reproductive strategies, grandmothers will invest more in their grandchildren than will grandfathers (Huber et al. 2004; Huber \& Breedlove 2007)

\section{Reproductive value}

1. Grandparents will be more inclined to invest resources in children and grandchildren who have a higher probability of surviving and reproducing in the future (Leek \& Smith 1991; Smith 1991; Smith et al. 1987)

2. Post-reproductive grandparents who have low reproductive value may invest more in their grandchildren than would relatives with the same coefficient of relatedness but higher reproductive value (e.g., aunts; Hames 1988)

\section{Sex-biased grandparental investment}

1. If there is a differential fitness return from investing in sons or daughters, parents and grandparents will be inclined to bias their investment to get the best fitness return from their investment (Trivers \& Willard 1973)

of parental and grandparental investment. Even if parents aim to invest equally in all of their offspring, an unavoidable unequal distribution of resources results due to the effects of birth order (Hertwig et al. 2002). The reason is that only the first and last born children have periods of investment that are not jeopardized by competition from siblings, thus undercutting middle-borns in a way that may have long-term consequences for family cohesion and relationships (Salmon \& Daly 1998; see review of evidence in Hertwig et al. 2002). By analogy, grandparents who invest equally in all of their grandchildren will also undercut middle-born grandchildren (but not so for bequests; see Bernheim \& Severinov 2000).

Last but not least, as grandparents are making the investment, grandparent's family size may matter as well. In a study of 580 grandparent-grandchild relationships, Coall et al. (2009) found that grandparent's family size (i.e., number of children and grandchildren) explained approximately three times more of the variance in grandparental investment (shared contacts, occasions to meet, discussions, activities, interests, and important roles) than grandchild's family size (i.e., number of siblings and birth order).

\section{Grandparental investment: Beyond kin-based explanations}

According to the dominant Darwinian explanation, grandparents can increase the representation of their genes in future generations by providing help to their children and grandchildren. Kin altruism, however, is only one of several possible accounts of grandparental investment. Within the social sciences, the two disciplines that have been most concerned with the topic of intergenerational transfers - of which grandparental investment is one manifestation - are economics and sociology. Next, we review economic and sociological accounts of grandparental investments and a Darwinian account that shifts the emphasis in explanations of grandparental investment from ultimate causes to proximate mechanisms.

\subsection{The economic view: Altruism versus mutual reciprocity}

In the economic literature, grandparental investment is typically treated as part of the same type of transfers along the intergenerational chain that characterizes parental transfers to children (although there are threegeneration variants of exchange, see e.g., Cigno 1993). Parental investment, in turn, has been an important subject of economic analysis at least since Becker (1974; 1991) and Barro's (1974) influential work on why parents care about the well-being of their progeny. Since then, however, as Arrondel and Masson (2006) noted, there has been an "impressive blossoming of miscellaneous models and motivations introduced in the literature" (p. 980), possibly due to the fact that the diverse 
manifestations of intergenerational transfers - for instance, post-mortem or inter-vivos (transfers among living members of the family), financial or time transfers, upward or downward transfers - are assumed to have different determinants and to serve different goals. Consequently, there is no single overarching model of parental, let alone grandparental, investment, although most models rest on the utility maximization and rational choice framework. Reviewing these manifold accounts of intergenerational transfers is beyond the scope of this article; moreover, such reviews already exist (e.g., Arrondel \& Masson 2006; Cigno 2006; Cremer \& Pestieau 2006; Laferrère \& Wolff 2006). Instead, we focus - at the expense of other motives (e.g., a "warm glow" associated with the very act of giving; Andreoni 1990) - on the two competing motives that a large proportion of economic models of intergenerational transfers have emphasized to explain inter-vivos intergenerational transfers: altruism and self-interested exchange.

Why do parents desiring to maximize their self-interest make enormous investments in their children? According to the Barro-Becker view, the solution to this puzzle is that a parent's welfare is influenced by the welfare of future generations. Formally, the parent's utility function is augmented by the child's likely lifetime utility (e.g., Barro 1974; Becker \& Tomes 1976; 1979), thus explaining why parents transfer resources to their children. Parents try to maximize total child quality as defined by the sum of their children's adult wealth. The model posits that parents allocate the resources as a function of child quality (e.g., the child's own skills and abilities), and later use wealth transfers to equalize outcomes across children (redistributive neutrality). Successive generations are linked by recursive altruistic preferences, that is, individuals have an altruistic concern only for their children, who in turn care altruistically for their children. Parents thus expect their children to adopt similarly altruistic behavior towards their children, and so on; thereby all generations of a family are linked together by a chain of intergenerational transfers.

According to the second motive, parents' transfers are less "pure," but part of a bargaining between parents and children (see Laferrère \& Wolff 2006). Transfers are a form of investment, like an investment into a portfolio, and the context of the investment is one in which parents anticipate that when they become old they will need help. Parents thus invest now (e.g., education expenses, gifts, loans) and in the future (promise of inheritance) to make their child behave altruistically toward them in the future - an intertemporal exchange between self-interested parents and children.

Both altruistic and exchange models face a number of empirical challenges, and according to Arrondel and Masson (2006), several surveys of the evidence have emphasized both models' "poor empirical performance" (p. 975). For the altruistic model, the challenges range from the empirical failure of the prediction that the amount of transfer should decrease with a child's income if the property of redistributive neutrality holds, to the fact that altruistic parents should transfer their wealth mainly in the form of gifts when children with limited liquidity need them most, rather than much later through bequests (Arrondel \& Masson 2006). For models of exchange, the crucial question is whether there exists a specific quid pro quo (intertemporal) exchange. If so, one would, for instance, expect that the levels of aid and attention that children give to their parents is positively correlated with their gift and inheritance expectations a prediction for which there is, at best, mixed evidence (Arrondel \& Masson 2006).

Few economic models of the altruism and exchange variety and beyond have explicitly considered grandparental investment (e.g., Wolff 2000), or treated it differently from a replication of the parent-child relationship. Exchange concerns may make the transfer motive most suitable for application to the grandparent-parent and grandparent-grandchild relationships, respectively. But again, there is little empirical evidence for a quid pro quo exchange. Using data from the German Aging Survey, Hoff (2007), for example, found that extensive financial transfers from grandparents to grandchildren existed, as did a small amount of instrumental support from grandchildren to grandparents; however, in only 10 out of $371(2.7 \%)$ cases was any exchange of resources evident. Only for the 80-85-year-old grandparents did the proportion of grandchildren providing instrumental support to grandparents exceed the proportion receiving financial transfers from them. The relative value of these time transfers is hard to evaluate (Arrondel \& Masson 2006) and may be crucial to the minority of grandparents that receives them; however, it is clearly outweighed by the preceding decades of their investment. ${ }^{5}$

\subsection{The sociological view: Values, norms, and institutions}

In sociology, the influential modernization paradigm has stressed the emergence of the nuclear family and the parallel weakening of bonds beyond the nuclear family. Probably owing to these predictions, the topic of grandparental investment has received scant attention from sociologists (Kohli 2004). This, however, changed in the closing years of the twentieth century, when demographic dynamics and economic pressure on state-funded oldage pension systems put the topic of intergenerational transfers and intergenerational solidarity (Monserud 2008; Wood \& Liossis 2007) on sociologists' scientific agenda. Since then, their inquiries have mostly turned to structural factors, social institutions, and cultural values that shape the process of intergenerational transfer. Structural factors relate to macro-level properties, such as female participation in the labor force, or income and wealth distributions. Social institutions relevant for transfer concern, for instance, laws that regulate testamentary freedom (e.g., in Germany the surviving spouse and children have a legal right to half of the estate, whereas in the United States there are virtually no constraints on the estate holder), and how wealth is taxed at death. Cultural values - underlying norms of reciprocity, family obligations, and responsibility - have been suggested as shaping intergenerational transfers (e.g., Finch \& Mason 1993). In a representative sample of the German population (the German Aging Survey), Kohli and Künemund (2003), for example, observed that retirees (>55 years) who agree to an unconditional solidarity norm ("If my family members need help, I will always be there") are much more likely to provide monetary transfers (to their kin during the 12 months prior to the survey) than those 
who espouse a strategic norm of direct reciprocity ("If I help my family members, I can expect help from them in return"). Closely related to investigations of such norms are studies of the different roles that grandparents can adopt (Kivett 1993), and the meaning of (Werner et al. 1998) and satisfaction with (Reitzes \& Mutran 2004) these roles.

As of now, the sociological inquiries have yielded a rich repertoire of empirical findings. On the one hand, it seems fair to say that this wealth of findings has not been born out of a strong theoretical framework, nor has it culminated in one, notwithstanding repeated calls from within sociology, and its crossover with psychology, for a theoretical synthesis (Smith \& Drew 2002; 2004; Szinovacz 1998b). On the other hand, sociologists' emphasis on variables and theoretical constructs (e.g., values and norms) that evolutionary researchers tend to neglect has revealed a number of key robust properties of intergenerational transfer and solidarity. Among these are the following: First, intergenerational support is not only a function of needs and the availability of resources, but also of the donor's (e.g., grandparent's) norms and values system (e.g., unconditional vs. conditional solidarity). Second, the development of public welfare systems - most profoundly implemented in European societies - has not crowded out support from beyond the nuclear family: There is a net flow of material resources from retirees to their adult children and grandchildren (Albertini et al. 2007). Third, for monetary resources, welfare state provision appears to enable rather than crowd out transfers (Attias-Donfut et al. 2005); furthermore, transfers in European countries to some extent depend on countries' welfare regimes (e.g., Albertini et al. 2007). Fourth, comparative analyses, for instance, between East and West Germany, have demonstrated that transfers from the elderly to their children occur even under unfavorable economic conditions (Kohli 2004).

Lacking an encompassing theoretical framework of grandparental investment, sociologists have made steps toward such a framework. One such step is the recent rational grandparent model (Friedman et al. 2008). It depicts grandparents as rational actors who use their relationship with their grandchildren as a conduit to a reciprocal relationship with the grandchildren's parents. That is, grandparents attempt to reduce the agonizing uncertainty regarding their quality of life in old age by investing differentially in the children of that child most likely, in the grandparent's perception, to reciprocate in the future. The mechanism assumed is that acts of investment in grandchildren activate children's norms of reciprocity. Clearly, this model shares aspects with other exchange theories (e.g., the indirect reciprocities model; Arrondel \& Masson 2006), but it also makes a number of unique predictions, some of which, however, are blatantly at odds with evolutionary theory, others with currently available evidence. For example, the model predicts, contrary to the kin altruism account of grandparental investment, that grandparents are indifferent as to whether their grandchildren are related to them or not (i.e., adopted). The model also assumes that to overcome the potential diffusion of responsibility among their children, grandparents must make investments "demonstrably and publicly differential" (Friedman et al. 2008, p. 42; these authors" emphasis). Such favoritism, however, is in conflict with both the parents' espoused value of equal treatment of children in egalitarian societies (Zervas \& Sherman 1994) and the "equal division puzzle" (Bernheim \& Severinov 2000); that is, the consistent observation that today's bequests are to a large extent distributed equally across all children within a family.

\subsection{An alternative Darwinian view: Evolutionary continuity and the empathy mechanism}

Recent years have seen sustained challenges to the standard categories of altruistic motivations in evolutionary theory and economics. According to those, people desire to enhance the welfare of others at a net welfare loss to themselves because they are related to those others (kin altruism); or because they expect future material benefits in repeated interactions (reciprocal altruism); or because they are concerned about building a reputation (reputation-based cooperation). Two bodies of evidence have challenged the sufficiency of these categories. The first body of evidence, gathered by experimental economists and psychologists, suggests that a substantial portion of people are strongly motivated by "other-regarding preferences" and are guided by concerns for fairness that cannot be easily reduced to the "weak reciprocity" that is motivated by long-term self-interest in repeated interactions.

Perhaps the most famous social game challenging the weak reciprocity view is the Ultimatum Game (Güth et al. 1982). In its simplest form, the Ultimatum Game involves a single round in which one person (the proposer) proposes to split a fixed pie (say \$100). The proposed split represents an ultimatum to the other person, the responder, who must accept or reject it. If the proposed distribution is accepted, it will be implemented. A rejection, however, results in a default payment - typically zero - for each player. Because of the extreme asymmetry in available actions, the equilibrium for this game involves very asymmetric payoffs: If the responder is self-regarding, she will accept any positive payoff in excess of the default value, even an offer of $\$ 1$, because it is better than nothing. Knowing this, a self-regarding proposer will offer just that, $\$ 1$. This prediction hinges on the assumption that the responder is rational and acts in a self-regarding manner, and that the proposer is convinced that the responder is rational and will act in a self-regarding manner. Conflicting with this prediction, a robust result in the Ultimatum Game, across hundreds of experiments, is that the vast majority of the offers to the responders are between 40 and $50 \%$ of the available pie (Fehr \& Schmidt 2006). Moreover, those proposals that offer the responder less than $20 \%$ of the pie are rejected with a substantial probability.

The most influential interpretation of this behavior which conflicts with the assumption made routinely by most economists (see Fehr \& Schmidt 2006) that material self-interest is the sole motivation of all people - has been in terms of altruistic punishment (Fehr \& Gächter 2002). Altruistic punishment means that individuals punish unfair and uncooperative behavior, notwithstanding the fact that the punishment is costly for them and yields no material gain. In the Ultimatum Game, the proposer is assumed to anticipate the risk of altruistic punishment both responder and proposer will receive nothing if the responder rejects the allocation - and thus offers a more equitable distribution than the equilibrium solution 
suggests. The threat of altruistic punishment of defectors has been suggested as a key condition of why humans, unlike other animals, are able to frequently cooperate with genetically unrelated strangers (i.e., no kin altruism), often in large groups, with people they will never meet again (i.e., no reciprocal altruism), and when reputation gains are small or absent (no reputation-based cooperation). Although altruistic punishment may be selectively disadvantageous at the individual level, it may be selectively advantageous at the group level (Wilson \& Wilson 2007), and thus could be the glue that keeps human societies together (e.g., Fehr \& Gächter). Moreover, neuro-scientific evidence indicates that, first, negative emotions towards defectors and, second, hedonic responses in reward-related brain areas when people cooperate or punish others for violations of widely accepted social norms (e.g., de Quervain et al. 2004; Sanfey et al. 2003) are the proximate mechanisms behind altruistic punishment.

Ethologists, primatologists, and psychologists have gathered the second body of evidence challenging the traditional categories of altruism. It suggests that precursory elements of key aspects of human moral behavior and other-regarding preferences, such as empathy, sympathy, consolation, perspective taking, theory of mind, and a sense of fairness, also exist in our closest primate relatives. De Waal (2006), in particular, has attacked what he calls the "veneer theory," according to which human morality is merely a fragile cultural layer, scarcely concealing the brutish natural tendencies that we have in common with other species. In contrast, he has argued that morality has evolved gradually in our forebears, especially in the great apes. The possibility of other-regarding preferences in nonhuman primates has triggered numerous fascinating studies of primates, and a heated debate. Some of these studies clearly support the notion of an evolutionary continuity between humans and primates with regard to crucial aspects of altruism (e.g., Brosnan \& de Waal 2003; Brosnan et al. 2005; Burkart et al. 2007; de Waal et al. 2008; Warneken et al. 2007), whereas others cast doubt on the existence of profound other-regarding preferences among primates in general and specifically in contexts involving food acquisition and distribution (e.g., Jensen et al. 2006; Silk et al. 2005; Vonk et al. 2007).

Possibly the single most important aspect of both of these roiling debates over the origins and the nature of human altruism for grandparental investment is the evolution of empathy. In several publications, de Waal (2008; Preston \& de Waal 2002) has proposed empathy to be a candidate mechanism that underlies directed altruism, which is altruistic behavior in response to another's pain, need, or distress. On this view, evolution may have selected empathy in animals as the main proximate mechanism to generate directed altruism. The mechanism, however, is not in opposition to the ultimate causes of behavior, but empathy-based altruistic acts are allocated in accordance with kin and reciprocal altruism theory (de Waal 2008, p. 279).

The empathy-mechanism thesis highlights the distinction between ultimate and proximate causes of behavior (Mayr 1961). Proximate mechanisms have received scant attention, with the kin altruism explanations of grandparental investment focusing instead on the investments' ultimate causes (reproductive success; section 2). According to de Waal's (2008) argument, the ultimate benefits of altruistic acts could be quite time-delayed, and thus unable to provide the motivational force necessary to implement acts of directed altruism. Empathy in terms of a rapid emotional connectedness mechanism, however, could bridge the gap between altruistic acts now and ultimate reproductive success benefits in the future. The core of the empathic capacity lies in the access that an organism gains to the subjective state of another organism through the subject's own neural and body representation. The mechanism works so well because it gives individuals an emotional stake in the welfare of others.

How did the empathy mechanism evolve? De Waal (2008) suggests that it "likely started in the context of parental care long before our species evolved" and "once the empathic capacity existed it could be applied outside the rearing context and play a role in the wider network of social relationships" (p. 282). Via the empathy mechanism, de Waal puts altruism back into altruism. Analogously, via the empathy mechanism one could also put altruism back into grandparental altruism. That is, the proximate cause for why grandparents care for the welfare of their children and grandchildren is not that they bet on return benefits of altruistic behavior, but may lie in their capacity for empathy, or in Hrdy's (2009) term, in humans' hypersociality. Empathy alerts them to their kin's needs, helps them to identify emotionally with them, and provides them with psychological benefits (e.g., satisfaction) from giving support.

\subsection{Conclusion}

What can we do with the multiple theoretical views on altruism in general and human grandparental investment in particular? In our view, the task for the future must not be to play them off against each other and declare one the winner. Grandparental investment can be analyzed and explained on multiple levels of description. Kin altruism, with its focus on fitness consequences, is key in understanding its evolution and in identifying a set of variables (Table 1) that explain some of the observed variance in grandparental investments. Kin-based accounts, however, have passed over the proximal causes, and left unregarded the motivational engine behind grandparents' altruistic acts. Even if, in the case of grandparental investment, there is no long delay between altruistic grandparental acts (e.g., babysitting) and their reproductive success, the question is whether grandparents muster their continuous motivation to care for their family's welfare by contemplating progeny or by proximate motivational mechanisms that are robust and disconnected from its ultimate goal.

Just as de Waal's new Darwinian view stresses proximate motivational mechanisms, sociological theories can be seen as revealing proximate motivational mechanisms by explaining how individuals' norms and values (e.g., conditional vs. unconditional altruism) affect the degree to which a grandparent cares. Moreover, interindividual differences in norms and values can help to discern which grandparents are better modeled in terms of the Barro-Becker kind of altruism and which grandparents can be modeled as partaking in a strategic bargaining game. Relatedly, the emotional connectedness mechanism or empathy mechanism could provide the psychological foundation for the BarroBecker kind of parental and grandparental altruism. 
Beyond these proximate levels, the sociological and economic theories consider grandparental investment and intergenerational transfers from a macro perspective that is missing in all Darwinian accounts. How are grandparental investments influenced by institutional arrangements (e.g., laws of inheritance)? Does a state-financed welfare system crowd out grandparental investment? An institutional perspective also highlights that intergenerational transfers are not necessarily unidirectional. To the extent that public pension systems are (partly) financed through a pay-as-you-go scheme (e.g., Germany; BörschSupan \& Miegel 2001), enormous tangible resources are also transferred from younger to older generations.

All of the theoretical views are valuable insofar as they focus on, analyze, and explain important yet different aspects of grandparental investment. Yet, at the same time, each view is deficient because of its narrow focus. Like in the Indian tale of the blind men and the elephant - in which each blind man touches a different part, but only one part, say, the tusk, to learn what the elephant is like, thus arriving at very different descriptions of the animal - each approach considers one of several aspects: ultimate causes, proximate mechanisms, values or institutions, welfare regimes, and so on. The task for the near future is to seriously attend to the other disciplines' views: Consider how rarely an article on grandparental investment published in an evolutionary journal cites sociological work, and vice versa. The task for the medium term is to work toward a comprehensive framework that integrates the different aspects of grandparental investments reviewed here. Such an integrative framework and interdisciplinary research program is timely. The way parental and grandparental investments, public and private transfers are institutionally organized and incentivized will be increasingly important in post-demographic transition societies where human capital becomes an ever smaller resource. Indeed, the link between grandparental investments and demographic trajectories is the topic of our next section.

\section{A paradoxical new world: So much to share, so little impact?}

According to demographic transition theory (e.g., Caldwell et al. 2006), societies progress from a pre-modern regime of high fertility and high mortality (Stage 1) to a postmodern regime of low fertility and low mortality (Stage 4), and possibly, according to some theorists, to a Stage 5 , in which birth rates fall below death rates, thus causing the population to decline (but, for more optimistic recent findings, see Myrskylä et al. 2009). At this point, most industrialized societies are in Stage 4, and most of the radical demographic and social transformation has occurred over the last 150 years. A wide range of factors has contributed to this transition, including progress in medical science, technology, the rise in productivity and income, the availability of contraceptive technologies, rising female participation in the labor market, increasing costs of having children (e.g., education), and new values that justify lower fertility, and even childlessness.

The conditions under which the beneficial role of grandparents may have evolved are still maintained in the few contemporary pre-industrialized small-scale societies that have not begun the demographic transition: short life expectancy, high fertility and infant mortality, and the wealthy out-reproducing the poor. In contrast, in post-demographic transition societies life expectancy is long, fertility and infant mortality are low, and the poor now out-reproduce the wealthy (Borgerhoff Mulder 1998; Lee 2003b). In what follows, we describe how these demographic changes affect basic parameters of grandparental investment in industrialized societies.

\subsection{Shared lifespan}

The recent increase in human life expectancy has expanded the shared lifespan between grandparents and grandchildren, thus creating theoretically an ideal situation for grandparents to impact their grandchildren's welfare. Using cohort-specific survival rates and age-atfirst-birth data from Switzerland, Höpflinger et al. (2006) estimated that of the grandchildren who reached 20 years of age in 1900, $27 \%$ would have had one or more living grandparents. This figure had risen to $92 \%$ in 2000 . At 35 years of age, only $1 \%$ of grandchildren would have had at least one surviving grandparent in 1900; in the year 2000 , even at 35 years of age, $39 \%$ of grandchildren would have had one or more surviving grandparents (for U.S. data, see Uhlenberg 1996).

How will this trend continue in the future? Using mortality, fertility, and average age at first birth data for England and Wales, Murphy and Grundy (2003) estimated the proportion of people born between 1911 and 2050 with living mothers and women with living children. From the grandparent's point of view, the future looks bright. A combination of the decline in age at first childbirth to a low in the 1970s and steadily declining mortality rates means that $60 \%$ of 70 -year-olds currently have two or more living children and by extension, potentially grandchildren. It is estimated that this proportion will remain above $50 \%$ until at least 2030. From the grandchildren's point of view, we may be about to leave a golden age of shared lifespan. A combination of an increasing age at first childbirth since the 1970s and a less marked decline in mortality rates means that it is more likely a mother will be dead for a given age of her children (and grandchildren). Therefore, the proportion of adults with a living mother, and thus the proportion of grandchildren with a living grandmother, has started to decline from those individuals born around 1970. In terms of human history, grandparents and grandchildren may currently be at the zenith of their shared lifespan.

More generally, even though the average age at first childbirth is rising (Billari et al. 2007), and hence delaying the onset of grandparenthood, in industrialized societies the typical grandparent is young (relative to life expectancy) and healthy (Manton et al. 2008). For example, Schwartz and Waldrop reported in 1992 that nearly 50\% of grandparents in the United States were aged less than 60 years, 33\% less than 55 years, and only 20\% older than 70 years. Some have argued that healthier grandparents are more likely to be pursuing their own, perhaps costly, interests outside their family (e.g., traveling). However, in industrialized societies, healthier and younger grandparents have been found to invest more in their grandchildren (Höpflinger et al. 2006; see also Euler \& Weitzel 1996), and to increase the chance of 
grandchildren surviving in historical populations (Lahdenperä et al. 2004).

\subsection{Low fertility rates}

According to Kohler and colleagues, "The majority of the world's population is living in countries with near-replacement or below-replacement fertility" (Kohler et al. 2002, p. 641). In Europe, for instance, Turkey is the only country among 46 countries studied where generation replacement is guaranteed (with a fertility level higher than 2.1 children in 2002; Council of Europe 2005). At the turn of the millennium, 14 countries in Europe had a total fertility rate below 1.3, which implies halving of the stable population size every 45 years (Kohler et al. 2002). As a consequence, the probability of becoming a grandparent is falling, and those people who do become grandparents are likely to have fewer biological grandchildren, relative to the past.

\subsection{Increased individual wealth and investment per child}

The influence that grandparents, like parents, have on the development of grandchildren is largely dependent on resource availability (see Borgerhoff Mulder 2007; Gibson \& Mace 2005; Hadley 2004; Lawson \& Mace 2009). In industrialized societies, ensuring that descendants' skill base and wealth makes them competitive, means that the cost of raising children has increased exponentially (see Borgerhoff Mulder 1998; Kaplan 1996; Mace 1998). Thus, even though low childhood mortality and fertility rates may translate into fewer, wealthier descendants, the need for grandparental investment may not necessarily diminish. Rather, the increased cost of children may further exacerbate the demands on grandparents. Consistent with this interpretation, higher socioeconomic status grandparents invest more in family members (Cao 2006) and show higher levels of affectual solidarity with their grandchildren (Wood \& Liossis 2007). However, the aspect of socioeconomic status being investigated (e.g., income or education) and the form of investment being made (e.g., time or money) may strongly influence the association between grandparental wealth and investment.

In industrialized societies, higher socioeconomic status groups tend to delay reproduction. Even people who want to have children often consciously delay reproduction to accumulate resources (Hammarberg \& Clarke 2005). Increasingly, people are turning to reproductive technologies to assist conception later in life (Oakley et al. 2008). The fact, however, that most industrialized countries cannot match demand for fertility treatments (Hoorens et al. 2007), and the increased risk of poorer outcomes for the mother and child associated with such technologies (Fisher et al. 2005; Kalra, \& Molinaro 2008), means that the probability of becoming a grandparent continues to fall, and that those people who become grandparents may need to provide especially high levels of investment.

To conclude, grandchildren and grandparents have never had and may never again have more shared lifetime than today. Moreover, intergenerational transfers remain a robust property of grandparent-grandchild relationships in industrialized societies, although they are dependent on a range of factors such as grandparents' socioeconomic status, their role within the family, grandparents' occupational status, and the presence of elderly parents to care for in addition to children and grandchildren (e.g., AttiasDonfut \& Segalen 2002; Cooney \& An 2006). One likely consequence of the substantial increase in shared lifespan and the lower birthrate is that the time, money, and affection that grandparents invest is spread across fewer grandchildren, increasing the potential investment per grandchild. Paradoxically, however, their investments are likely to be less beneficial than ever - at least when measured in terms of fitness consequences. Does that mean that grandparental investment has become a vain endeavor?

\section{Grandparental investment in industrialized societies: Any evidence for beneficial effects under low-risk conditions?}

Lack of impact on classic fitness indicators in industrialized societies does not mean that grandparental investment has lost all significance. Rather, the question is on which other dimensions may one find evidence of potential benefits, inasmuch as they exist? Even in affluent societies, in which, typically, children receive medical care, do not starve to death, and learn to read and write, childhood experiences, for example, in terms of the availability of monetary, cognitive, social, and emotional resources, vary enormously. Reflecting such variation in the United States, the Gini coefficient - a classic tool for measuring inequality - climbed steadily from 0.395 in 1974 to 0.47 in 2006, before dipping slightly to 0.463 in 2007. In Britain, the Gini (and thus inequality) has risen from 0.25 in 1979 to 0.35 in 2006 (The Economist, April 2, 2009).

In light of heterogeneity in childhood experiences and grandparents' ability to provide a buffer against psychological and environmental challenges (Cohen \& Wills 1985; Uhlenberg 2009), one hypothesis is that the beneficial effects of grandparental investments in industrialized societies reside in "softer" dimensions, such as the grandchildren's cognitive and verbal ability, mental health, and well-being. However, according to another hypothesis, it is far from clear whether the unique environments that grandparents experience in industrialized societies necessarily support the contact, family structures, and relationships that are available in contemporary traditional societies and historical populations (see, e.g., Denham \& Smith 1989). As a consequence, the impact of grandparents on children's development may lack a systematic pattern and, literally, "may be beneficial, harmful, or neutral" (Denham \& Smith 1989, p. 348).

To evaluate which of these two hypotheses is more appropriate, we search for evidence in two stages. We first focus on the impact that grandparents have on the development of their grandchildren in low-risk family contexts. Our goal is to determine those effects of grandparental investment that emerge when no unusual risks shape the grandchild's development. Second, we turn to the effects of grandparental investment in two highrisk family contexts, namely, teenage pregnancies and maternal depression, and we aim to describe what may be called the buffering effects of grandparental investment under conditions of duress. 


\subsection{Low-risk family contexts: Grandparental investment and the physical and mental well-being of children and grandchildren}

Could the beneficial influence of grandparents express itself in industrialized societies in terms of successful development on various psychological dimensions? In search of an answer, we conducted a systematic review of the grandparental investment literature. Empirical studies examining the influence of grandparents on their grandchildren have been conducted in sociology, psychology, medicine, biology, economics, and education. To identify these studies, we searched seven databases: PsychINFO (1970 2007), Medline (1970-2007), ERIC (1970-2007), Sociological Abstracts (1970-2007), EconLit (1970-2007), Biosis Preview (1980-2007), and ISI Web of Science (1970-2007), limiting the search to English-language journal articles. The same 11 searches were conducted in each database. The key words "grandparent" and "grandchild" were searched in the title, abstract, and keywords fields in each database, in combination with terms focusing on a range of measures reflecting childhood development: "development," "verbal ability," "verbal scores," "SAT scores," "academic performance," "school performance," "grade point average," "cognitive performance," "cognitive ability," "adjustment," and "behavioral development." This search was completed in December 2007.

All searches were cross-matched for duplication, yielding 196 publications. We read the title and abstract of each study to identify those that could be relevant. If there was any indication that grandparental investment (in terms of time, money, care, contact, etc.) in industrialized societies was empirically studied, we read the complete article. We then culled this group, keeping only those articles that (a) provided direct measures of grandparental investment (rather than indiscriminate extended kinship investment), (b) provided direct measures of child outcome (e.g., SAT scores, school performance, grade point average, behavioral development), and (c) investigated a low-risk family context in industrialized societies. Because of the low-risk criterion, we excluded studies involving three-generation households, households where grandparents were the primary caregivers, and households where the influence of grandparents was examined following parental divorce - circumstances that typically represent higher risk family situations, in which grandparents and their families are often disadvantaged themselves (Brandon et al. 2007; Lavers \& SonugaBarke 1997). When one of the articles cited a study that appeared relevant, we looked it up and included it if the study met the described criteria; similarly, we looked up all studies that cited the article (using Web of Science).

This produced only 13 publications, and, of these, merely four publications met all of our criteria: Tinsley and Parke (1987), Falbo (1991), Scholl Perry (1996), and Fergusson et al. (2008). ${ }^{6}$ Three features of the literature may account for why our search identified so few studies. The first is that very few studies provide direct measures of grandparental investment, but focus instead on outcomes, such as educational attainment as proxies for grandparental investment. The second feature is that many studies focus on co-residence, which occurs more frequently with non-intact families (high-risk context), but is generally low in countries such as the United
States (Hill 2006). Finally, many studies investigate the impact of help from the broad category "other adults," rather than specifically grandparents (e.g., Runyan et al. 1998; Surkan et al. 2007). We now turn in more detail to the few relevant studies that we retrieved.

6.1.1. Infant physical and mental development. Tinsley and Parke (1987) investigated measures of physical and mental development as a function of the frequency of grandparent-grandchild contacts in a sample of 30 seven-monthold Caucasian infants and their families in the United States. All of the infants were healthy and none of the grandparents were daily caregivers to their grandchildren, lived in the same household, considered themselves retired, or lived more than 50 miles $(80 \mathrm{~km})$ away from their grandchildren. Each parent and grandparent was observed playing with the infant for five minutes, and adult-infant interaction and adult global behaviors were rated. To measure grandparental investment, parents completed a social support questionnaire and grandparents completed a questionnaire measuring their contact (frequency) with their grandchildren. Finally, the Bayley Scales of Infant Development, administered on a separate home visit, measured the infant's mental and physical development.

Table 2 shows the statistically significant relationships between grandparent-infant patterns of interaction and infant development scores, and reports the effect size measure eta ${ }^{2}\left(\eta^{2}\right)$. First, grandchildren whose grandparents engaged in more stimulating and engaging behavior had higher Bayley scores across both the physical and mental domains (only significant effects were reported). All significant effects were positive and of a large size. Second, infants who had high levels of contact with their grandparents had higher Bayley Mental Development Index scores compared with infants with low contact $\left(\eta^{2}=0.148\right.$ and 0.146 , respectively). There was no influence, however, on the Bayley physical development scales.

To the best of our knowledge, Tinsley and Parke (1987) were the first to show that more stimulating and interactive play and more contact with grandmothers and grandfathers (but without differentiating between lineages) have positive influences on infant mental development. However, from their study, it is not possible to distinguish between direct influences of grandparenting and indirect influences via parenting (see Bridges et al. 2007), and thus to distinguish between predictions from the goodmother and grandmother hypotheses. In sum, these results document a suggestive, not necessarily causal, association between grandparents' behaviors and infant mental development that deserves further investigation.

6.1.2. Academic achievement. In a sample of 1,460 Chinese grandchildren, Falbo (1991) analyzed the relationship between grandparent-grandchild contact and the child's language and mathematics test scores during primary school. The quality of contact was measured in terms of the frequency of contact (three-point scale from "rarely together" to "often together"), multiplied by each grandparent's highest educational attainment (seven-point scale from "illiterate" to "graduate degree"). For both language and mathematics across all types of grandparents, Falbo found a positive relationship of small effect size $\left(r^{2}=.01-.05\right)$. However, because contact with grandparents and grandparents' education were not separated, and 
Coall \& Hertwig: Grandparental investment

Table 2. Relationships between grandparent-infant interaction patterns (median split) and infant development scores (adapted from Tinsley \& Parke 1987) ${ }^{a}$

\begin{tabular}{|c|c|c|}
\hline Grandparent-infant dyad & $\begin{array}{l}\text { Observed grandparent-infant } \\
\text { interaction patterns }\end{array}$ & Infant development score ${ }^{\mathrm{b}}$ (effect size $\left.{ }^{\mathrm{c}}\right)$ \\
\hline \multicolumn{3}{|l|}{ Grandmother-infant } \\
\hline & Higher positive verbalizations & Higher Bayley raw Mental Health Index $\left(\eta^{2}=0.138\right)$ \\
\hline & Higher demonstrating without a toy & $\begin{array}{l}\text { Higher Bayley raw }\left(\eta^{2}=0.184\right) \text { and adjusted Physical Health } \\
\text { Index }\left(\eta^{2}=0.173\right)\end{array}$ \\
\hline & More kissing & $\begin{array}{l}\text { Higher Bayley raw }\left(\eta^{2}=0.155\right) \text { and adjusted Physical Health } \\
\quad \text { Index }\left(\eta^{2}=0.143\right)\end{array}$ \\
\hline & Highly imaginative & $\begin{array}{l}\text { Higher Bayley raw and adjusted Physical Health Index (both } \\
\eta^{2}=0.190 \text { or greater) }\end{array}$ \\
\hline & Highly confident & Higher Bayley adjusted Physical Health Index $\left(\eta^{2}=0.164\right)$ \\
\hline \multicolumn{3}{|l|}{ Grandfather-infant } \\
\hline & Highly responsive & $\begin{array}{l}\text { Higher Bayley raw and adjusted Physical Health Index (all } \\
\eta^{2}=0.144 \text { or greater) }\end{array}$ \\
\hline & Highly playful & $\begin{array}{l}\text { Higher Bayley raw and adjusted Physical Health Index (all } \\
\eta^{2}=0.144 \text { or greater) }\end{array}$ \\
\hline
\end{tabular}

${ }^{a}$ Unfortunately, only those relationships found to be statistically significant were presented in the original text and are reproduced here. It is likely that a broad range of unreported small and medium effects also exist.

${ }^{\mathrm{b}}$ The Bayley Scales of Infant Development measured infant mental and physical development across cognitive, motor, and behavioral domains. This produced four scores: (a) a raw score on the Bayley Mental Scale, (b) an adjusted Bayley Mental Development Index, (c) a raw score on the Bayley Physical Scale, and (d) an adjusted Bayley Physical Development Index.

${ }^{c}$ The original text reported the $F$ statistics, therefore the effect size calculated was eta ${ }^{2}\left(\eta^{2}\right.$; Cohen 1965, p. 105). Eta ${ }^{2}$ can be interpreted as the proportion of variance in the dependent variable that is attributable to each effect $\left(\eta^{2}\right.$; small $[0.01]$, medium [0.06], and large [0.14] effects; Cohen 1988):

$$
\eta^{2}=\frac{F^{*} d f_{\text {effect }}}{F^{*} d f_{\text {effect }}+d f_{\text {error }}}
$$

grandchildren from nuclear and three-generation families were examined together, it is unclear how much of this effect reflects grandparental investment.

Scholl Perry (1996) also investigated the influence of grandparental investment on academic achievement in an ethnically diverse sample of 75 middle-upper class adolescents (14-17 years of age) from New York State. Grandparental investment was measured in terms of the grandparent-grandchild contact (frequency), their emotional closeness, and social distance. Children's academic achievement was measured through students' self-reported grade point average. The main results were: First, the social distance to grandfathers, but not grandmothers, was associated with grade point average. Specifically, students who reported a larger social distance between themselves and their paternal grandfather had higher grade point averages $(d=0.95)$. Conversely, there was a trend towards the opposite effect in maternal grandfathers: Smaller social distance was associated with higher grade point average $(d=0.62$; Cohen's $d$ is interpreted as a standardized mean difference between two scores of a small [0.2], medium [0.5], or large [0.8] size; see Cohen 1988).

Scholl Perry's (1996) analysis suggests that grandfathers from different lineages may have different influences on their grandchild's academic achievement, consistent with a matrilateral kin bias predicted by evolutionary theorizing (see Table 1). Moreover, grandchildren's reported social distance mirrored the predicted role of grandparents' lineage and gender, with the smallest reported social distance (closest) to maternal grandmothers, followed by maternal grandfathers, then paternal grandmothers, and the largest social distance to paternal grandfathers.
However, the lack of an association between social distance to grandmothers and grade point average does not support the grandmother hypothesis. These small sets of effects are suggestive at best. They are, however, consistent with the evidence of the benefit to academic and work adjustment that teenagers gain from extended kinship support, including grandparents (see Kenny et al. 2003; Pallock \& Lamborn 2006).

6.1.3. Behavioral and emotional adjustment. Using data from 8,752 families in the Avon Longitudinal Study of Parents and Children, Fergusson et al. (2008) explored whether childcare provided by grandparents was associated with emotional/behavioral problems in their grandchild at four years of age. The grandchild's behavior was assessed using the Strengths and Difficulties Questionnaire (SDQ) and was completed by the parent. Grandchildren who received childcare from their grandparents at 8 , 15, and 24 months of age, compared with those who received no grandparental care, were $28 \%$ more likely to score high on the hyperactivity subscale of the SDQ. This effect remained after adjustment for a range of demographic and family factors. Using the SDQ scores as continuous variables, Fergusson et al. (2008) found that compared with grandchildren with no grandparental involvement, those who received grandparental care at all three time points had slightly elevated levels of all behaviors with effect sizes "ranging from $d=.01$ (prosocial behaviour) to $d=.17$ (hyperactivity)" (p. 165). Clearly, this study highlights the fact that grandparental care is not a panacea, and future studies are needed to examine the stability of this pattern and to better understand the 
processes contributing it. Moreover, if different types of grandparents are inclined to invest differently in their grandchildren, examining all grandparents together may be masking the range of influences grandparental care can have on grandchild development.

\section{Grandparental investment in industrialized societies: Any evidence for beneficial effects under conditions of duress?}

Ordinarily, in intact families, the father is the prime candidate to provide support for the mother, followed by the maternal grandmother (Hyun et al. 2002; Levitt et al. 1986). However, it is under conditions of duress, when fathers are less likely to be available, that grandparents provide the most valuable help to their children and grandchildren (e.g., Botcheva \& Feldman 2004; Kellam et al. 1977; Leonetti et al. 2005; Oyserman et al. 1993). It is not surprising therefore that much of the research on effects of grandparental investment in industrialized societies has focused on the high-risk contexts of adolescent parenthood (e.g., Burton 1990), parental incarceration (e.g., Poehlmann 2005), children born prematurely or of low birth weight (e.g., Gordon et al. 2004), children with a disability (e.g., Mitchell 2007), and drug use and abuse by parents and children (e.g., Burton 1992; Robbins et al. 2006). In many of these high-risk situations grandparents assume the role of primary caregiver, which puts considerable strain on them and their family, and often results in poor outcomes for the grandparent. ${ }^{7}$ Reviewing these many studies across different cultures is beyond the scope of this article. We focus instead on two high-risk conditions, teenage pregnancy and maternal depression.

\subsection{Teenage pregnancy}

For good reason, the majority of teenage pregnancy studies focus on grandmother-mother-grandchild co-residence and its consequences. Co-residence represents a highrisk situation. Generally, grandparental co-residence is more common when the teenage mother is younger (Black \& Nitz 1996), and when the grandparent is younger and has multiple responsibilities, including work and family commitments (Fuller-Thomson 2005; Moore \& Brooks-Gunn 2002). Moreover, grandparental co-residence does not necessarily ensure that children and grandchildren receive higher levels of care (Lee et al. 2005).

The most common source of support for teenage mothers is their own mothers, the soon-to-be - if not already grandmothers (Tolson \& Wilson 1990). Support from grandparents, particularly grandmothers, can have a beneficial effect on young teenage mothers and their children's adjustment and development (e.g., Apfel \& Seitz 1991; Pope et al. 1993; Stevens 1988). On the other hand, evidence also shows a negative influence of grandparental co-residence on teenage mothers and their children (e.g., Chase-Lansdale et al. 1994; Schölmerich et al. 2005). These negative effects seem to arise especially where the grandparentgrandchild co-residence (a) occurs under conditions of poor family cohesion (Kalil et al. 1998), (b) is over an extended period of time (Black \& Nitz 1996), (c) occurs when the mother is older (Black et al. 2002), or (d) occurs when there are high levels of grandparental care that may be considered intrusive or may limit the development of autonomy (Spieker \& Bensley 1994; Tomlin 1998). Taken together, these findings suggest that it is most likely that grandmothers have a beneficial effect when they help rather than completely take over the mother's role or take no part in the grandchild's life (Tinsley \& Parke 1984).

\subsection{Maternal depression}

By 2020, depression is predicted to be second only to ischemic heart disease as the worldwide leading cause of years of life lost from premature death and or disability (Murray \& Lopez 1997). From the grandchild's point of view, adversities early in life - for instance, the disruption of kinship networks, and the concomitant loss of social support and material security - contribute to an increased risk of developing depression (Korkeila et al. 2005; Strassman \& Dunbar 1999). Maternal depression represents such a disruption of the family network. Individuals whose mother is depressed are at a higher risk of developing depression themselves (e.g., Weissman et al. 2006). Can grandparents in general and a high-quality relationship with one of their grandparents in particular attenuate this risk?

Conducting (in January 2008) the same literature search structure as detailed previously, but using the additional key word "depression," produced 63 studies. We found merely one study that examined the degree to which cohesion in grandchild-grandparent relationships buffered grandchildren against depressive symptoms. Silverstein and Ruiz (2006) analyzed data from 2,280 grandchildren and their mothers who participated in two waves of a nationally representative U.S. sample. Parents (at $t_{1}$ ) and children (at $t_{2}$, when they were 18-34 years old) reported the frequency with which they had experienced depressive symptoms in the past week. Cohesion in the grandchildgrandparent relationship was measured in terms of children's responses to questions regarding emotional closeness, frequency of contact, and ability to confide (when they were 10-23 years old). Children were divided into three groups, with weak, moderate, and strong cohesion. After controlling for numerous variables, such as parental income and marital history, Silverstein and Ruiz found that maternal depression ( $a t_{1}$ ) was "transmitted to grandchildren with weak and moderately strong ties to grandparents [adjusted $r^{2}=0.017$ and 0.015 , respectively], but not to those with the strongest ties [adjusted $\left.r^{2}=0.001\right]$ " (Silverstein \& Ruiz 2006, p. 608).

This longitudinal study suggests that strong ties with grandparents can help to attenuate the risk of intergenerational transfer of maternal depression. This possibility raises the question of how robust this buffering effect is across other disruptions of the family network due to a parent suffering from psychiatric or psychological disorders (e.g., addiction), parental separation, or death. A recent study by Attar-Schwartz et al. (2009), which came to our attention after our literature search had been concluded, showed that a buffering effect could be particularly valuable when the structure of the nuclear family is changed. In a representative sample of 1,515 adolescents from England and Wales, the authors examined the association between degree of grandparental involvement (from the "closest grandparent") and adolescents' behavioral and emotional adjustment as a function of three family structures: two-parent biological families, lone-parent families, 
and families with one step-parent. A higher degree of grandparental involvement, which did not differ across family structures, was strongly associated with reduced adjustment problems among adolescents from loneparent and step-parent families. Parallel beneficial effects, although mostly in the same direction, were not significant for two-parent biological families. Taken together, these observations suggest that the support of emotionally involved grandparents can be an important protective resource for their grandchildren.

\section{Public health and policy implications of grandparental investment}

Our interdisciplinary literature search showed a striking paucity of studies investigating contemporary, low-risk populations in industrialized societies. The evidence, although limited and not without warning signs, does suggest that in industrialized societies grandparents can promote the growth and development of their grandchildren. The challenge for policy makers is to create family environments and institutions where the potential grandparental resources can be harnessed. However, to this end, researchers must address the palpable gaps in the literature. Beyond the admittedly trite call for more research, we now highlight issues that we consider crucial in a future research program on grandparental investment: Specifically, we discuss the role of grandparents as public health targets, examine short- and long-term reciprocal health benefits that grandparental investments may have for the donors, and conclude by discussing the link between fertility and grandparental investment in European societies.

\subsection{Grandparents as public health targets: Future research}

Equipped with expertise and the motivation to share it, grandmothers in non-Western and indigenous societies have been targets of public health promotion to great effect: increasing birth weight in Australia (d'Espaignet et al. 2003), improving breast-feeding practices in Cambodia (Crookston et al. 2007), or improving nutritional knowledge in young mothers (Aubel 2005). Grandparents also function as health educators in industrialized societies (Watson et al. 2005), and it has been proposed that interventions target the resources grandparents bring to families (see Denham \& Smith 1989). Our empirical review suggests that the socio-emotional support grandparents provide is key to understanding how grandparental investment may influence grandchildren in industrialized societies. Evolutionary theory suggests maternal grandparents are most inclined to help, especially the maternal grandmother, and demographic data suggest that she is most likely available. Hence, one policy approach informed from an evolutionary framework is to target phenomena where the mother's family is likely to have an influence and that are influenced by socio-emotional support. Next, we illustrate two such phenomena.

8.1.1. Breast-feeding. The quintessential form of parental investment - breast-feeding - is recognized as providing health benefits for children in both developed and developing nations (e.g., American Academy of Pediatrics 1997; Hoddinott et al. 2008; Victora \& WHO Collaborative
Study Team 2000; WHO/UNICEF 1990). Female relatives, especially grandmothers, influence the duration and exclusivity of breast-feeding through their childcare, babysitting, support, encouragement, experience, and the transmission of cultural values (Bentley et al. 1999; Ekström et al. 2003; Ingram et al. 2002). For these reasons, researchers have called for grandmothers to be targeted as potential support people (Banks 2003; Bentley et al. 2003; Black et al. 2001; Ingram et al. 2003; Masvie 2006).

This support, however, is not uniformly positive. For example, sometimes the grandmotherly advice creates conflict, as it can be inconsistent with current best health practices (Bentley et al. 2003; Duong et al. 2005). Once well informed, however, grandparents appear to be willing to change and integrate new health information with their pre-existing knowledge (Aubel 2005; Aubel et al. 2004). Ingram et al. (2003), for instance, aimed to help grandmothers from Pakistani, Bangladeshi, and Indian communities living in Bristol, England, to support exclusive breast-feeding by their daughters and daughters-in-law. The focus on this group was because of low exclusive breast-feeding rates (Thomas \& Avery 1997), and the intervention was shown both to improve the grandmother's knowledge about breast-feeding and to increase the mother's breast-feeding rate.

8.1.2. Postpartum depression. According to one adaptationist hypothesis for postpartum depression (PPD), which afflicts 10 to 15 percent of new mothers, PPD may have evolved as a strategic response to a lack of social support (Hagen 1999). Specifically, problems with the pregnancy, delivery, or infant, and perceived lack of the social support necessary to raise a baby appear to be closely associated with PPD (Beck 2001; O'Hara \& Swain 1996). Mothers suffering from PPD reduce their investment in their new baby (see Hagen [1999] for a review of the evidence). Few interventions, except for the intensive postpartum support from a health professional, seem to reduce the symptoms of PPD (Dennis 2005). Although there are indications that grandparents could provide the social support necessary to reduce the symptoms of postpartum depression (Shanok \& Miller 2007 ), to the best of our knowledge, there are no detailed analyses of this potentially beneficial mental health impact.

Let us conclude with a cautionary note. The presence of grandparents is not a fail-safe remedy. There is likely to be no simple relationship between grandparental presence and the incidence or outcome of breast-feeding or postpartum depression. The quality of the grandparentparent relationship is likely to be an important moderator. If the grandparent-mother relationship is confrontational or demanding, a grandparent's involvement may actually exacerbate a mother's depression (Hess et al. 2002). This suggests that a research program on grandparental investment stands to benefit from exploring the determinants of grandparental investment, such as type of grandparent, identified within an evolutionary framework (see Table 1), and values, norms, and satisfaction with grandparental roles, identified within a sociological framework.

\subsection{Grandparental investment: A one-way street?}

In our aging societies, it is increasingly important also to consider the benefits that grandchildren can provide for 
grandparents (beyond increased fitness), especially grandparental health, rather than focusing exclusively on the reversed relationship. What could such benefits be? One obvious benefit that follows immediately from the view that grandparental investment is an intertemporal exchange between self-interested grandparents, parents, and grandchildren is that grandparents who have cared for their grandchildren may be more likely to receive support from them or their parents in times of need (Friedman et al. 2008; Stack 1975). Such help matters. Esbensen et al. (2007) showed that direct support from grandchildren could improve a grandparent's health during times of severe physical illness. Relatedly, in a study of 2,200 older Japanese people, Okabayashi et al. (2004) found that, in those individuals who did not have a spouse $(n=677)$, social support from children and grandchildren was associated with more positive well-being, fewer depressive symptoms, and reduced cognitive impairment.

Another short-term benefit of grandparental investment is that grandchildren provide company. Individuals who live in the company of more kin generally have improved health and a higher probability of surviving crisis situations (Sugiyama 2004). Consistent with such findings, in their study of 442 grandparents from the Longitudinal Study of Generations, Drew and Silverstein (2007) observed that grandparents who lost contact with at least one of their grandchildren exhibited a steadily increasing incidence of depressive symptoms between 55 and 80 years of age. In contrast, in grandparents who experienced no loss of contact with their grandchildren, depressive symptomatology remained stable. This effect was robust to the influence of gender, education, marital status, number of children, and linear change in health.

Although these studies observed ways in which grandchildren can benefit grandparents, they did not condition these effects on grandparents' past investment behavior. The two studies of which we are aware that examine the future effects of grandparental good deeds are by Fujiwara and Lee (2008) and Hughes et al. (2007). Fujiwara and Lee found that grandfathers and fathers (not grandmothers and mothers) who provided moderate amounts of informal or financial support had a lower risk of developing major depression three years later. This association was robust to the influence of additional factors associated with the risk of major depression, including major depression at baseline. Using data from the Health and Retirement Study, Hughes et al. found that grandmothers who babysat for their grandchildren showed higher levels of self-rated health and were more likely to exercise two years later compared with grandmothers who provided no care.

To conclude, the available evidence suggests that altruistic acts towards others (e.g., grandchildren) can have beneficial consequences for the altruist's (e.g., grandparent's) own physical and mental health (McClellan et al. 1993), including a reduced risk of morbidity and mortality (Brown et al. 2005; Brown et al. 2003). However, as before, let us not lose sight of the boundary conditions of grandparental altruism. When the demands of helping become too much, the impact on health can be negative (Goh 2009; Lee et al. 2003; Post 2005). That is, just as grandparents who lose contact with their grandchildren experience deterioration in their physical and emotional health (Drew \& Silverstein 2007; Drew \& Smith 2002), so too do those who assume primary caregiver responsibilities (Heywood 1999; Ross \& Aday 2006).

These findings suggest that moderation, as with many things in life, in the amount of care grandparents give is likely to optimize grandparental well-being. Figure 1 stylizes this hypothesized nonlinear relationship between grandchild care and grandparental well-being. Myriad factors, such as the availability of resources, gender, and life transitions (e.g., retirement), will affect the nonlinear relationship depicted in Figure 1. Perhaps one of the most important moderating factors is the degree to which grandparents are overstrained by the simultaneous demands of caring for both their ailing parents and grandchildren (Cooney \& An 2006; Grundy \& Henretta 2006). Interestingly, the same nonlinear relationship may be true for the grandchild's well-being: Very low and very high levels of grandparental involvement can have a negative impact on the development of grandchildren (Lavers \& Sonuga-Barke 1997).

\subsection{Contemporary grandparental investments and fertility: How and why are they related?}

Let us return to one key evolutionary account of grandparental altruism. Could grandparents in industrialized societies boost their own fitness by investing resources in their children's and grandchildren's survival and reproduction? We do not know of any study into this possibility, and this lack is not surprising given the demographic changes in industrialized societies (sect. 5). There are, however, separate lines of research documenting fertility trends and levels of grandparental childcare across countries. Connecting these two lines reveals an interesting picture for Europe. Using data from the 2004 Survey of Health, Ageing and Retirement in Europe (SHARE), Hank and Buber (2009) have reported the percentage of grandparents who provided any care (over the last 12 months), and who provided regular care (i.e., almost weekly or more often over the last 12 months) across ten European countries. The trends for both kinds of care are reversed. The prevalence of any care is highest among Danish, Dutch, French, and Swedish grandparents

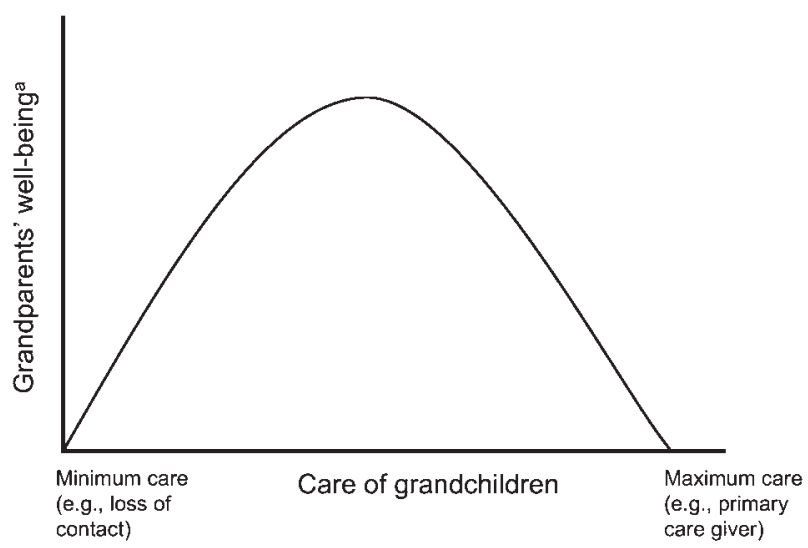

Figure 1. A schematic representation of the hypothesized nonlinear relationship between the care grandparents take of their grandchildren and the grandparents' well-being.

Note. ${ }^{\text {a }}$ Well-being encompasses various positive emotions such as satisfaction and contentment, and positive activities, such as spending time in company. 
and lowest among Italian and Spanish grandparents. In terms of regular care, however, Greek, Italian, and Spanish grandparents score highest, whereas Danish, French, and Swedish grandparents contribute least. When one correlates the likelihood of regular care with total fertility rates in the ten European countries studied by Hank and Buber, one finds a strongly negative correlation (Spearman rank correlation $=-.88, p=0.008$; we calculated this correlation using estimated fertility rates for 2009; Central Intelligence Agency 2009). That is, surprisingly, high levels of investment by grandparents are associated with low fertility rates.

Why is that? Among the key variables contributing to this relationship are likely to be women's participation rate in the labor market and the availability of institutional care (Billari \& Kohler 2004). In Europe, countries that value gender equality are likely to provide state-subsidized day care and other institutional support for working women, thereby reconciling the conflict between motherhood and work. In contrast, countries such as Greece, Italy, and Spain have relatively little state-financed day care, and women are more likely to stay at home after they become mothers. Indeed, Italy and Spain have markedly lower labor force participation of women than, for example, Denmark, France, and Sweden. Because of the lower female participation rate, there is, as Hank and Buber (2009) suggest, less demand for grandparents to help out in Italy and Spain, relative to, say, Denmark. If, however, a Mediterranean mother decides to have children and continue to work, she has to rely on grandparents' support on a regular basis (for U.S. data, see Presser 1989). Consequently, parents of a working mother might have been key to their daughters' decision to have children. For these grandparents, grandparental care and reproductive success might indeed be closely related. More generally, this initial and speculative analysis illustrates that studying the interplay of evolutionary (e.g., fertility), economic (e.g., women's participation rate in the labor market), and institutional variables (e.g., availability of institutional care) promises to yield new insights into the impact of grandparental investment in industrialized societies.

\section{Grandparental investment: A research program for the future}

From babysitting to support when the kids set fire to the carpet, having grandparents around can be invaluable. The extent to which grandparents' presence and support matter and can be explained has piqued the interest of various disciplines. Approaches, however, have often remained within the boundaries of one discipline. In industrialized societies, however, grandparental transfers cannot be understood without analyzing how institutions (e.g., social welfare regimes, inheritance laws) shape them. Analyses of institutions, in turn, need also to consider how proximate mechanisms, such as values, norms, and the empathy mechanism, may protect grandparental care from being crowded out. In addition, determinants derived from an evolutionary framework (Table 1), in combination with interindividual differences in values, can be key in predicting and explaining systematic variability among grandparents. Multiple disciplinary theories and hypotheses are in place - one task for the future is to get serious about integrating them into a larger framework. Based on this, a series of old and new questions await answers. As revealed by our review, we know relatively little about the hypothesis we have explored here, namely, that the beneficial effects of grandparental investments in industrialized societies may reside in "softer" dimensions, such as the grandchildren's cognitive and verbal ability, mental health, and well-being. Yet, there is suggestive evidence that the help of emotionally close grandparents can be an important asset under circumstances of duress. This potential, however, deserves to be studied across the manifold circumstances of duress in which social support matters, some of which are listed here. Moreover, to the extent that grandparents are targeted as public health assets, we need to better understand the boundary conditions of the beneficial impact and the counterproductive effects, and examine the various costs and benefits that grandparents reap from their investment. We hope that our review is a first step toward a more encompassing research program on grandparental investment.

\section{ACKNOWLEDGMENTS}

Our thanks go to James Chisholm, Edward Hagen, Sarah Hrdy, Debra Judge, Barbara Marti, Kathy Sanders, Rebecca Sear, Virgina Vitzthum, and five anonymous referees for many constructive comments. We also thank Laura Wiles for editing the manuscript, and Patrick Riepl and Ursina Pieth for their help in conducting the literature searches.

This work is part of the National Centre of Competence in Research (NCCR) Swiss Etiological Study of Adjustment and Mental Health (SESAM). The Swiss National Science Foundation (SNF) (project no. 51A240-104890), the University of Basel, the F. Hoffmann-La Roche Corp., and the Freie Akademische Gesellschaft provided core support for the NCCR SESAM. This publication is the work of the authors, David A. Coall and Ralph Hertwig, who serve as guarantors for the article.

\section{NOTES}

1. Fossil records suggest that the extended postreproductive lifespan in women and the long period of childhood development appeared at roughly the same time in human ancestry (Bogin 1997; Bogin \& Smith 1996). This is consistent with the proposition that both of these unique features of the human life history - most likely linked by a general increase in longevity - evolved together as a self-reinforcing unit (e.g., Carey \& Judge 2001). Evidence based on extinct hominid species suggests that the uniquely long human lifespan is not a recent development (possibly due to modern medicine; Weiss 1981). Hammer and Foley (1996) calculated estimates for hominid longevity using 13 anthropoid primate subfamilies and five extant ape species. The longevity estimates for the Australopithecines (2-4 million years ago) as a whole (45-53 years) overlap with those of modern chimpanzees ( $47-50$ years). By 2.4 to 1.6 million years ago, Homo habilis was estimated to have a longevity of 52-56 years, which exceeds age at menopause in contemporary human populations. Longevity estimates increased dramatically in Homo erectus (60-63 years), providing some 15-18 years of postreproductive lifespan (Judge \& Carey 2000). The estimates continued to rise for Homo sapiens (66-72 years), and reached 78 years when estimates were based on the brain sizes of anthropoid primates, which are compatible with extant hunter and gatherer populations (Blurton Jones et al. 2002; Gurven \& Kaplan 2007; see lower estimates by Caspari \& Lee 2004; McHenry 1994).

2. We refer to both hypotheses to represent two lines of work that attempt to understand the evolution of menopause and 
longevity in humans. Each of these perspectives, however, consists of several different hypotheses. For example, the goodmother hypothesis (Alexander 1974; Sherman 1998) has several similar variants including the stopping early hypothesis (Hawkes et al. 1998; Williams 1957), the prudent-mother hypothesis (Hrdy 1999), and the altricial lifespan hypothesis (Peccei $1995 ; 2001 b)$. The same may also be said of the grandmother hypothesis (see Peccei 2001a).

3. The beneficial influence of mothers on the survival of their children is universal (Sear \& Mace 2008) and may have been the initial advantage for a post-reproductive lifespan in humans (e.g., Alexander 1974; Fedigan \& Pavelka 2001; Hrdy 1981; Lancaster \& King 1985; Lancaster \& Lancaster 1983; Madrigal \& Melendez-Obando 2008; Packer et al. 1998; Pavard et al. 2007; Peccei 1995; 2001b; Penn \& Smith 2007; Sherman 1998). In addition, there are also integrative hypotheses regarding the evolution of menopause, but they have not been thoroughly tested yet (e.g., Cant \& Johnstone 2008).

4. This pattern of grandparental investment has been shown across a wide range of grandparent-grandchild relationship variables, including care during childhood, emotional closeness, relationship closeness, financial support, and contact (see Bishop et al. 2009; Bridges et al. 2007; Chrastil et al. 2006; Dubas 2001; Eisenberg 1988; Euler \& Weitzel 1996; Hoffman 1980; Kahana \& Kahana 1970; Laham et al. 2005; Mills et al. 2001; Monserud 2008; Pashos 2000; Pollet et al. 2009; Scholl Perry 1996; Smith 1991; Steinbach \& Henke 1998; Uhlenberg \& Hammill 1998).

5. The downward flow of resources from older to younger members of a society or family appears to be a consistent pattern found in both contemporary traditional and Western societies (e.g., Attias-Donfut et al. 2005; Bengtson 2001; Caputo 1999; Essock-Vitale \& McGuire 1985; Hogan et al. 1993; Kaplan 1994; Kohli 1999; Kotlikoff \& Summers 1981; Lee 1997; Schröder-Butterfill 2004; Streib 1958; Szinovacz 1998a; Turke 1988).

6. The other nine publications are: Cox 2000; Crosnoe \& Elder 2002; Healy 2000; Hurme 2006; Lavers \& Sonuga-Barke 1997; Lin 2003; Schultz 1980; Thompson et al. 1989; Yong 2006.

7. Although many grandparents feel that it is their responsibility to help raise their grandchildren when their biological parents are not available, being a custodial grandparent impacts negatively on grandparents' physical and emotional health, their economic hardship, and their own social networks (Burton 1992; Edwards 2006; Fuller-Thomson 2005; Fuller-Thomson \& Minkler 2000; Hayslip \& Kaminski 2005; Heywood 1999; Joslin \& Harrison 2002; Lee et al. 2005; Oburu 2005; Oburu \& Palmerus 2005; Ross \& Aday 2006).

\section{Open Peer Commentary}

\section{Grandparental investment and the epiphenomenon of menopause in recent human history}

\section{doi:10.1017/S0140525X09991579}

\section{Douglas C. Broadfield}

Department of Anthropology, Florida Atlantic University, Boca Raton, FL 33431.

broadfie@fau.edu

\footnotetext{
Abstract: The effects of grandparental investment in relatives are apparent in human groups, suggesting that a postreproductive period in humans is selective. Although investment of relatives in kin produces obvious benefits for kin groups, selection for a postreproductive period in humans is not supported by evidence from chimpanzees. Instead,
}

grandparental investment is likely a recent phenomenon of longevity, rather than an evolved feature.

Grandparenting has clear advantages in modern societies, producing benefits that affect social outcomes. The effect is such that Coall \& Hertwig $(\mathrm{C} \& \mathrm{H})$ propose a host of potential benefits, and some consequences, to be studied as a result of the influence of grandparenting. However, despite the sociological and economic effects of grandparenting studied today, there is little information regarding the evolution of grandparenting. Williams' (1957) assertion that early termination of fertility would be adaptive when maternal care is important to offspring survival, proposes that reproductive senescence is adaptive by enhancing the inclusive fitness of grandparents. While this premise has been modified several times in the past fifty years, all of the current hypotheses surrounding the evolution of grandparenting make the assumption that any feature, especially that which brings benefit to the family group, must be adaptive.

If it is adaptive, then menopause is a human autapomorphy. While it has been suggested that chimpanzees (Atsalis \& Videan 2009; Jones et al. 2007) and gorillas (Atsalis \& Margulis 2008) go through menopause if they live long enough, other studies (Cloutier et al. 2009; Emery Thompson et al. 2007) suggest that menopause as it is applied to humans does not exist in chimpanzees. Chimpanzees fail to go through operational menopause, the cessation of menstrual cycling, as humans do. Indeed, chimpanzees have been recorded to have offspring well past the age of forty (Cloutier et al. 2009). Although chimpanzee females may not spend their lives in the same group as offspring, most certainly spend years as grandmothers before daughters emigrate. However, there is no evidence that the grandmother's fecundity is reduced by the presence of their daughter's offspring. If one assumed that menopause should be adaptive and lead to the evolution of grandparenting, then it would seem likely that the trait should have evolved in our closest living relatives during their five million year history. However, this has not been the case, suggesting that menopause is a uniquely human character.

Adaptation assumes that a feature enhances fitness, and has the opportunity to be under selective pressure. While it has been argued that menopause is adaptive by protecting the individual from survival risks to herself or her potential offspring (Kuhle 2007; Peccei 2001a), all of the hypotheses overlook the fact that menopause and life expectancy into postreproductive years are recent phenomena of human evolutionary history. Prior to 1900 , there is little evidence to support menopause as a universal phenomenon in humans due to low life expectancy (see Christensen et al. 2009). This suggests that prior to the 20th century menopause was not under selective pressure, as those individuals who lived into their post-menopausal years were few. As a result, the effect of living beyond reproductive years is a phenomenon that could have only recently come under selective pressure. However, it is unlikely that characters expressed in postreproductive years could be under selective pressure.

Current life expectancy projections for Europe and North America (Christensen et al. 2009) suggests that humans can expect that almost half of their lives will be spent in postreproductive years. This could suggest that any benefit produced through a grandmother effect would be erased by the demands of offspring to care for grandparents. If maximum life expectancy were realized in humans, then the evolutionary costs could quickly outweigh the gains. However, in assuming that there is selective pressure on a postreproductive character, one has to assume that the character can be selected at all.

In the wild, few female chimpanzees survive past fifty (Hill et al. 2001), yet many become grandparents during their life. It is suggested that a similar life history was the norm throughout human evolution until recently (Caspari \& Lee 2004). Indeed, it is only recently that both humans and chimpanzees have 
been found to live into either their postreproductive years (humans) or their pseudo-postreproductive years (chimpanzees). In the case of chimpanzees there is no current evidence of a cessation of menstrual cycling or a complete loss of fertility (Cloutier et al. 2009; Emery Thompson et al. 2007), whereas in humans there is clear evidence that humans cease cycling after approximately fifty years of age. This disparity makes chimpanzees poor models for the evolutionary development of menopause.

Instead, menopause must be viewed in light of human evolutionary history alone. Although it can be argued that a postreproductive period enhances fitness by reducing the risks of reproduction on the mother and the offspring, it is unlikely that the postreproductive period can be viewed as adaptive for the function of grandmothering. Ellison (2001) suggests that menopause could be an exapted feature of the depletion of a female's supply of oocytes. This implies, however, that oocyte depletion is adaptive. Although the quality of oocytes decreases in a female's lifetime, there is little to suggest that the feature should be selected. Again, there is little evidence suggesting a decline in the quality of oocytes in chimpanzees with age. If chimpanzees evolved the ability to continue to produce viable offspring to the end of their lives, then it would stand to reason that the same feature should be present in humans. That the feature is lacking in humans may not be a reflection of an adaptation to preserve oocyte quality, but instead could suggests that humans possess the feature as an epiphenomenon (Bogin \& Smith 1996).

It is apparent that humans have benefited from relatives living into their postreproductive years. However, these benefits should not be viewed as adapted or selected, since their presence in human populations is recent. If menopause evolved to reduce the risks to the mother and offspring, then it should have evolved to begin at age 35, an age when the likelihood of risks to mother and offspring dramatically increase (Morris \& Alberman 2009). In addition, there is little evidence to support the claim that there can be selection for a postreproductive period that would lead to grandparenting. More likely, grandparenting is a happy byproduct of other human characteristics, making it at best an epiphenomenon.

\section{Integrating evolutionary and social science approaches to the family}

doi:10.1017/S0140525X09991580

\section{Donald Cox}

Department of Economics, Boston College, Chestnut Hill, MA 02467. donald.cox@bc.edu

http://www2.bc.edu/ coxdo/

Abstract: Recent work on the evolution of utility has brought the blunt instrument of kin selection closer to the cluttered scalpel kit of social science. The concept of diminishing marginal utility can help streamline the latter. Reconciling ultimate causes with proximate inclinations, however, will be easier for the case of assistance from grandparents than assistance to them.

The surest way to better understand grandparenting - or most anything else about families - is to heed Coall \& Hertwig's (C\&H's) call for interdisciplinary mutualism. They are exactly right: Pitting evolutionary and social science as contenders for center stage gets us nowhere. Progress will come from recognizing that the two approaches emanate from distinct corners of a single - and woefully incomplete - puzzle.

Advances will require continuing efforts to connect distant dots - ultimate and proximate forces - to plumb the interplay between, say, kin selection's primal tug and the exigencies of hedonics and budgets, played out in the context of real-world culture and institutions.
Fortunately, more work exists along these lines than the few harbingers of hope $\mathrm{C} \& \mathrm{H}$ mention. What they aptly call a "motivational engine" was added to evolutionary models more than a decade ago, when economists replaced the standard maximand of reproductive success with something just as fundamental but more immediate individual well-being (i.e., "utility"), which includes feelings for other family members (e.g., Alger \& Weibull, forthcoming; Bergstrom 1997). This refinement retains the essence of kin selection (i.e., caring about one's genetic legacy), but fleshes out the details about how much help is provided and when. Help is subject to diminishing marginal utility: the better off the recipient becomes, the smaller the impact of additional help; the worse the predicament, the more help matters. Diminishing marginal utility is a simple but powerful idea.

For example, grandparents and would-be grandparents have been found to be most likely to assist their adult children with housing down payments when the children: (a) wanted to have a child of their own but (b) were concerned that their present living quarters were too small to accommodate one (Cox \& Stark 2005). The first reason is arguably tied up with the ultimate motive of fitness; the second, with the proximate motive of marginal utility: All else equal, the smaller the adult child's living space, the more grandparental assistance matters.

Diminishing marginal utility can explain C\&H's finding that grandparenting apparently confers less widespread or pronounced benefits for "low risk" families compared to their "high risk" counterparts, where teenage pregnancy or maternal depression occur. Large-scale help would be less consequential, and hence less prevalent, for grandchildren who are already well off. Evolution determines the capacity for altruism; but proximate factors like diminishing marginal utility determine whether and how this capacity is expressed.

Diminishing marginal utility can also help reconcile seemingly disparate motives for kin assistance. Consider altruism and exchange, which $\mathrm{C} \& \mathrm{H}$ - and just about every other researcher cast as competing motives. The logic of helping behavior can be simplified and enriched by considering how the two might coexist and when one or the other might dominate.

For instance, imagine that Aaron and Ben are colleagues in a firm. At a company picnic by a lake, Aaron hears Ben's cries for help, realizes he is drowning, and prepares to risk his life to save him. Fast-forward to a time long after Ben's rescue. The urgency of altruistic transfers has fallen; Ben is no longer someone at death's door - he is now just someone who works down the hall. Aaron might still help Ben, but only quid pro quo: the same people, but different circumstances and hence different motives for helping.

This portmanteau approach has the counterintuitive implication that the worse off recipients are, the more likely the introduction of public transfers will crowd out private giving. Return to our example and suppose a town lifeguard were to save Ben, thereby letting altruist Aaron off the hook. To cast things in money terms, imagine that social insurance aids impoverished Ben, thereby supplanting Aaron's help. But if Ben were already well off enough that the relevant motive were exchange, increases in Ben's income would only serve to strengthen his bargaining position with Aaron, thereby generating "crowding in."

The contrasting income effects can explain why evidence for crowding out is scarce for Europe and the United States but not for developing countries, where private assistance matters more for addressing basic needs (Cox \& Fafchamps 2008).

How does a proximate motive like altruism mesh with the ultimate influence of kin selection? If Aaron were Ben's grandfather, it is easy to see how they would dovetail. And generational altruism in the opposite direction - say, care that adult children provide to their parents - could be reconciled by appealing to the fitness benefits of grandparenting, as in "The sooner I can get Mom back on her feet, the more she can do for my kids."

But what about instances of elder care with no apparent fitness advantage, such as an adult child who, amid demands of work and 
childrearing, makes time for her frail and indigent mother? Some upstream transfers appear to fit this description (e.g., Perozek 1998; Sloan et al. 1997), yet they pose a difficulty for kin selection that has attracted surprisingly little notice in the evolutionary literature. A gene that impelled the provision of such care would eventually be out-competed by fitness-enhancing ones. The same goes for the evolution of cultural practices (remember the Shakers?).

What about empathy, a motive deeply ingrained yet focused on today's cues, rather than tomorrow's fitness? Surely the care described above would qualify as such - the key question is how it could prevail in an evolutionary environment. If, in the authors" words, "empathy-based acts are allocated in accordance with kin and reciprocal altruism theory" (target article, sect. 4.3, para. 5), they had better not be allocated too strictly or we are back to square one. One could speculate that unrequited elder care is somehow tied up with the forces of group selection group-beneficial norms against preventable suffering, say - and provided out of fear of altruistic punishment. Or, that it is a byproduct of deep-rooted inclinations to reciprocate, or perhaps a signal of willingness to cooperate with non-kin. Finding the evolutionary basis for this expression of empathy appears to require connecting dots that for the moment appear exceedingly distant. But it is likely a feasible task, and certainly one that would be worthwhile.

\section{ACKNOWLEDGMENTS}

I thank Tracy Vietze and Ingela Alger for comments. Support from the National Institutes on Child Health and Human Development (R01-HD045637) is gratefully acknowledged.

\section{Is contemporary grandparental care an evolutionary mismatch?}

\section{doi:10.1017/S0140525X09991592}

\section{Harald A. Euler}

Institute of Psychology, Department of Economics, University of Kassel, 34127 Kassel, Germany.

euler@uni-kassel.de

http://www.psychologie.uni-kassel.de/indexpers.htm

\begin{abstract}
In order to evaluate the impact of contemporary grandparenting, the view should not be restricted to developmental achievement effects in grandchildren. Both child happiness and grandparent happiness are high-ranking goals with implications for public policy. The beneficial impact of grandparenting for risk families appears unequivocal, and modern life still encounters all kinds of unpredictable risks. Contemporary grandparental care is no evolutionary mismatch.
\end{abstract}

Up until a few decades ago, grandparenting was no salient topic in the sciences of human behavior. As Coall \& Hertwig $(\mathrm{C} \& \mathrm{H})$ have shown, it has become a topic of study on which researchers have weighed in from their respective disciplines. Grandparenting is now studied by sociologists, biologists, anthropologists, and evolutionary psychologists. The academic disciples differ in their contributions. Sociologists tend to interpret data a-theoretically. Their resistance to biological accounts of human behavior, however, frequently prevents a cut of nature at its joints: the distinctions between grandparent sexes, or lineage (parent sexes), or both, are frequently omitted in social science research (e.g., Patrick \& Goedereis 2009). Both variables, however, are pervasively important. Anthropologists, inspired by the Grandmother Hypothesis, inspect hard reproductive data such as number of offspring or grandchild mortality. Psychologists usually present soft data, such as rated emotional closeness or grandparental solicitude. $\mathrm{C} \& \mathrm{H}$ have done a commendable job in bringing the various approaches together and highlighting their respective contributions fairly. Their review is, in my opinion, one of the very best about grandparenting.

I take the liberty to comment on the results from Tinsley and Parke (1987). C\&H interpret these results as suggestive of the beneficial effects of grandparenting on the mental development of grandchildren. C\&H prudently say that the data suggest an association which is not necessarily causal. A genetic confound might be excluded by the observation that more significant associations (namely, 7) between grandparent-infant interaction patterns and infant development scores were found for grandmother-infant dyads than for grandfather-infant dyads (namely, 2). But the study does not indicate the extent to which these associations are grandparent-driven, as $\mathrm{C} \& \mathrm{H}$ assume, or grandchild-driven.

More important, however, is a finding by Tinsley and Parke (1987) which I consider highly relevant for the question of whether grandparenting does any good in modern Western countries. Cui bono? Grandparental care might not only be good for grandchildren (and parents, for that matter), but also for the grandparents themselves, provided the required grandparental involvement does not exceed the grandparental resources. $\mathrm{C} \& \mathrm{H}$ acknowledge the possible benefits of grandparenting for grandparents at the end of the target article, but they might have overlooked a noteworthy finding by Tinsley and Parke: namely, that contact with the infant was rated substantially and significantly more satisfactory by grandparents than by parents, this finding applying equally to both genders of grandparent/parent. From an evolutionary perspective, this point is to be underlined. Nepotistic effort is the salient life effort during the sunset years (Alexander 1987), and it therefore comes as no surprise that its practice is perceived as highly satisfying.

An impression left by the studies reviewed by $\mathrm{C} \& \mathrm{H}$ is that the effects of grandparental care-giving count if they have an impact on the achievement scores of grandchildren. The value of grandparenting ought not to be so narrowly defined. In addition to achievement, there is also well-being, a feeling of connectedness, happiness, and life quality, for both the grandchild and the grandparent. Objective measures (e.g., survival rates, achievement scores) as well as subjective measures need to be considered for grandparents, parents, and grandchildren.

$\mathrm{C} \& \mathrm{H}$ clearly describe the consequences of the demographic transition for grandparent-grandchild relationships. The shared lifespan between grandparents and grandchildren is bound to become shorter; and increasingly there is only one grandchild. We might ask whether deprivation of contact with grandchildren might have adverse effects on the life quality of the aged. I have had grandparents in my office weeping bitterly of their biggest misfortune: that they were denied contact with their only grandchild. Legislators have become sensitive towards this problem. The recognition of grandparents' visitation and custody rights is a fairly recent trend.

Also rather recent is the increased consideration of grandparents in public child welfare (Herring 2008). Kin are increasingly used as foster parents. In the United States, the most common placement for foster care is with grandparents, usually the maternal grandmother (Scannapiego \& Hegar 2002). Legislators and welfare agencies prefer to listen to the expert advice of social scientists rather than evolutionary biologists, but the blatant disregard for biological variables may come with costs. There are asymmetries between the sexes which do not disappear by "deconstruction" or by benign neglect. If, for example, sociological studies investigate step-parent households - defined as those with either a stepmother or a stepfather - an important distinction is overlooked, namely, whether the biological parent with whom the child co-resides is the mother or the father. Mother absence is more disadvantageous to the child's development than father absence, both with respect to the investments made in children and with respect to child outcomes (Case et al. 2001), and parental investments are lower in stepmother families 
than in birth mother families (Case \& Paxson 2001). Most telling and directly relevant for grandparental care-giving are the findings by Duflo (2003), who examined the flow of pensions given in South Africa to grandparents living with their grandchildren. Disturbingly, only the pensions to maternal grandmothers were channeled on to grandchildren.

The review by $\mathrm{C} \& \mathrm{H}$ shows that grandparental investment protects children from adverse effects in certain family situations with high risks. Risky situations may, at first glance, appear to be a matter of past epochs of famines, epidemics, and recurrent wars, or seem rare cases with little importance for normal modern families. But the list of risks, which is not exhaustive, includes maternal depression, teenage pregnancy, lone-parent, and stepparent families. These risks have such a high prevalence in modern societies that there is, and always will be, a need for helping grandparents whose assistance is good for the grandchildren - and also good for the grandparents, as long as they are not the sole caretakers. Life has been risky in the past and will remain risky in the future, even if the types of risks change with time. Therefore, contemporary grandparental care is not an evolutionary mismatch, an adaptation advantageous in ancestral environments but useless in modern times, like the hedgehog's instinct to roll itself inwards when a car approaches.

\section{Grandparental altruism: Expanding the sense of cause and effect}

\section{doi:10.1017/S0140525X09991609}

\section{Edmund Fantino and Stephanie Stolarz-Fantino}

Department of Psychology, University of California-San Diego, La Jolla, CA 92093-0109.

\section{efantino@ucsd.edu}

sfantino@psy.ucsd.edu

\begin{abstract}
Grandparental altruism may be partially understood in the same way as other instances of altruism. Acts of altruism often occur in a context in which the actor has a broader sense of cause and effect than is evident in more typical behavioral interactions where cause and effect appear relatively transparent. Many believe that good deeds will ultimately produce good results.
\end{abstract}

The target article by Coall \& Hertwig $(\mathrm{C} \& \mathrm{H})$ makes a strong case for the need to integrate different perspectives on grandparental investment, as well as for the difficulty of harboring all perspectives under a single convenient tent.

An additional argument against the sufficiency of theories that rely heavily on genetic relatedness comes from reports of interspecies adoptions. For example, Izar et al. (2006) reported on the case of adoption of an infant marmoset by a group of wild capuchin monkeys. Cross-species fostering is observed more commonly in domestic animals; for example, Hersher et al. (1963) studied parameters that facilitated cross-species adoption between sheep and goats. Psychologists might point out that such behavior represents generalization from behaviors appropriate for the (adoptive) species toward conspecifics. Kuo (1930; 1938) reported that when single kittens were raised with single rats, they appeared to be attached to one another; when groups of kittens and rats were raised together, the cats and rats appeared indifferent to each other. A further argument is an extension of C\&H's point that the proximate cause for grandparental care of their children and grandchildren lies in the grandparents' capacity for empathy, a point stressed by Hrdy (2009) and also by Fantino and Stolarz-Fantino (2002) in a Behavioral and Brain Sciences commentary on Rachlin's target article "Altruism and Selfishness." In any event, the mechanism of empathy certainly need not be restricted to kin. In Fantino and Stolarz-Fantino (2002), we address the classic problem of a woman rushing into a burning building to save an unknown crying baby. We pointed out that negative reinforcement could provide an account of altruism here: The altruist avoids the indelible future memory of having done nothing to save the baby if she does not rush to help. In addition, we point out an array of reinforcement principles that can account for instances of altruistic behavior. Our experiences surely help to shape our propensity to be altruistic or not. For example, grandparents may well be excellent at caring for their grandchildren, having learned how to do so when caring for their children (and having modeled some of their own parenting behaviors upon those of their parents). Thus, a pattern of care-giving behavior has been acquired.

Of course, not all grandparents behave caringly towards their grandchildren, nor do humans and nonhumans regularly behave altruistically. In our laboratory, using one measure of altruism, the Sharing Game (an economic activity related to the Dictator Game; Fantino \& Kennelly 2009; Kennelly \& Fantino 2007), $20 \%$ of participants typically select altruistically, forsaking a payoff entirely in order to enrich an unknown other participant whom they will never meet - when given a choice such as the following:

Player One receives $\$ 10$ and Player Two receives $\$ 10$

\section{OR}

\section{Player One receives $\$ 0$ and Player Two receives $\$ 100$}

In this choice, it was clear that real money was involved and that the two players would never meet and would remain anonymous. We have in fact now done an approximation to this study three times conducted by three different experimenters; each time approximately $20 \%$ of participants (college students) chose altruistically, being willing to distribute a substantial sum to the anonymous second participant while taking nothing for themselves. Male participants were far more likely to select the altruistic option; this gender difference may reflect our published finding that female participants prefer to make egalitarian selections in the Sharing Game.

Not all instances of altruistic behavior may be accounted for by obvious instances of reinforcement, nor with consideration of genetics, relatedness, or individual experiences. A broader perspective might involve an expanded view of the self, and even the idea that individuals' behavior has important effects on their milieu, such that "what goes around comes around." For example, C\&H discuss studies of altruistic punishment (e.g., Fehr \& Gachter 2002; see also, Zizzo \& Oswald 2001). A type of altruistic punishment ripped from the headlines involves the punishment of those who talk loudly on their cell-phones in public places (as reported by Douglas Quenqua in an article in the New York Times; Quenqua 2009). The punisher may gain no benefit for himself but may improve the environment in which we all reside. The more the cell-phone abusers who are silenced, the quieter the environment for all of us. Also, it is widely believed that an environment filled with litter tends to attract more littering. Thus, if we punish littering, we may improve the environment for us all. In both of these examples, however, the relationship between the altruistic act and the benefits to the individual are extremely indirect and even tenuous. The problem is similar to one raised by $\mathrm{C} \& \mathrm{H}$ concerning how we might "bridge the gap between altruistic acts now and ultimate reproductive success benefits in the future" (sect. 4.3, p. 6). In addition to an account in terms of empathy (with which we concur), the problem may be at least partially solved in two ways. First, it is not enough to think in terms of a "self" restricted to the boundaries of the individual body. We live in an environment that has largely nurtured us (though for some for whom this has not been the case, the propensity for altruism may be low indeed). When we act in a way that benefits that physical and social environment, we can feel that we are benefiting ourselves. The benefit need not be immediate. For most of us, strict reciprocal altruism is not required. We have an expanded 
view of cause and effect. Thus, most of us believe in what might be thought of as "what goes around, comes around": Good that is done ultimately comes back to us. To assess this, we asked more than 300 undergraduates here at UCSD to what extent they agreed with the statement, "What goes around, comes around." Eighty-six percent of students were in agreement (only $8 \%$ disagreed; the others were neutral). Thus, the gap between altruism and its payoff may simply reside in the belief that good deeds are ultimately rewarded. This type of belief may help buttress the other causes that are so eloquently brought to bear by $\mathrm{C} \& \mathrm{H}$ to account for grandparental investment and altruism.

\section{Intergenerational conflict over grandparental investment}

\author{
doi:10.1017/S0140525X09991610
}

Tim W. Fawcett, ${ }^{a, b}$ Pieter van den Berg, ${ }^{a}$ Franz J. Weissing, ${ }^{a}$ Justin H. Park, ${ }^{\mathrm{c}}$ and Abraham P. Buunk ${ }^{\mathrm{d}, \mathrm{e}}$

${ }^{a}$ Theoretical Biology Group, University of Groningen, 9750 AA Haren, The Netherlands; ${ }^{\mathrm{b}}$ GRIP, Université de Montréal, Montréal, QC, H3T 1J7, Canada; ${ }^{\mathrm{C}}$ Department of Experimental Psychology, University of Bristol, Bristol BS8 1TU, United Kingdom; ${ }^{\mathrm{d} D e p a r t m e n t}$ of Social Psychology, University of Groningen, 9712 TS Groningen, The Netherlands; ${ }^{\mathrm{e}}$ Royal Netherlands Academy of Arts and Sciences, 1000 GC Amsterdam, The Netherlands. tim.fawcett@cantab.net www.timwfawcett.com pieter.van.den.berg@rug.nl www.rug.nl/biol/theobio f.j.weissing@rug.nl www.rug.nl/biol/theobio j.h.park@bris.ac.uk http://jhpark.psy.bris.ac.uk a.p.buunk@rug.nl www.apbuunk.com

Abstract: Selection on grandparental investment is more complex than Coall \& Hertwig $(\mathrm{C} \& \mathrm{H})$ propose. Patterns of investment are subject to an intergenerational conflict over how resources should be distributed to maximize fitness. Grandparents may be selected to distribute resources unevenly, while their descendants will be selected to manipulate investment in their own favor. Here we outline the evolutionary basis of this conflict.

We applaud Coall \& Hertwig $(\mathrm{C} \& \mathrm{H})$ for highlighting the central role of evolutionary principles in understanding the important and understudied phenomenon of grandparental investment. But we can take their approach further. A proper fitness-based analysis of kin-directed behavior reveals considerable conflict and a richer, more complex picture of the selective forces shaping patterns of investment.

Although equally related to all of their grandchildren, grandparents are not necessarily selected to distribute their resources evenly. The optimal allocation pattern depends on the shape of the curve describing fitness returns on their investment (Fig. 1). If fitness returns are linear (curve A), all distribution patterns yield the same total return; it does not matter whether they spread their investment evenly or favoritize particular grandchildren. If returns are decelerating (B), it always pays to invest in the grandchild with fewest resources, which will tend to promote an even allocation pattern (though see below). If returns are sigmoidal $(\mathrm{C})$, intermediate levels of investment are favored; so grandparents with limited resources might be better off directing them all to one grandchild rather than trying to spread them evenly. Other curves are possible and may favor alternative patterns of investment.

This situation is complicated further by the fact that grandchildren may receive different levels of investment from their own parents (i.e., the grandparents' offspring and sons-/daughtersin-law), putting them at different positions on the returns-oninvestment curve. As an example, consider two grandchildren, $x$ and $y$ (shown in Figure 1), who are first cousins (they share grandparents, but not parents). Prior to any grandparental

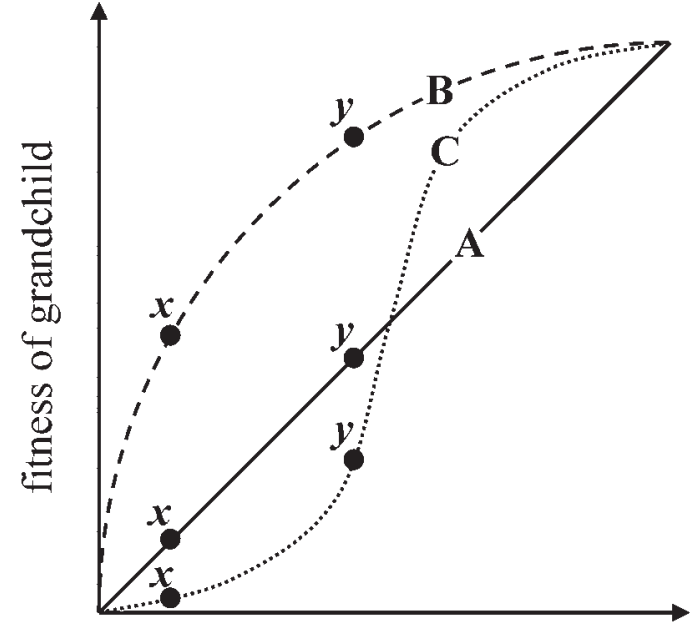

resources invested in grandchild

Figure 1 (Fawcett et al). Three hypothetical relationships between the total amount of resources invested in a grandchild and the expected fitness (lifetime reproductive success) of that grandchild. A (solid line): linear returns on investment; B (dashed line): decelerating returns on investment; C (dotted line): sigmoidal returns on investment. The points $x$ and $y$ refer to two different grandchildren who have previously received different levels of investment from their parents (see text for details).

investment, these individuals have received different amounts of investment from their own parents. Grandchild $y$ is in a relatively privileged position, in that its parents have been able to invest more resources in it than the parents of grandchild $x$. How should the grandparents optimally invest in these grandchildren? The answer depends on the shape of the returns-on-investment curve. If returns are decelerating, grandparents should invest preferentially in grandchild $x$; if returns are sigmoidal, they should favor grandchild $y$; if returns are linear, it does not matter which grandchild they invest in.

Whatever pattern of investment is favored from the point of view of the grandparents, the beneficiaries are unlikely to agree with this. The evolutionary basis for intergenerational conflict over parental investment is well established (Trivers 1974), and the same principles will lead to a similar intergenerational conflict over grandparental investment. Put simply, grandchildren are more related to themselves (coefficient of relatedness, $r=1$ ) than to their siblings $(r=0.5)$ and cousins $(r=0.125)$, and so will seek a disproportionate share of grandparental resources. Their parents will partly support this bias, since they are more related to their own children $(r=0.5)$ than to their nieces and nephews $(r=0.25)$. Thus, as recognized by $\mathrm{C} \& \mathrm{H}$, grandparental investment is not a one-way street; but this is the case even when the investment is not reciprocated. More directly, descendants may be looking to exploit their grandparents' willingness to invest.

How will this conflict manifest itself? The relatedness asymmetry will favor behaviors in the grandchildren and their parents that increase the chances of resources being allocated disproportionately to their own direct line of inheritance. This may entail overt manipulation - for example, direct requests for resources but also more subtle tactics such as maintaining regular contact with the grandparents. Thus, while grandparents could conceivably invest resources in their grandchildren as a way of encouraging support from those grandchildren or their parents (Friedman et al. 2008; Laferrère \& Wolff 2006), a contrasting view is that grandchildren and their parents actively seek contact with the grandparents and provide support as a means of encouraging greater resource investment. 
Of course, grandparents are not passive partners in this process, and will be selected to resist any attempts to divert resources that conflict with their own fitness interests. Typically, grandparents will favor a more equal allocation of resources than their grandchildren, but depending on the returns-on-investment curves and the amount of resources contributed by the parents (Fig. 1), this may not always be the case. Where it pays grandparents to favoritize particular grandchildren, the conflict with those grandchildren will be weaker, while that with the others will be intensified.

Trivers (1974) recognized that intergenerational conflict over care might also impinge on mate choice, and our own work (Buunk et al. 2008; van den Berg et al. 2009) supports this. As explained above, grandparental investment decisions should be sensitive to inequality in the resources their grandchildren receive from other sources. A major determinant of this resource distribution is the care provided by the sons- and daughters-inlaw of the grandparents, that is, the parents who marry into the descendant line. If these in-laws differ markedly in their contributions to parental care, grandparents may adjust their own levels of investment accordingly. But they might also be able to manipulate the resource distribution in their favor at an earlier stage, by actively influencing their offspring's choice of spouse in the first place. Arranged marriages and other forms of control over offspring's mate choice are common across cultures and throughout history (Apostolou 2007) and reveal considerable disagreement over the characteristics of a suitable partner (Buunk et al. 2008; in press). Our evolutionary analysis (van den Berg et al. 2009) confirms that such disagreement can arise from the intergenerational conflict over grandparental investment outlined earlier. Typically, individuals will value caring qualities more highly in their offspring's mates (i.e., their sons-/daughters-in-law) than in their own mate.

In a general sense, we fully support C\&H's first steps towards a theory of grandparental investment, in which evolutionary principles play a prominent role. But for a deeper understanding of investment patterns, we should explicitly consider the details of the selective forces involved. Grandparental investment is not solely in the hands of the grandparents; it is subject to an intergenerational evolutionary conflict in which the fitness interests of three generations are at stake, with each party selected to manipulate the pattern of investment for its own benefit.

\section{Motivating grandparental investment}

\section{doi:10.1017/S0140525X09991622}

\section{Debra Friedman ${ }^{\mathrm{a}}$ and Michael Hechter ${ }^{\mathrm{b}}$ \\ ${ }^{a}$ College of Public Programs, Arizona State University, Phoenix, AZ 85004; ${ }^{\mathrm{b}}$ School of Politics and Global Studies, Arizona State University, Tempe, Arizona 85287-5102. \\ Debra.friedman@asu.edu \\ Michael.hechter@asu.edu}

Abstract: What makes the question of grandparental investment so very interesting is trying to tease out the underlying motivations. Grandparental investment is variable and grandparental altruism, if it exists at all, is also variable. Neither evolutionary theory nor rational choice theory has an easy time explaining this variation, and insight is further impeded by the absence of any compelling empirical studies designed for the purpose of testing alternative explanations of variations in grandparental investment.

At the end of their article, Coall \& Hertwig $(\mathrm{C} \& \mathrm{H})$ call for a comprehensive theory that takes into account evolutionary, economic, and sociological variables. Yet, even if there were such a theory, we might be no closer to a deeper appreciation of grandparents' behavior with respect to their children and grandchildren. Certainly, since the publication of our article (Friedman et al. 2008), in which we also reviewed the available empirical evidence, there have been no theoretically motivated empirical studies. To gather such data would be exceptionally demanding: A comparative test of the relative merits of evolutionary and rational choice (or exchange-theoretic) explanations would demand the study of investment behavior across a number of whole family sets wherein there were at least two children each of whom had at least one child. Furthermore, to be a proper test, the study would have to follow these family sets over time. Given the requirements for this research, we may be engaging in theoretical speculation for quite some time.

$\mathrm{C} \& \mathrm{H}$ advance the theoretical debate by their generous multidisciplinarity, but they muddy it by conflating dependent variables. Differential investment of time, money, and other resources in vivo is distinct from decisions about bequests, and so too is it distinct from opportunity for investment, which is yet different from its potential impact. Implications of grandparental solicitude for the public good in the form of potential policies are definitely premature. (If societies are planning to subsidize grandparental investment, is the outcome better if parents are subsidized for the care of their children rather than subsidizing grandparents for the care of their grandchildren? Or is it more efficient and effective to promote childcare as paid work?) There is a bit of a too-rosy picture of grandparents, as well: Grandparents get older, sick, die, exact an emotional price for their investments, and so on, even if they begin their solicitude pure in their motivations.

As we are collectively waiting for the researchers who will make the investments necessary to gather the data required for robust empirical tests of the major competing theories, there are some purely theoretical debates still to be had. For instance:

(1) Suppose that the evolutionary model were, in fact, a faithful representation of grandparental motivation to invest? According to the model, we can count on parents to maximize the well-being of their children in order to ensure genetic fitness. Why, then, don't grandparents simply invest in their children and assume that their children will do the same, obviating the need for them to invest in their grandchildren, at least under low-risk conditions? Whereas evolutionary theorists may define parental investment as all the resources that parents invest in children to maximize their chance of survival to sexual maturity, it is well known that parents continue to invest massively in post-pubertal children, at least in developed societies (such as paying for their university tuition).

(2) A fair amount is attributed to paternal uncertainty in evolutionary theory (contrary to our own predictions). If grandparents have the choice between investing in a son's children and in a daughter's children, what is the prediction? According to evolutionary theory, why would grandparents with a choice invest in a son's children at all? C\&H note that "the general finding that maternal grandfathers provide more investment to grandchildren than paternal grandmothers ... only held when the paternal grandmothers had other children in whom to invest" (section 3.6, para. 2). Indeed so: grandparental investment is variable across children.

(3) How does empathy add to understanding of differential investment? If there is an inbred empathic capacity, how are we to explain variations in investment? Ditto for altruism.

(4) Finally, the target article's Table 2 can only be taken seriously as evidence of any kind of grandparental impact on grandchildren if it is compared with the impact that any adult investment - related and unrelated adults - have on outcomes. Is a grandparent really better than a fabulous - and well-paid nanny? The assumption that genetic fitness trumps pecuniary incentives is just that - an assumption, and a highly questionable one at that.

Recalling that our theory predicts that grandparents will invest in grandchildren of the child most likely to provide care for the grandparent toward the end of life, here are some hypotheses 
that we would want to add to our model, based on C\&H's discussions:

a. Grandparental investment will be indifferent to the age of the grandchild (see target article, sect. 3.4, para. 1).

b. Grandparental investment is independent of grandparentgrandchild exchange and sensitive to grandparent-child exchange (sect. 4.1).

c. Bequests are motivated by a different calculus than in vivo investments. Grandparents have no motivation to diverge from the equal division norm in death, based on our model.

d. Grandparents who make a clear investment in one set of grandchildren are likely to have fewer depressive symptoms and reduced cognitive impairment (sect. 8.2, paras. $1-3$ ).

All told, this is a topic that is ripe for a productive assessment of the relative merits of evolutionary and rational choice theories of individual behavior. The same is true for the much better appreciated phenomenon of declining fertility in developed societies. $\mathrm{C} \& \mathrm{H}$ advert to the demographic transition in their essay, but fail to point out that there is no satisfactory evolutionary explanation of it. Rational choice theory fares better in this respect (Friedman et al. 1994).

\section{ACKNOWLEDGMENT}

The authors are grateful for the comments of Satoshi Kanazawa.

\section{An evolutionary perspective can help unify disparate accounts of grandparental investment}

doi:10.1017/S0140525X09991634

\section{Michael Gurven and Eric Schniter \\ Integrative Anthropological Sciences Program, Department of Anthropology, University of California-Santa Barbara, Santa Barbara, CA 93106-3210. gurven@anth.ucsb.edu eschniter@gmail.com \\ http://www.anth.ucsb.edu/faculty/gurven/ \\ http://sites.google.com/site/ericschniter/}

Abstract: Coall \& Hertwig $(\mathrm{C} \& \mathrm{H})$ bring attention to alternative accounts of grandparental investment from economics, evolutionary anthropology, psychology, and sociology, which have yet to be reconciled. We attempt to help integrate some of the disparate perspectives by expanding the scope of the evolutionary perspective, highlighting some gaps, and discussing problems with the authors' treatment of grandparents in traditional societies.

Coall \& Hertwig $(\mathrm{C} \& \mathrm{H})$ offer a comprehensive survey of literature pertaining to grandparental "altruism" and call for an integration of disparate perspectives. Although the scope and coherence of a unified theoretical approach are not clearly defined, the authors are to be commended for raising important issues. We argue that evolutionary and rational actor perspectives could be expanded to provide a framework that encompasses both ultimate and proximate-level explanations.

Highly encephalized brains, slow growth, and long lives are derived features of human life history, with juvenile dependency, complex skill development, and grandparenting as key components. If the function of post-reproductive lifespan is to improve fitness of descendant kin, a wide range of cognitive and behavioral traits that focus attention on perceiving and responding to needs of particular kin is expected. Emotions, like motivations, could further modulate behaviors that either benefit or burden particular kin. Psychological studies of wisdom among older adults in modern societies (Baltes et al. 1992) and of kin-favoring dispositions despite age-related physical decline (Carstensen \& Lockenhoff 2003) are consistent with an evolutionary perspective. Norms and institutions might help facilitate delivery of benefits, even when co-residence is unlikely, as codified in inheritance rules. Norms and institutions are considered features of the sociological domain and emotions as part of psychology, yet evolutionary theory and economics are required to make sense of why norms, institutions, and emotions occur in particular forms and expressions. The evolutionary study of emotions and norms is a rich industry.

Evolution has led to a long human lifespan with a substantial post-reproductive phase, yet, despite the adaptive value that grandparenthood must have provided our ancestors, the authors point out a conundrum: Grandparents in the past overlapped with grandchildren for a brief period but with large fitness impact, whereas longer-living grandparents today have more overlap and thereby greater potential to help, but few grandchildren. As a consequence, grandparents in the past increased fitness by reducing infant mortality, but today mostly have only "soft" impacts on well-being and cognition. We feel that (1) the contrast made between past and present opportunity is overstated, and (2) differences in investment patterns depend on marginal benefits of grandparental help, which varies among societies based on differences in fertility, production patterns, co-residence, and inheritance.

Contrary to the statement that grandparental opportunity is strongest today, evidence suggests that the opportunity to help grandchildren was higher among our hunter-gatherer ancestors. First, while mortality and fertility are lower today, age at first marriage is also much later, and so Westerners become grandparents about 12 years later on average than do hunter-gatherers. Thus, the average number of years lived as a grandparent may not be very different between groups (Table 1). Second, hunter-gatherers are more likely to be co-resident with grandchildren and the total number of grandchildren to potentially impact is higher (fertility of hunter-gatherers is $4-8$ births).

Third, support for the idea that grandparents in traditional societies increase fitness has relied on historical demographic datasets to measure the impact of their presence on early life mortality. Anthropological studies of grandparental contributions focus primarily on food production of older adults. To our knowledge, caloric production (or any other grandparental behavior) has yet to be causally linked to child welfare in any of these studies. Despite the popularity and importance of the Grandmother Hypothesis and alternate explanations of post-menopausal lifespan, all studies of grandparental impacts on kin fitness are indirect, based on whether a grandparent was alive or dead, or in rare cases, co-resident, in a given year. To what extent is the early weaning of infants, higher infant and child survivorship, and earlier reproduction, influenced by grandparents? Until these pathways are studied, phenotypic correlations may confound any observed positive relationship between living grandparents and kin survivorship or fertility. Without an understanding of the proximate mechanisms by which grandparents likely improved kin welfare, detailed predictions about what grandparents should be doing today (and whether their behavior is maladaptive) are difficult to make.

Fitness is impacted by accumulating and transferring material, embodied, and relational wealth, and societies vary in the extent to which each of these is inherited and needed for cultural and biological "success" (Borgerhoff Mulder et al. 2009). Grandparents should facultatively adjust their aid behavior where they can have the highest marginal benefit at lowest personal cost. Whether in small-scale societies or modern post-industrial ones, we suspect that the greatest impact of grandparents may be realized during rare, but fitness-relevant, periods. The authors describe postpartum depression and teenage pregnancy in modern societies as examples. We mention a few others here based on ten years of fieldwork among Tsimane forager-horticulturalists of Bolivia. Tsimane grandparents are often primary caretakers when parents die: $17 \%$ of adult Tsimane interviewees had a parent die before age 18 , and $19 \%$ of these went to live with a 
Commentary/Coall \& Hertwig: Grandparental investment

Table 1 (Gurven \& Schniter). Demographic parameters for hunter-gatherers and modern populations

\begin{tabular}{|c|c|c|c|c|c|}
\hline Population & $\begin{array}{l}\text { Age at first birth } \\
(\mathrm{AFB})\end{array}$ & $\begin{array}{l}\text { Remaining life } \\
\text { expectancy, }\left(\mathrm{e}_{\mathrm{AFB}}\right)\end{array}$ & $\begin{array}{l}\text { Age at first grand- } \\
\text { parenting (AFGB) }\end{array}$ & $\begin{array}{l}\text { Remaining life } \\
\text { expectancy, }\left(\mathrm{e}_{\mathrm{AFGB}}\right)\end{array}$ & $\begin{array}{l}\text { Total fertility rate } \\
\text { (TFR) }\end{array}$ \\
\hline Hunter-gatherers & 19 & 36 & 38 & 25 & 5.4 \\
\hline $\begin{array}{l}\text { Acculturated hunter- } \\
\text { gatherers }\end{array}$ & 19 & 44 & 38 & 30 & 5.5 \\
\hline Spain, 2002-2007 & 29.3 & 53 & 58.6 & 26 & 1.28 \\
\hline United States, 2004 & 25.0 & 54 & 50 & 31 & 2.09 \\
\hline
\end{tabular}

Data Sources: Hunter-gatherers: Gurven and Kaplan (2007), Hewlett (1991); Spain: Instituto Valenciano de Investigaciones Económicas (Goehrlich, http://www.ivie.es); United States: National Center for Health Statistics (http://www.cdc.gov/nchs/data/nvsr/nvsr57/ nvsr57_14.pdf).

grandparent (9.8\% with maternal, $9.0 \%$ with paternal). Even when not holding leadership positions, older adults mediate conflicts between different kin factions, which helps to promote coordination in activities so as to more efficiently reap gains from divisions of labor and economies of scale. While the "soft" impact of grandparents in traditional societies has not been described, we suspect that further inquiry may reveal that the marginal impact of grandparents is not primarily in the form of calories. Grandparents are named as important transmitters of Tsimane skills and knowledge (Gurven \& Kaplan 2008; Schniter 2009); they account for $8 \%$ of identified contributors to early-life skill acquisition. They are twice as likely to be named for rare but important skills, such as making pottery, punishing bad behavior, singing traditional songs, and telling old stories and myths.

Finally, an evolutionary perspective emphasizes not only grandparents' cumulative fitness impacts, but also the increasing costs on descendants with age. Few hunter-gatherers and horticulturalists live beyond the seventh decade of life. Among Tsimane, we observed that grandparents in their 70's no longer make net-transfers of food to grandchildren. Whether the complementary contributions listed above are sufficient to slow the decline in utility is an open question, but we suspect that net utility is negative by the late 70 's. In pre-industrial societies where production is costly and resource competition is high, geronticide and neglect are commonly practiced (Maxwell et al. 1984). Elderly populations today, whose knowledge and traditions may be devalued, given rapid cultural change, show increasing evidence of neglect and abuse (Lachs \& Pillemer 2004). Intervention programs that focus on the marginal benefits grandparents can offer may be helpful for strengthening familial care networks and building communities (Denham \& Smith 1989). The total value of grandparents as fallback caretakers, educators, mediators, storytellers, and as sources of wisdom is too important as social insurance to risk losing, even in modern societies.

\section{Grandparental transfers and kin selection}

doi:10.1017/S0140525X09991646

\section{Raymond Hames \\ Department of Anthropology, University of Nebraska, Lincoln, NE 68588. rhames@unl.edu \\ http://www.unl.edu/rhames}

Abstract: In the analysis of intergenerational transfer, several improvements can be made. First, following kin selection theory, grandparents have kin other than grandchildren in which to invest and therefore any investigation into grandparents should take this perspective. Secondly, how transfers actually enhance the survivorship of younger relatives such as grandchildren must be better measured, especially in the ethnographic literature. Finally, the problem of indirect investments or targeting must be considered.

Coall \& Hertwig $(\mathrm{C} \& \mathrm{H})$ present a wonderfully comprehensive and admirable review on the investing roles that grandparents play in traditional and modern societies. I would direct any of my students to this article if they were beginning research on the topic. Given that the article is so comprehensive, I restrict my comments to the history of this research in anthropology, especially as it relates to kin selection theory and some measurement issues that would better assess the ways in which grandparents matter.

There are several strands of research in evolutionary anthropology that deal with the role that grandmothers may play in enhancing the fertility of their children and survival of their grandchildren, beginning with the work of Turke (1989) and Kaplan (1994) on intergenerational resource transfers, the literature on "helpers at the nest" (for a review, see Hames \& Draper 2004), as well as reviews of the grandmother literature (Sear \& Mace 2008). To a limited extent, the theme of extended family intergenerational transfers is picked up by the authors in section 8.2 (under the heading of the "one-way street?") and elsewhere. Turke and Kaplan criticized the work of the influential development demographer Caldwell (1976), who argued that high fertility in the developing world is a kind of social security mechanism whereby the elderly through high fertility produce children and eventually grandchildren who will support them in their old age. This strategy functions as insurance in social systems that lack effective social security. Turke and Kaplan note that Caldwell's model is a challenge to evolutionary demography and kin selection theory because the fitness concerns of family members, especially grandparents, should lead to a flow of wealth and resources from those who have low reproductive value to those who have greater reproductive value, adjusted by the coefficient of relatedness. If the flow were opposite, from younger to older, the fitness of the younger would be reduced, as well as the inclusive fitness of the older generation. The literature $\mathrm{C} \& \mathrm{H}$ review tends to support Turke and Kaplan's view in the modern context, but we need more research in the developing world, as exemplified by the research reviewed by Sear and Mace (2008).

The next research thread moved the focus from the extended family to a close examination of the impact of grandparents and was initiated by Hawkes and colleagues, beginning with their work on Hadza grandmothers (Hawkes et al. 1989). These researchers argued that menopause was designed by natural selection to channel resources to grandoffspring. This insight generated a large amount of high quality research on grandmother effects on the survivorship of grandoffspring and the fertility of their children. $\mathrm{C} \& \mathrm{H}$ point out that much of this research is summarized in Hrdy's conceptualization of communal breeding (Hrdy 2005a) and in the general literature on helpers at the nest. 
Viewing grandparental investment from a kin selection perspective would be methodologically useful for several reasons. First, grandparents have a wide variety of lineal and collateral kin as potential recipients of their benefits. It seems that research might be more profitably centered on how grandparents allocate their resources to all kin, including grandchildren, instead of focusing on how much grandchildren have received from grandparents. The grandparent to grandchild focus may hide transfers that grandparents may make to other kin. Although grandchildren are related by one-quarter to grandparents, the latter are related to their nephews and nieces to the same degree, even though the reproductive value of grandchildren is likely to be higher. Secondly, another problem that needs to be methodologically addressed is what can be called indirect transfers or the problem of targeting (Hames 1987). Parents may be motivated to invest in their children because they know that such investments will be passed on to their grandchildren. For example, a monetary transfer to a child with a new-born grandchild may be motivated by a desire to invest in the grandchild by allowing its parents to make purchases that ultimately enhance the wellbeing of the grandchild. I would also add that the character of transfers should be sensitive to the age of the grandchild, such that initial investments may be in the form of direct grandparental care such as babysitting, to monetary transfers later in life for assistance in higher education.

Most of the studies in anthropology are demographic, in that they show correlations between grandmother presence and either enhanced survivorship of grandchildren or greater fertility of mothers. It is clear that we need more observational or more detailed survey research to learn exactly what grandparents do to cause these effects. This is particularly true of much of the ethnographic research in traditional societies, but much less true in wealthy countries, as $\mathrm{C} \& \mathrm{H}$ show. In ethnographic research, how grandchild survivorship and offspring fertility is achieved by the presence of grandparents is largely speculative, and $\mathrm{C} \& \mathrm{H}$ rightly note that such research only "highlight[s] potential behaviors that promote these beneficial effects" (sect. 2.5). Are mothers, for example, spared child-care activities to enhance their productivity, or do Hawkes et al.'s "hardworking Hadza grandmothers" (Hawkes et al. 1989) produce food that is transferred to their daughters' households to enhance grandchild growth and development? These crucial sorts of investment could be measured in a variety of ways, such as carefully tracking data on actual transfers or through time allocation data (time diaries or direct observation). These measures are particularly important because they more accurately gauge the exact kinds of benefits from grandparents to offspring and their likely effects.

\section{Grandparental investment facilitates harmonization of work and family in employed parents: A lifespan psychological perspective}

\section{doi:10.1017/S0140525X09991658}

\section{Christiane A. Hoppmann ${ }^{a}$ and Petra L. Klumb ${ }^{b}$ \\ ${ }^{a}$ Department of Psychology, University of British Columbia, Vancouver, BC, Canada, V6T 1Z4; ${ }^{\mathrm{b}}$ Department of Psychology, University of Fribourg, 1700 Fribourg, Switzerland. choppmann@psych.ubc.ca petra.klumb@unifr.ch}

Abstract: The target article emphasizes the need to identify psychological mechanisms underlying grandparental investment, particularly in low-risk family contexts. We extend this approach by addressing the changing demands of balancing work and family in lowrisk families. Taking a lifespan psychological perspective, we identify additional motivators and potential benefits of grandparental investment for grandparents themselves and for subsequent generations.
Coall \& Hertwig $(\mathrm{C} \& \mathrm{H})$ discuss the changing role of grandparents in industrialized societies and the scarcity of research on grandparental investment in low-risk families. They develop a comprehensive model that generates novel hypotheses regarding the psychological mechanisms underlying grandparental investments. Particularly, they emphasize the benefits of moving beyond classic fitness indices and including psychological outcomes. Taking a lifespan developmental perspective, we offer additional reasons for focusing on grandparental investment as a key resource that may promote the harmonization of work and family responsibilities in low-risk families, thereby enhancing the developmental outcomes of children and grandchildren.

Past research shows beneficial effects of grandparents on the health and survival of grandchildren in historical or contemporary agricultural societies. It is an open question whether and how these benefits extend to today's industrialized societies. Evidence from high-risk families suggests that grandparents constitute a protective resource for their grandchildren. Little is known, however, about low-risk families. This research gap needs to be filled for at least two reasons. First, many low-risk families struggle to meet increasing demands at work and at home. For instance, employers expect increasing flexibility and mobility of employees (Klumb \& Gemmiti, in press), while institutional childcare is rigid and not always available. The demands of juggling work and family life can negatively impact the health and well-being of parents and children alike (Strazdins et al. 2006). Research on low-risk families will thus enable us to identify factors that enhance health and well-being in large segments of the population. Secondly, in some European countries, the increased demands of juggling work and family life have coincided with dramatic decreases in fertility. In Germany, for example, childlessness increases with education and is highest among university graduates (Duschek \& Wirth 2005). In other countries, too, women's careers come at a cost to their private lives (Galinsky et al. 2003). Reversing such demographic trends is difficult, but seems possible (Myrskylä et al. 2009). Grandparental support may be one important piece in this picture. By supporting working parents, grandparents can serve as buffers against the negative consequences of parental stress on children, and, in regions with scarce childcare facilities, they may be an important factor in women's decisions to have children (Hank \& Kreyenfeld 2003). Hence, there may be an important indirect pathway through which grandparental investment benefits grandchildren, namely, by alleviating stress in the middle generation. Further investigation of this pathway seems promising, because, as $\mathrm{C} \& \mathrm{H}$ point out, the overlap between the lives of grandparents and grandchildren is currently at its peak.

$\mathrm{C} \& \mathrm{H}$ develop intriguing hypotheses regarding the role of psychological mechanisms (e.g., empathy) in motivating grandparental investments. We would like to offer an additional perspective on the antecedents and consequences of grandparental investments by introducing the concept of developmental tasks to the discussion (Erikson 1966). The concept of developmental tasks represents a system of age-related, socially valued themes (Havighurst 1953). Generativity is a key task in the second half of the adult lifespan and is characterized by a sense of responsibility for subsequent generations, in particular a concern to guide the next generation (Erikson 1966). Generativity is strongest with respect to one's own offspring and may thus be an important motivator for grandparental investments. For example, by participating in the care of grandchildren, grandparents may be able to help their adult children to reconcile their work and family responsibilities while contributing to the raising and guiding of their grandchildren. The literature also proposes that the realization of developmental tasks fosters personal growth and wellbeing. This suggestion is supported by observations that adult children's attainments and achievements are central for the well-being of older parents (Ryff et al. 1994). Hence, generativity may serve not only as an important motivator of grandparental investment benefiting younger generations, but also as a source 
of well-being for grandparents themselves. We would like to illustrate possible mechanisms by drawing on data from a recent study on the pursuit of work and family goals in employed parents with preschool children (Hoppmann \& Klumb 2004; 2006). The study asked 84 highly educated employed parents in Berlin to report their work and family goals, rate time- and strategy-based conflicts between their goals, and indicate access to grandparental childcare. In addition, we recruited 31 of the grandparents who provided childcare and asked them to complete the Loyola Generativity Scale and to report their own goals, their respect for their adult children's goals, and affect quality. As the Berlin metropolitan area has a good childcare infrastructure, none of the grandparents were daily caregivers. Findings indicate that grandparental generativity, goals related to wanting to leave an imprint on others' lives and wanting to feel needed, and respect for adult children's goals were positively associated with grandparents' desire to provide childcare. These associations held when we controlled for living distance, grandparental employment status, health, and age. Hence, generativity seems to be an important motivator of grandparental investments. In addition, we found that grandparental childcare provision and adult children's pursuit of work and family goals were positively related to grandparental positive affect. Hence, grandparental investments and adult children's achievements seemed to be important sources of well-being in this sample. Finally, we examined the impact of grandparental childcare on their adult children. We found that grandparental childcare buffered the negative effects of adult children's goal conflicts upon their pursuit of work and family goals. This finding suggests that grandparental investments may indeed be a key resource facilitating the harmonization of work and family lives in adult children.

Future research on the buffering effects of grandparental investments should differentiate the needs created by different types of stressors. Grandparental support may be most effective when it matches those needs (Cohen \& Wills 1985). For example, grandparental childcare may help employed parents struggling against time scarcity, but it may not work as well if employed parents are emotionally challenged (Frone 2000). Furthermore, there may be circumstances in which grandparental support is a mixed blessing. Although grandparents may be a powerful resource capable of offsetting stress in younger generations, lifespan research shows that unasked-for support can be perceived as a suggestion of incompetence and thus a threat to the self (Smith \& Goodnow 1999). Hence, grandparental support may convey mixed messages that can strain relationships with children. We would therefore like to emphasize the importance of determining the specific conditions under which grandparental investments are beneficial for all concerned.

\section{Continuity between pre- and post- demographic transition populations with respect to grandparental investment}

\section{doi:10.1017/S0140525X0999166X}

\section{Brad R. Huber \\ Department of Sociology and Anthropology, College of Charleston, Charleston, SC 29424.}

HuberB@ cofc.edu

http://huberb.people.cofc.edu/

Abstract: This commentary suggests that there is more continuity in pre- and post-demographic transition populations with respect to grandparental investments than is assumed by Coall \& Hertwig $(\mathrm{C} \& \mathrm{H})$. Recent research employing high-quality data supports the claim that sex-biased grandparental investments are likely to exist in industrialized societies, and that the economic status of grandparents is related to their long-term fitness.
I congratulate Coall \& Hertwig $(\mathrm{C} \& \mathrm{H})$ for their thorough and rigorous analysis of grandparental investment. I agree there is a need for a more comprehensive theoretical framework of grandparental investment, one that explains the proximate and ultimate causes of investments, and that takes into account institutional arrangements, such as the presence of a state-financed welfare system. My comments focus on three claims the authors make.

The authors claim that the impact of grandparental investments in contemporary industrialized societies is relatively small when measured by classic indicators of fitness, such as number of grandchildren and grandchild mortality. This point needs some qualification. In post-demographic transition populations where people are living under low-risk conditions, direct grandparental investments will always have relatively little impact on the number and mortality of grandchildren when compared to the impact of direct grandparental investments in societies with high fertility and mortality rates. This is because the probability of grandchildren being born or dying is so much lower in post-demographic transition populations than it is in pre-demographic transition populations. Even if grandparents made very large direct investments in their grandchildren, this will have relatively little impact on the number and mortality of grandchildren in industrial societies.

However, the indirect investments grandparents make in industrialized societies do make a tremendous difference in the likelihood of their children and grandchildren surviving and reproducing. Here, I am referring primarily to the institutions that grandparents, and other taxpayers, support (e.g., Department of Health and Human Services) that create and maintain the relatively low fertility, low mortality conditions under which their children and grandchildren live. Although it is difficult to measure the impact indirect grandparental investments have on classic measures of fitness in contemporary industrialized societies, I would argue that their impact is very substantial.

C\&H's position regarding the application of the TriversWillard hypothesis to parental and grandparental investments in industrialized societies also merits comment. I agree with them that the evidence of differential resource allocation to sons and daughters by parents in industrialized countries is conflicting, and that there is currently no convincing evidence of sexbiased grandparental investment in humans. However, researchers may have been looking for evidence in the wrong places. In the case of parental investment, Rosemary Hopcroft (2005, p. 1116) notes that researchers generally focus on infants and relatively young children. In her opinion, parental biases in investments for this age group are unlikely in a relatively wealthy society such as the United States.

That is, there are abundant resources available for parents to be able to make these early investments in all children regardless of sex. Evolutionary theory suggests that in such a situation of unusual abundance all children, irrespective of sex, will be highly valued and invested in by their parents... [Status] attainment in American society requires much more than just investments in children as babies and adolescents; it requires ongoing investments in very lengthy periods of education and occupational training. . It is provision of support over such long periods of time that is the scarce resource in American society... I suggest, therefore, that the focus of studies testing the resource allocation version of Trivers-Willard should be sex differences in parental provision of access to extensive training and education and encouragement of this process. (Hopcroft 2005, p. 1116)

Using years of education as an indirect measure of parental investment, and cumulative U.S. General Social Survey data, Hopcroft found support for the Trivers-Willard hypothesis in the United States. Sons of high-status fathers attain more education than daughters do, daughters of low-status fathers attain more education than sons, and high-status men have more sons among their biological offspring than do lower-status individuals. 
In light of this finding, I am optimistic that researchers will eventually find evidence for sex-biased grandparental investments in industrialized societies. Promising avenues of research may include the financial and social investments made by grandparents in their grandchild's education, occupational training, business endeavors, and so forth.

My final comment concerns C\&H's view that the poor now out-reproduce the wealthy in post-demographic transition societies. Has the availability of effective contraception really severed the link between status and reproduction in modern human societies? One of the challenges of evaluating this question is having the right kind of data. I again look at Hopcroft's work on reproductive success in the contemporary United States (Hopcroft 2006, p. 107) to illustrate my point. She used a representative sample containing data that made a distinction among an individual's biological, step, and adopted children. Previous research on reproductive success in the United States. did not make these distinctions and tended to rely on data that only report female fertility or the number of children in a household. With data that likely includes all, and only, the living biological children of men and women, Hopcroft (2006) found that highincome men have more biological children than do low-income men and high-income women.

There is also reason to believe that the economic status of grandparents in at least one industrialized society is positively correlated with their long-term fitness, as measured by number of grandchildren. Recently, Anna Goodman and Ilona Koupil (2009) reported their research findings on the social and biological determinants of reproductive success in Sweden during the 20th century. They used high quality, multi-generational data on a cohort born from 1915 to 1929, a time when the Swedish demographic transition was largely concluded. Goodman and Koupil had many interesting findings. The main one regarding grandparental socioeconomic status and reproduction follows:

The effect of SEP [family socioeconomic position] is interesting in highlighting the potential. .. continuity [between pre- and post-demographic transition populations with respect to]... the determinants of reproductive success. The continuity is illustrated by the positive correlation in both sexes [of grandparents] between higher SEP and a greater number of descendents. This is consistently observed in traditional populations (Hill \& Kaplan 1999; Low 2000) and, although evidence in modern populations is more variable (Clarke \& Low 2001), our study adds to the evidence that the demographic transition does not necessarily erase the relationship between access to resources and reproductive advantage. (Goodman \& Koupil 2009, p. 339)

\section{Fitness effects of grandparental investments in contemporary low-risk societies}

\section{doi:10.1017/S0140525X09991671}

\section{Ralf Kaptijn and Fleur Thomese}

Faculty of Social Sciences, Vrije Universiteit Amsterdam, 1081 HV Amsterdam, The Netherlands.

rwj.kaptijn@fsw.vu.nl

gcf.thomese@fsw.vu.nl

Abstract: Coall \& Hertwig $(\mathrm{C} \& \mathrm{H})$ suggest that, because grandparental investments do not impact on child mortality in low-risk societies, fitness effects are not plausible any more. We found that grandparental investments could very well alleviate contemporary constraints on fertility. Cultural factors may influence both the occurrence and impact of grandparental investments.

Coall \& Hertwig $(\mathrm{C} \& \mathrm{H})$ argue that in contemporary affluent societies grandparental investments are no longer likely to have a substantial positive effect on classical fitness indicators like child mortality. However, child mortality is only one component of fitness. Other components such as fertility and age at first reproduction have gained more importance in modern societies. In these societies, grandparental investments may relieve some of the important impediments on women's reproduction and in this way have a positive influence on fertility. Recent, yet unpublished, research supports this hypothesis.

Even though under modern conditions almost all children stay alive after birth, there are considerable impediments to women's reproduction. Partly these impediments have biological causes and are not limited to modern societies. Other impediments are more typical for contemporary societies. First, as C\&H indicate (sect. 5.3.), the parental investments required to enable a child to compete with its peers have increased exponentially. These high costs of children limit the number of children the parents can raise. Second, paid employment and motherhood are hard to reconcile. When women are confronted with the dilemma of combining work and motherhood, they tend to reduce their fertility. This phenomenon is reflected in total fertility rates, which generally are lower in countries where work and motherhood are less compatible (Hoem 2008; McDonald 2000). Third, the acceptance and large-scale use of contraceptives have made fertility subject to conscious choice. In liberal societies, women's lifestyle preferences have a bigger impact on fertility decisions. Women's preferences are heterogeneous and some women prefer to be "childfree" (Hakim 2002; 2003).

The diverse impediments that women and couples face are likely to affect their reproductive success. It is our hypothesis that grandparental investments, especially investments in the form of childcare, alleviate these impediments and thus have a positive impetus on fertility. By reducing some of the burden of raising children, grandparental investments may lower the costs of a child for the parents. Grandparental childcare may also ease women's dilemma of combining paid employment and motherhood, and give the mother more possibilities to spend free time without the children. We tested this hypothesis in a representative sample of the contemporary Dutch older population for which information on grandparental babysitting, as well as the fertility of their children, was available. In this prospective study (yet unpublished), we found that grandparental childcare support has a positive effect on their children's fertility. This suggests that grandparental investments may also positively affect their children's fitness in a contemporary affluent society.

A positive relationship between grandparental investments and children's fertility on an individual level is not at odds with a negative relationship between grandparental investments and fertility on a population level as reported by $\mathrm{C} \& \mathrm{H}$ (sect. 8.3.), but rather points to the importance of the institutional context in which grandparental investments take place. It may well be that on a country level, grandparental childcare does not outweigh fertility constraining factors. Our findings are limited to a Dutch population and do not allow conclusions on the role of contextual factors. But, like $\mathrm{C} \& \mathrm{H}$, we think this is an important new field of inquiry. Besides C\&H's emphasis on the importance of economic and institutional factors, we want to stress that cultural factors may also be very important, both in shaping the preferences for care from grandparents, and in its impact on fertility.

The literature on European welfare state regimes (EspingAndersen 1990; Pfau-Effinger 2005) typically links the availability of care to cultural traditions about the role of women and kin in providing care. Roughly speaking, the farther south one gets, the greater the responsibility of women for their children, and the greater the reliance on kin for providing care. The northern countries provide more formal arrangements. The Netherlands stands out for its relatively high availability of child-care in combination with a strong preference of many working women for care from grandparents (Portegijs et al. 2006). This suggests first that the reliance on grandparents may be informed by cultural preferences, which are not only reflected in the institutional care arrangements, but also in the choices individual parents make. 
Second, from an evolutionary perspective, the main question would be how child-care arrangements affect fertility. Does it make a difference if grandparents take care of the children, as compared to non-kin or even relative strangers? Or have our collective institutions simply taken over the role of grandparents in our evolution, as $\mathrm{C} \& \mathrm{H}$ suggest? The latter could be the case if we see our social institutions and society at large as an extension of the kin groups that presumably existed when the grandmother effect first emerged in humans. Recent findings indicate that fertility may be increasing in better-developed welfare states (Myrskylä et al. 2009), and older generations often use the transfers they receive from the welfare state to invest in their children (Kohli 1999). This suggests that the mechanisms behind intergenerational transfers are similar at both macro and micro levels.

However, C\&H show that most evolutionary perspectives on grandparental investments would predict a fitness advantage of taking care of grandchildren. When, as in the Dutch case, grandparents are willing to provide this care, and many parents prefer such care over other possibilities, it seems logical to expect that it also makes a greater difference for the parents' fertility. In that case, cultural preferences could reflect evolutionary advantages of grandparental care. The question then is if and why cultural differences occur in the relevance of grandparents for fertility. We have no answers yet, and at this moment we only have one study to suggest that grandparents do matter for fitness in lowrisk countries. We hope this does make a case for further research on grandparental fitness effects, either by looking into the parents' situation in more detail, or by comparing data from countries with contrasting child-care and cultural backgrounds.

\section{Intergenerational transfers and the cost of allomothering in traditional societies}

\section{doi:10.1017/S0140525X09991683}

\section{Karen L. Kramer \\ Department of Human Evolutionary Biology, Harvard University, Cambridge, MA 02138. \\ kkramer@fas.harvard.edu}

Abstract: The question of why helpers help is debated in the cooperative breeding literature. Recent reevaluations of inclusive fitness theory have important implications for traditional populations in which the provisioning of young occurs in the context of intergenerational transfers. These transfers link older and younger generations in an economic relationship that both minimizes the demand for help and the cost of helping.

Grandparental assistance in helping to raise their children's young is common in many industrialized and traditional societies today, and is hypothesized to be important in the evolution of modern life histories (Hawkes \& Paine 2006; Hawkes et al. 1997). Coall \& Hertwig (C\&H) make a compelling case, both for integrating evolutionary perspectives on grandparenting with economic and sociological views, and for moving evolutionary models forward to explain grandparenting under post-demographic transition conditions. In doing so, they raise a number of salient points about grandparenting specifically and allocare in general.

Humans have been characterized as cooperative breeders, a reproductive strategy in which mothers routinely rely on the help of nonparental individuals to raise young. Because allomothers divert energy and resources to another's reproductive success, it presents an evolutionary puzzle. In addressing this, much of the cooperative breeding literature has centered on the question of why helpers help. C\&H evaluate grandparenting and its altruistic basis in industrialized populations. My comment focuses on this question in traditional human societies.

Hamilton's Rule articulates the conditions under which altruism and cooperation might evolve and provides a heuristic means to view allocare as an adaptive behavior. While Hamilton's Rule has broad appeal as a framework to understand cooperative breeding, the challenge has been to operationalize measures of costs and benefits. Recent research has questioned whether the cost of helping may be overstated (Clutton-Brock 2002), and whether kin-biased behaviors may be motivated by factors other than, or in addition to, kin selection (de Waal 2008; Silk 2004). These reevaluations are particularly prescient for humans living in subsistence economies where the provisioning of young occurs in the context of intergenerational transfers.

Because fitness payoffs are often time-delayed, $\mathrm{C} \& \mathrm{H}$ point out that kin selection per se may be insufficient to motivate helping behaviors (de Waal 2008). In contrast to the psychological mechanisms overviewed in the target article, an alternative mechanism may lie in intergenerational transfers of food and labor that characterizes subsistence economics. The human diet consists of a wide variety of foods, most of which require processing and specialized technology. In all traditional societies, resources and labor are pooled and transferred intergenerationally, but also bidirectionally (Kramer 2005b; Lee \& Kramer 2002). These transfers flow downward from older to younger generations, but also upward from children to adults. Rarely does any individual of any age do all of the tasks necessary to grow, survive, and reproduce. This economic interdependence affects helping behaviors in two ways. Children growing up in transitional economies are not as costly as they are often characterized, and bidirectional transfers link older and younger generations in an economic relationship that minimizes the opportunity cost to help.

Because dependency of human young extends into juvenility, the energetic burden of supporting children often is assumed to increase in step. While children growing up in industrialized societies are "gas guzzlers," they are not so in many traditional societies (Bliege Bird \& Bird 2002; Blurton Jones et al. 1997; Cain 1977; Kramer 2005b; Nag et al. 1978). Juveniles have a complex relationship with their caretakers. They may not have the skills and body strength to perform all of the tasks necessary to survive, but in most traditional societies children provide some portion of what they need. For example, Hadza children provide $50 \%$ of their caloric requirements by the age of five during some seasons (Blurton Jones et al. 1989). When subsistence work is defined to include processing and household tasks, Maya girls produce $50 \%$ of what they consume by the age of six (Robinson Sullivan et al. 2008). But juveniles are also able to overproduce at some tasks - fetching water, harvesting, fishing, collecting shellfish, and foraging for fruit and nuts are good examples. At these tasks, children may meet not only their own consumption, but their surplus production contributes to the "common pot" (sensu Stecklov 1999) and is redistributed among members of the sharing group. Although a greater cross-cultural sample is needed, where bidirectional transfers have been quantified, children's subsistence effort and upward transfers allow mothers to raise more children than they otherwise could provision on their own (Kramer 2005a; Lee \& Kramer 2002). In societies where children make economic contributions, not only do children underwrite part of their cost, but others, including older individuals, benefit from their transfers as well.

Older adults in many traditional forager and agricultural societies produce surpluses that exceed the level of their own consumption. Although they are net producers (Hawkes et al. 1989; Kaplan 1994), they too rely on exchanges from others, including younger generations. In subsistence forager and agricultural economies where the time and effort spent in allo-provisioning is embedded in self-provisioning tasks (e.g., the time a grandmother spends collecting roots or weaving a hammock that she shares with a grandchild), the cost to subsidize grandchildren may be relatively low. Although the intergenerational 
Commentary/Coall \& Hertwig: Grandparental investment

transfer literature has focused on unidirectional flows and net costs, subsistence economies function in a more nuanced and bidirectional manner. Grandparental helping behaviors such as childcare are clear examples of altruistic allocare - activities that benefit another but not the helper - and may be explained by kin selection. However, in humans much of what helpers provide is food and other resources to juveniles. Because provisioning juveniles occurs in the context of general food sharing and labor cooperation and helpers also receive resources from others including those they help, it introduces potential mutualistic benefits and other motivations..

In natural fertility populations, the help mothers receive has been associated with younger ages at first birth, shorter birth intervals, and higher offspring survival (Flinn 1988; Hawkes et al. 1997; Kramer 2004; Turke 1988). Assistance, which traditionally came from grandparents and others, is today further augmented by governments and institutions. In many natural fertility populations today, through external sources of nutritional and medical assistance child survival has increased concomitant with a rise in birth rates (Dyson \& Murphy 1985; Kramer \& Greaves 2007; Kramer \& McMillan 2006; Romaniuk 1980). In post-demographic transition populations childbearing occurs much less often in a kin-based context. As generational time lengthens and families are more prone to geographic dispersion, childrearing support networks often diminish or evaporate. Even though families are small, help from others often is still essential, but support shifts to nonkin-based assistance, paid childcare or institutional assistance. An interesting outcome is that, although mothers make allocation decisions and rely on help, the central evolutionary question about why helpers help is no longer pertinent if caretakers are paid or otherwise compensated. In post-demographic transition populations, while traditional grandparental roles may be supplanted, $\mathrm{C} \& \mathrm{H}$ point out that grandparents continue to have an important effect on the fertility decisions for working mothers and on their grandchildren's well-being.

\section{Population aging and the economic role of the elderly: Bonanza or burden?}

\author{
doi:10.1017/S0140525X09991695
}

\section{Ronald D. Lee \\ Department of Demography, University of California-Berkeley, Berkeley, CA 94720. \\ rlee@demog.berkeley.edu \\ http://www.ceda.berkeley.edu/peoplenew/rlee.html}

Abstract: As societies industrialize, the age profile of consumption tilts strongly toward the elderly, while elder labor supply drops. Low fertility and long life lead to population aging. For millennia, material resources have, on net, flowed downward from older to younger within populations, but now in many rich societies net flows have reversed and go upwards from young to old.

The population share of grandparents and other elderly is rising around the world as a result of low or falling fertility and rising longevity, a share which, even in today's oldest countries, is projected to double or triple in coming decades. From the perspective of hunter-gatherer societies, these abundant elderly should be a bonanza for "gas guzzling children" and their parents. But a funny thing has happened. The economic role of the elderly has been radically transformed in rich industrial nations relative to contemporary hunter-gatherer groups, with low-income agricultural societies intermediate.

Figure 1 plots the difference between the amount consumed at each age and the amount produced (measured as labor income) for average individuals. This difference is the "life cycle deficit," or LCD. The hunter-gatherer estimate is an average for the Ache, Piro, and Macheguenga (Kaplan 1994) and !Kung (Howell, in press). The averages for rich countries (Japan, United States, Sweden, Finland) and for poor countries (Kenya, Philippines, Indonesia, and India) are taken from the international collaborative National Transfer Accounts project

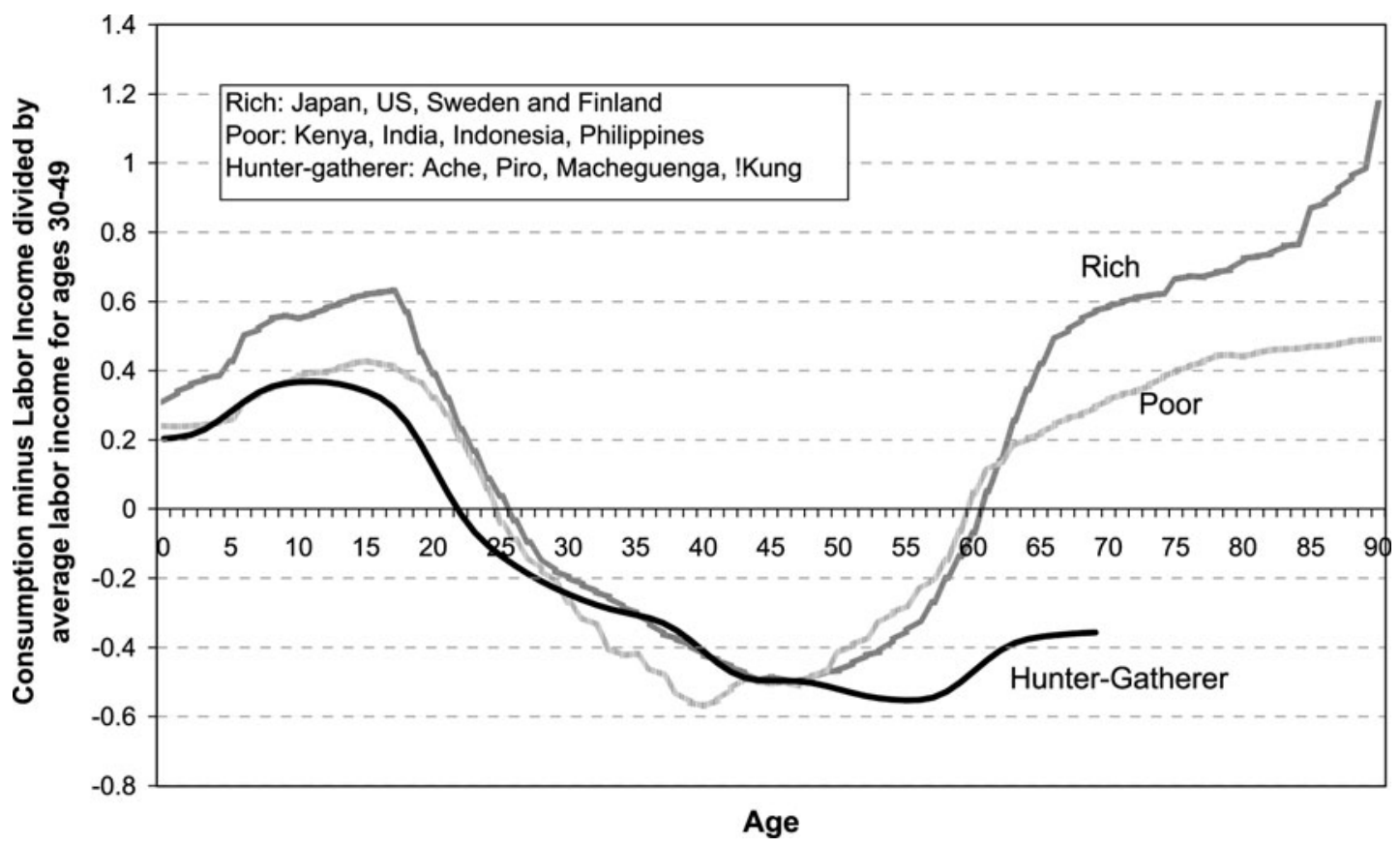

Figure 1 (Lee). Data for rich and poor countries taken from the National Transfer Accounts Project (NTA); see NTAccounts.org.

Data for the Ache, Piro, and Macheguenga are from Kaplan (1994), and for the !Kung from Howell (in press). Consumption in NTA project countries includes privately purchased goods based on household surveys and publicly provided in-kind transfers (education, health care, long-term care, etc.), and is averaged across males and females. Labor income includes wages and salaries, fringe benefits, and labor's share of self-employment income, including unpaid family labor (see Lee et al. 2008). For hunter-gatherer groups, labor income is food calories acquired (see Kaplan 1994; Howell, in press). 
(NTA) (see www.NTAccounts.org). This project estimates consumption, labor income, transfers, and asset-based economic activity by age for 30 countries around the world, with complete results currently available for about half of these.

In the hunter-gatherer groups, adults continue to be net producers (negative LCD) until near time of death, transferring the surplus calories to younger group members, presumably mainly relatives. The elderly are estimated to consume about the same or less than other adults. In poor agricultural societies, adults of all ages consume similar amounts, but production falls off at earlier adult ages; so the elderly rely for their consumption on transfers either from the public sector (although outside Latin America public transfers in poor countries are very limited) or from their younger family members, with whom they often live. In contrast to hunter-gatherer societies, in agricultural societies there are typically property rights and property is disproportionately owned by the elderly. When the elderly own the family farm or business, a share of family income accrues to them as property income, even if they work very little. The greatest contrast is with rich industrial societies that have highly developed welfare states. Consumption by the elderly, particularly the very old, is much higher than consumption by young adults, in part because of publicly provided health care and long-term care. Labor income peaks later in the rich countries, depending more on education and training than on physical exertion, but most workers retire early in response to rising demand for

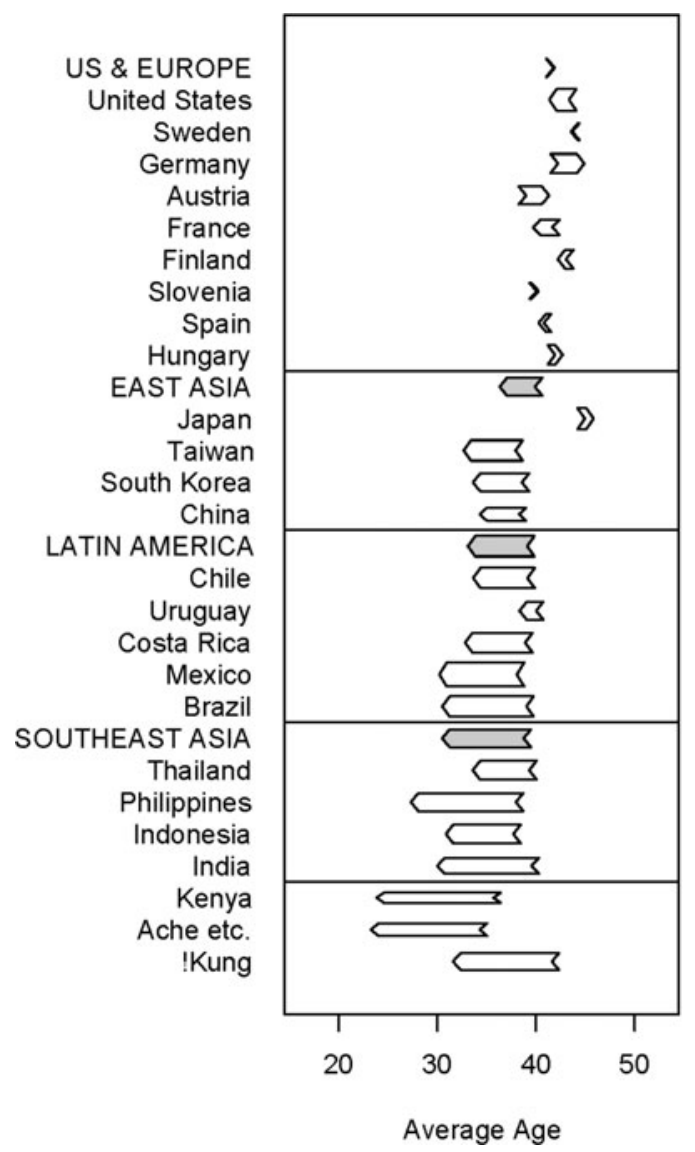

Figure 2 (Lee). Arrow diagrams showing average ages of consumption and labor income in various populations and per capita flows.

The head and tail of the arrow are the weighted average age of consumption or labor income, with the weights supplied by the population times the per capita consumption or labor income profile at each age. Within each region, countries are ordered by per capita GDP, purchasing-parity adjusted. leisure, the availability of pension benefits, and the incentive structure of pensions. Consequently, the old age LCD rises strongly in most rich countries. This reshaped human life cycle, with a long and healthy old age of heavy consumption and little labor effort, is a sea change.

These changes in the economic role of the elderly mean that a potential bonanza of helpful elderly has been transformed instead into economic dependents competing with children for social resources. Throughout history, the net flow of material resources has been downward, from older to younger individuals. Very recently, this direction of net flow has been reversed in some rich countries, and is very likely to be reversed in many more as populations age. Figure 2 illustrates this point by plotting arrows for each society, with the head of the arrow at the average age (population weighted) of consuming and the tail at the average age of producing. The width of each arrow is proportional to the per capita consumption, scaled by average labor income for ages 30 to 49. An arrow pointing to the left indicates resources flowing from older to younger, on average, while an arrow pointing to the right indicates resources flowing on net from younger to older. In hunter-gatherer groups in Kenya, Southeast Asia, Latin America, and East Asia, the regional arrows (shown in gray) and every individual arrow except for Japan point strongly to the left, indicating a net flow of resources to the young. However, in Japan, Hungary, Slovenia, Austria, and Germany, and in the United States and Europe regions as a whole, the arrows now point to the right. This is also a sea change.

Figure 2 combines private transfers, public transfers, and the use of assets to shift consumption. But a similar diagram restricted solely to private transfers shows that these are strongly from older to younger in every one of these societies. It is the public sector transfers that tilt the balance upwards in many rich countries. Furthermore, if we look specifically at transfer patterns of the elderly, we see that in every society outside of East Asia and Thailand, the elderly make private transfers to younger individuals, sometimes out of the public transfers they receive, and sometimes out of their asset income. In addition, the elderly in many countries make substantial bequests, another important route through which resources are transferred to younger generations. The story is complex, but is consistent with a strong motivation of the elderly to assist others privately, even while absorbing massive resources through the public sector.

\section{Measures of grandparental investment as a limiting factor in theoretical and empirical advancement}

doi:10.1017/S0140525X09991701

\author{
Richard L. Michalski \\ Department of Psychology, Hollins University, Roanoke, VA 24020. \\ rmichalski@hollins.edu
}

Abstract: To refine our understanding of grandparental investment and its consequences, we need to understand what grandparents do for their grandchildren. Knowing the landscape of grandparental investment will facilitate a better understanding of the impact of grandparental investment on grandchildren and will allow inroads to be made in bridging the different levels of analysis.

Coall \& Hertwig $(\mathrm{C} \& \mathrm{H})$ call upon researchers to integrate their perspectives to advance our understanding of grandparental investment and its impact on the lives of grandchildren. Clarifying what we mean by grandparental investment may be a worthy step in this endeavor. A complete understanding of the nature of grandparental investment and the effects of grandparental investment on grandchildren will require a comprehensive 
understanding of what such investment is. $\mathrm{C} \& \mathrm{H}$ cite numerous studies looking at the impact of grandparental investment on various outcomes for grandchildren. Most of these studies use measures of grandparental investment that are limited in scope and were likely valued because they were economical to include in large, representative studies. Such measures have outlived their utility. Social distance, time/contact frequency, money, care, and affection represent a sampling of the variables used in the studies cited by C\&H. Grandparental investment, of course, can take many forms, which are highlighted in the target article, but this list is not comprehensive and is inadequate. I will single out one variable.

Spending time with grandchildren can be a useful variable in some studies, but prospecting the behaviors that grandparents engage in while spending time with their grandchildren may prove more valuable in identifying the evolved psychological mechanisms producing these investments. Informing grandchildren about kin relationships and ancestors, offering advice, settling disputes, providing encouragement, showing interest in the activities of grandchildren are just a few of the things that grandparents can do when spending time with grandchildren (Euler \& Michalski 2007). These potentially revealing measures are masked when we use contact frequency as a sole measure of grandparental investment. Time spent with grandchildren in a given period of time is a domain-general measure. Posing domain-general questions thwarts our ability to identify the domain-specific operation of evolved psychological mechanisms adapted for channeling investment to grandchildren. The use of such domain-general measures may be contributing to the difficulty researchers have in crossing interdisciplinary boundaries in order to advance our understanding of grandparental motivations. Contact frequency does not necessarily lend itself to a thorough integration of theoretical perspectives, nor does it inform us about what it is about contact frequency that may be associated with developmental outcomes.

$\mathrm{C} \& \mathrm{H}$ note that there is little to no convincing evidence of sexbiased grandparental investment in humans. This statement may reflect the inadequacy of extant measures of grandparental investment. Grandparents may, for example, channel different investments toward granddaughters than to grandsons, but these potential variables have not yet been empirically documented because of limitations in our tools for assessing grandparental investment. Examining the different types of grandparental investments will allow for more thorough examinations of economic, sociological, anthropological, psychological, and biological discussion.

We also need to exercise care in interpreting reports of grandparental investment. Grandparental, parental, and grandchild reports of grandparental investment may reveal variation attributable to reporting biases. Appreciating the biases that may be prevalent in reports of grandparental investment will help us to better understand how grandparental investment is perceived by kin. In understanding which grandparents invest more than others, for example, Euler et al. (2001) note that responses provided by the recipients of investment may be more accurate, because grandparents may deceive themselves into believing that they do not favor one or a few of their grandchildren.

$\mathrm{C} \& \mathrm{H}$ note the empirical studies documenting biases of grandparents toward grandchildren related through daughters than through sons and related through firstborns and lastborns than through middleborns. This evidence hints at the evolved psychology of parents in mediating grandparental investment and the evolved psychology of grandparents by their responsiveness to parents. A goal of researchers studying grandparental investment might be to better understand the role of parents in encouraging (or discouraging) grandparental investment. What do parents do to encourage grandparents to invest in their grandchildren? Does this encouragement come at the expense of grandparents' investment budgets for grandchildren related through siblings of the parent? To my knowledge, there exists no study examining the ways in which parents attempt to encourage their parents to invest in grandchildren.

The type of investment that grandparents make should matter in the developmental outcomes of grandchildren, and may jumpstart the integration of different theoretical approaches to the study of grandparental investment. Understanding the nature of grandparental investment by means of knowledge of the evolved psychological mechanisms activated in the presence of grandparents, parents, and grandchildren will amplify research efforts and further inform public policy decisions.

\section{The evolutionary versus socio-economic view on grandparenthood: What are the grandparents' underlying motivations?}

doi:10.1017/S0140525X09991713

\author{
Alexander Pashos \\ Institute of Biology, Human Biology and Anthropology, Freie Universität Berlin, \\ 14195 Berlin, Germany. \\ pashos@zedat.fu-berlin.de
}

http://www.biologie.fu-berlin.de/humanbio/alexander-pashos.htm

\begin{abstract}
Coall \& Hertwig $(\mathrm{C} \& \mathrm{H})$ give an ambitious review about the broad range of grandparenting literature from the perspective of different disciplines. They aim to show, how evolutionary theory, sociology, and economics can mutually enrich each other. However, the differences between the evolutionary and the socio-economic perspective should be more clearly pointed out, because they usually deal with different research questions. Grandparents' well-being could be divided into its underlying components.
\end{abstract}

Coall \& Hertwig $(\mathrm{C} \& \mathrm{H})$ comprehensively outline existing theories, results, and interpretations regarding grandparental investment from the perspectives of three different disciplines: evolution, social sciences, and economics. Their overview is careful and diligent, even though occasionally lengthy. A more clear-cut standpoint would be desirable. The authors could take a more pronounced position, instead of trying to accommodate every point of view, especially when certain theoretical differences exist.

Evolutionary versus socio-economic perspectives. The evolutionary biology view and the socio-economic view differ significantly in one point. Evolutionary biology does not assume that grandparents decide rationally when caring for grandchildren. Most behaviors are irrational, although humans have the ability to consider things from a rational point of view.

Human biology searches for universal behaviors and their variety. These behaviors have emerged over the course of evolution because they had an advantage for survival and reproduction. Human behaviors are influenced by emotions and shaped by the social and cultural environment in which we grow up. We learn from an early age what is expected from grandparents and their duties. This means that evolution affects human behavior in terms of both inner motivations and cultural learning. This is because evolution also shapes cultural systems. Grandparental roles must be learned, such as mothering behavior, by observation and experiences. This is not true only for humans, but also for other mammals, not the least being other primates (e.g., Lancaster et al. 1971; Suomi 2003).

Sociology, by contrast, focuses on differences caused by social factors and the economic framework: How is grandparental caregiving characterized within a society, given the socio-economic conditions? And what has changed over time? Biology and sociology, in fact, do not overlap; however, they can complement each other. $\mathrm{C} \& \mathrm{H}$ combine the different interdisciplinary approaches, although they could make different perspectives clearer. When sociologists and economists analyze bidirectional 
intergenerational transfers within a modern Western society or the effects of government transfers, they have their focus on certain conditions. Evolutionary biologists, however, research the universal nature of human behavior, despite the different social-economical parameters.

Asymmetric grandparental care-giving is one such universal pattern that has been found in both sociology and evolutionary science independently of each other. Here, analyses of the underlying proximate mechanisms can be very helpful for an interpretation on an ultimate level. A strong correlation between the parent-grandparent relationship and the grandparents' care of grandchildren has been found (Michalski \& Shackelford 2005; Pashos 2000; Steinbach \& Henke 1998; see also Korchmaros \& Kenny 2001), which can also explain the variance in asymmetric care-giving to a large degree (Pashos \& McBurney 2008). Understanding family relationships is hence not only a sociological topic, but also a key point for an evolutionary interpretation.

The Trivers-Willard hypothesis, or sex-biased grandparental investment, as it is called in the target article, should not be underestimated when explaining male-biased grandparental investment, as found in some traditional patrilineal rural societies (King et al. 2003; Pashos 2000). In patrilateral systems, the investment in sons and grandsons appears to be superimposed upon the universal matrilineality of kin investment.

Significance of grandparents. Grandparental investment developed in evolutionary history because this help was necessary for the offspring's survival. In modern societies, there is an institutional infrastructure that supports parents with their childcare. Is the role of the helping family therefore dispensable nowadays? C\&H correctly conclude that the positive effect of grandparental investment in Western societies should especially be seen with regard to "softer dimensions," such as cognitive and verbal skills during child development and emotional support.

Grandparental investment involves solicitude, listening to problems, telling stories, having daily phone contact, giving protection and support, buying ice cream and toys, getting on one's knees to play with a toddler. These are universal grandparental roles, regardless of the economic conditions in a society. Thus, grandparents are not only "safeguards," "when kids try to set fire to the carpet." The problem is that these so-called softer dimensions, which can benefit child development, are difficult to measure.

Interpretations regarding the transfer of resources form grandparents to children and grandchildren using large socio-economical survey datasets must be done with care. Surveys such as SHARE or the German Aging Survey were not designed for evolutionary approaches. The "regular care" in the SHARE study is defined as "looking after any grandchildren without the presence of the parents." That means that grandchild care can be equated with babysitting here, not with grandparental investment in general. Also, it was not recorded if the grandparents cared for all or only some of their grandchildren. An association of grandparental investment and fertility rates therefore cannot be interpreted with confidence, and is rather questionable.

Significance of grandparenthood for elderly people. Another important problem is the effects of grandparenthood on the elderly people. The authors draw a nonlinear relationship between grandchild care and grandparents' well-being. From an evolutionary theory point of view, the purpose of the mathematical function is not fully clear to me. Investment is always costly. The advantage of kin investment lies in the inclusivefitness benefit. When your child falls into freezing water, and you jump after him, this is, of course, harmful to you. Grandchild care can be exhausting, especially in the "high-risk" context. Surely, it is no full substitute for parental care and the elderly's power is limited. Nevertheless, grandparents are often willing to sacrifice themselves for their grandchildren, more than other kin and non-kin.

But what are their motives? Well-being, life satisfaction, and happiness could be more clearly distinguished. In a data analysis

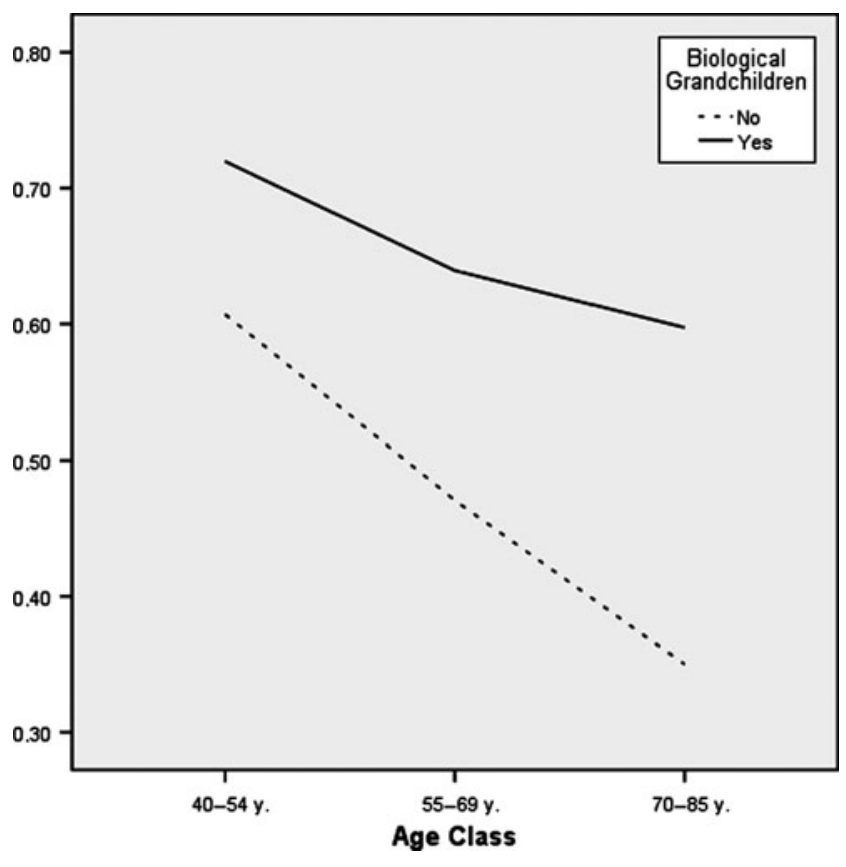

Figure 1 (Pashos). "Are there persons who currently give you great pleasure or great happiness? (Yes $=1$ )" by "Age class" and "Presence of biological grandchildren."

Source: German Aging Study (Tesch-Römer et al. 2002). Available at: http://www.dza.de/nn_12036/SharedDocs/ Publikationen/Alterssurvey_Instrumente,templateId = raw, property $=$ publicationFile.pdf/Alterssurvey_Instrumente.pdf

using the German Aging Survey, I found that the existence of grandchildren, and even of children, does not make elderly people more satisfied with their lives (Pashos 2009). However, grandchildren were very often listed as persons who give great pleasure and happiness. Elderly people without grandchildren were much less able to name persons who gave pleasure and happiness to them, especially with increasing age (Fig. 1). Grandparents also were on average somewhat less lonely than their grandchildless counterparts.

These additional results suggest that the motivation for grandparents to care must not necessarily be looked for in physical and psychological well-being or even the satisfaction with life. Grandparenthood, however, does seem to have an emotional impact on elderly people. It might give them a happy feeling that they are still needed.

\section{The generation game is the cooperation game: The role of grandparents in the timing of reproduction}

doi:10.1017/S0140525X09991725

\author{
Rebecca Sear ${ }^{a}$ and Thomas E. Dickins ${ }^{b}$ \\ a Department of Social Policy, London School of Economics, London WC2A \\ $2 A E$, United Kingdom; ${ }^{\mathrm{b}}$ School of Psychology, University of East London, \\ London E15 4LZ, United Kingdom. \\ r.sear@Ise.ac.uk \\ http://personal.Ise.ac.uk/SEAR/ \\ dickins@uel.ac.uk \\ http://www.uel.ac.uk/psychology/staff/tomdickins.htm
}

Abstract: Coall \& Hertwig $(\mathrm{C} \& \mathrm{H})$ demonstrate the importance of grandparents to children, even in low fertility societies. We suggest 
policy-makers interested in reproductive timing in such contexts should be alerted to the practical applications of this cooperative breeding framework. The presence or absence of a supportive kin network could help explain why some women begin their reproductive careers "too early" or "too late."

We commend Coall \& Hertwig $(\mathrm{C} \& \mathrm{H})$ for addressing an underresearched, but important, issue in the behavioural sciences. Demonstrating that grandparents matter in post-demographic transition, low fertility-low mortality societies is extremely useful. We propose that this cooperative breeding framework may help to explain variation in the timing of reproduction, which is currently of great concern to policy-makers in the developed world. Many developed countries, particularly the United States and the United Kingdom, have strategies in place to reduce teenage pregnancy, seen as detrimental to the mother, child, and society. Equally there is concern about some women "forgetting" to have children, by delaying first births until their fecundity has started to decline.

Relatively early reproduction, including teenage pregnancy, may result partly from a set of circumstances in which kinship networks are still intact and families less dispersed. The proximity and availability of potential grandparents and other close kin may signal to women that early reproduction is feasible and desirable, as this has been a prerequisite for successful reproduction throughout most of our species' history. In contrast, where women leave their kin networks in order to take advantage of education and employment opportunities, they lose these signals from supportive kin that reproduction is likely to be successful, resulting in delayed births.

Within the evolutionary literature, it is becoming accepted that early reproduction is a strategy which makes sense under a particular set of socio-economic circumstances, where young women who have few expectations of being able to increase their human capital through education, for example, make the decision to allocate resources to reproduction (Johns et al, in submission). These decisions clearly happen at a conscious level to some extent (Cater \& Coleman 2006; Lee et al. 2004). However, it is also clear that exposure to specific risks during infancy and childhood also contributes to early fertility in humans. Lack of paternal investment and low birthweight increase the likelihood of a teenage pregnancy markedly (Nettle et al. 2010) and appears to induce more rapid development leading to a smaller adult size and earlier onset of menarche (Nettle et al., in submission). In some populations, it appears that those women who begin their reproductive life sooner also reproduce more (see, e.g., Ministry of Social Development, New Zealand, The Social Report 2009).

C\&H's demonstration that grandparents may be particularly beneficial under conditions of duress, together with this overrepresentation of teenage pregnancy in low socio-economic status (SES) groups, suggests to us that the role of grandparents (i.e., the parents of the teenage mothers) should be conceptualized as a role under harsh conditions. It is possible that the presence of grandparental resources might be a "deciding" factor in early fertility. Second, it would seem that cooperative breeding of this sort may be linked to relatively high fertility rates. This leads us to speculate that the late and low fertility of higher SES women is perhaps a consequence of a shift away from cooperative breeding strategies in which maternal grandparents play an important role.

Women who do choose to invest in their own human capital beneficial in terms of increasing their ability to invest in their children - are less able to rely on a supportive kin network, as they disperse from their families and as their kin, particularly parents, become less able to provide practical support with grandchildren as they age. This results in an increase in the perceived costs of child-raising, necessitating a delay in reproduction until women are in a secure enough financial position to buy in help from non-kin. Such a strategy may also involve relying more on male support, so that additional delay may be introduced by waiting for a suitable partner willing and able to invest in children. This delay may inadvertently result in no reproduction at all.

Such demographic shifts will be accompanied by changes in attitude. Lee et al. (2004) note that in communities with high teenage pregnancy rates, the culture is generally pro-natal and there is often much anti-abortion sentiment. Parents of teenage mothers expose their daughters to a suite of beliefs that encourage reproduction, even in the case of an accidental or unplanned pregnancy. Indeed, Lee et al. (2004) report that the number of abortions in teenage females from low socio-economic backgrounds in the United Kingdom is significantly lower than that for wealthy girls. Different fertility decisions are thus exposed, and the local culture clearly supports these decisions. This leaves open the question as to what wealthier putative grandparents are achieving through their less pro-natal belief systems. One possibility is that by encouraging later, post-higher education fertility, higher SES grandparents-to-be are investing in the quality of their grandchildren, necessary for economic success in a competitive economy.

This also gives the possibility that an alternative explanation for delayed fertility in certain groups of women is not that these women lack the support of their kin for reproduction, but that maternal kin are actively encouraging women to delay until they have invested sufficiently in their own human capital to be able to invest heavily in their offspring. Such grandparents may in fact be investing in their grandchildren, but perhaps in a different currency - financial resources rather than childcare (and a delay in their daughters' reproduction will allow them to accumulate more resources).

Here, we are essentially applying Turke's (1989) and Newson et al.'s (2005) "kin" hypotheses for why modernisation universally results in fertility decline: Modernisation is correlated with a move away from kin-based communities, and greater association with non-kin. Kin help therefore becomes less available and individuals become less exposed to the pro-natalism of relatives, which results in a reduction in fertility. We suggest that similar arguments can be used to explain fertility variation within, as well as between, populations.

$\mathrm{C} \& \mathrm{H}$ end their article by discussing the implications of grandparental investment for fertility. We concur that this should be a priority for future research, and we are beginning such a programme ourselves (Rebecca Sear has recently been involved in a project which demonstrated that British women who have close kin in their social networks have earlier first births than those with looser kin ties; Mathews \& Sear, in preparation). We add that a focus of research should be on the interactions between SES, grandparental investment, paternal investment, and fertility behaviour.

\section{Are humans cooperative breeders?: Most studies of natural fertility populations do not support the grandmother hypothesis}

\author{
doi:10.1017/S0140525X09991749
}

\author{
Beverly I. Strassmann and Nikhil T. Kurapati \\ Department of Anthropology and Research Center for Group Dynamics, \\ University of Michigan, Ann Arbor, MI 48109. \\ bis@umich.edu \\ nikhiltk@umich.edu
}

Abstract: In discussing the effects of grandparents on child survival in natural fertility populations, Coall \& Hertwig $(\mathrm{C} \& \mathrm{H})$ rely extensively on the review by Sear and Mace (2008). We conducted a more detailed 
summary of the same literature and found that the evidence in favor of beneficial associations between grandparenting and child survival is generally weak or absent. The present state of the data on human alloparenting supports a more restricted use of the term "cooperative breeding." Human stem family situations with celibate helpers-at-thenest can be described as cooperatively breeding, but the term is a poor fit to many human family systems.

In the target article, "Grandparental investment: Past, present, and future," Coall \& Hertwig $(\mathrm{C} \& \mathrm{H})$ provide a synthesis of widespread opinion on the evolutionary significance and underpinnings of grandparental nepotism. Their review is thorough and can serve as a useful entrée into the literature for researchers from disparate disciplines. The conjoining of perspectives from evolutionary biology, economics, and sociology is unique.

As first noted by Williams (1957), the long postmenopausal lifespan of women is an evolutionary enigma. Why should natural selection have extended the lifespan beyond the end of fertility? C\&H provide an excellent summary of the main two adaptive hypotheses: the Good Mother Hypothesis and the Grandmother Hypothesis. Here we will comment on the data bearing on grandparental investment in natural fertility populations. A recent review (Sear \& Mace 2008) scored studies with a plus or a minus for whether or not the survival (or presence) of a particular kind of grandparent was associated with improved child survival. Based on this simple dichotomization, Sear and Mace conclude that: (1) "at least one relative is beneficial in almost all populations, suggesting that we are evolved to raise children as an extended family enterprise," (2) "maternal grandmothers tend to improve child survival," and (3) "paternal grandmothers are frequently beneficial but show rather more variation than maternal grandmothers in their effects on child survival" (Sear \& Mace 2008, p. 15). We attempted to replicate this review by looking up the same studies and generating a table that included all findings, together with $p$ values and effect sizes, regardless of whether they were significant (Table 1). We also contacted the authors to request greater specificity (in regard to sample sizes, standard errors, and p-values) and a meta-analysis of the results is in progress (Strassmann and Kurapati, in preparation).

In contrast to the conclusions of Sear and Mace (2008), our assessment of these data is that (1) overall, nonsignificant findings predominate over significant findings, and this is true even in the case of the maternal grandmother; (2) associations between the paternal grandfather and child survival tended to be either nonsignificant or negative; (3) associations for the maternal grandfather were overwhelmingly nonsignificant; and (4) in agreement with Sear and Mace, the situation for the paternal grandmother was extremely variable, but more studies reported positive than negative associations, although many reported nonsignificant associations.

The data are correlational and claims of causation may not be justified, especially in the presence of so many nonsignificant findings. It is also possible that beneficial effects of grandparents on child survival existed that were not discovered by the investigators. Nonetheless, it is unlikely that the weakness of the evidence in favor of grandparenting is entirely on account of problems of study design. The same studies consistently reported strong evidence that maternal survival improves offspring survival (Sear \& Mace 2008), giving these studies some credibility. Most of the children were living in patrilineal, patrilocal populations; negative associations were also found in some matrilineal, matrilocal situations (Sear 2008), but not others (Leonetti et al. 2005). Additional positive associations between grandparernal survival and child survival might have emerged if more matrilocal and foraging societies had been included, but this was not possible to test given the available literature. Our review, like that of Sear and Mace (2008), focused on grandparental survival and grandchild survival, and did not consider other arenas for grandparental nepotism. In conclusion, as shown in Table 1 , the data do not presently support the conclusion that "The presence of a maternal or paternal grandmother was associated with an increase in her grandchildren's probability of surviving in 69\% (9 of 13 studies) and in $53 \%$ (9 of 17 studies) of cases, respectively" (target article, sect. 2.4, first paragraph).

If grandparents, including maternal grandmothers, are less important than has been argued, then the view that humans are a cooperatively breeding species $(\mathrm{C} \& \mathrm{H}$ in the target article; Hrdy 2005b; Kramer 2005a) also requires reassessment. Space does not permit us to consider the evidence for sibling helpers, but it is not stronger than that for grandparents. Child survival and growth is often negatively associated with family size (Lawson \& Mace 2008; Strassmann \& Gillespie 2002), which points to sibling competition rather than cooperation. With these concerns in mind, it is useful to consider the avian literature.

Cockburn (2006) classified bird species as cooperatively breeding if there is evidence that more than $10 \%$ of nests in one or more populations are attended by more than two birds. By this definition, cooperative breeding occurs in $9 \%$ of avian species (Cockburn 2006). Using Cockburn's definition, humans are cooperative breeders if at least two different populations have regular alloparents in at least $10 \%$ of households. This condition is almost certainly met, since two populations out of the total number of human populations is not a high bar to meet. To the best of our knowledge, no one has worked out what proportion of human societies in the past or the present need to have alloparenting (and to what extent) in order for humans to qualify as cooperative breeders. Such a calculation would force us to expose implicit assumptions about the role of grandmothers and other extra-parental helpers.

Rather than using Cockburn's definition, we recommend a more specific and restrictive definition of cooperative breeding when speaking of humans. The helper-at-the-nest phenomenon, wherein grown offspring remain on their natal territory (or farm) and help their siblings to raise nieces and nephews, provides a useful analogy to the pattern of delayed marriage and celibacy among the 19th century rural Irish (Strassmann \& Clarke 1998; see also, Voland et al. 1991 for a German data set). Marriage and celibacy rates in rural Ireland were directly proportional to the availability of farms, and unmarried/non-inheriting siblings often stayed home as helpers, or at least as unpaid laborers. This pattern is comparable to the ecological constraints on independent reproduction in birds (Komdeur 1992; Pruett-Jones \& Lewis 1990; Strassmann \& Clarke 1998).

By restricting the use of the term "cooperative breeding" to situations wherein alloparental behavior is prevalent and direct reproduction is delayed or forfeited, we will be better able to classify and to understand the diversity that exists in human family systems. In behavioral ecology it is more interesting to examine the underlying causes of socioecological or cultural variation than to impose species typical generalizations that may cause us to ignore contrary evidence. At present, the evidence in favor of grandmothering is far weaker than has been generally acknowledged. The significance of the nearly global breakdown of the extended family and the widespread occurrence of autonomous nuclear families, whether monogamous or polygynous, shows that cooperative breeding in humans is facultative. In a wide variety of contexts and countries, responsibility for childcare falls overwhelmingly on the parents. If humans were birds, most societies with nuclear families would not meet the $10 \%$ criterion for alloparenting.

Until the evidence in favor of grandparents and other helpers gets stronger, we suggest that we reserve the term "cooperative breeding" for those societies or family systems that seem to parallel the cooperative breeding found in other species. We should also take a closer look at the "Mother Hypothesis," as the data convincingly show that maternal survival is crucial for offspring survival. 


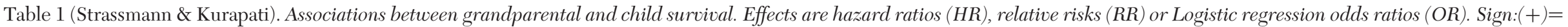

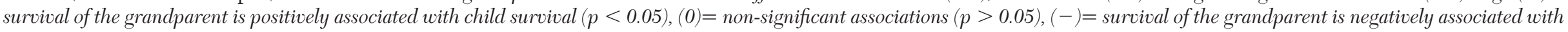
child survival $(p<0.05)$.

\begin{tabular}{|c|c|c|c|c|c|c|c|c|c|c|c|c|c|c|c|c|c|}
\hline \multirow[b]{2}{*}{ Months } & \multirow[b]{2}{*}{ Notes } & \multicolumn{4}{|c|}{ Paternal Grandmother } & \multicolumn{4}{|c|}{ Paternal Grandfather } & \multicolumn{4}{|c|}{ Maternal Grandmother } & \multicolumn{4}{|c|}{ Maternal Grandfather } \\
\hline & & Ratio & $\mathrm{N}$ & $\mathrm{p}$ & Sign & Ratio & $\mathrm{N}$ & $\mathrm{p}$ & Sign & Ratio & $\mathrm{N}$ & $\mathrm{p}$ & Sign & Ratio & $\mathrm{N}$ & $\mathrm{p}$ & Sign \\
\hline \multicolumn{18}{|c|}{ Beise $2005($ ref $=$ dead, RR $)$ - Canada $(\text { Quebec })^{*}$} \\
\hline 0 & boys & 1.13 & 29,431 & $<0.05$ & + & 1.04 & 29,431 & n.s & 0 & 1.06 & 29,431 & n.s & 0 & 1.06 & 29,431 & n.s & 0 \\
\hline 0 & girls & 1.11 & 29,431 & $<0.10$ & 0 & 1.07 & 29,431 & $\mathrm{n} . \mathrm{s}$ & 0 & 1.07 & 29,431 & $\mathrm{n} . \mathrm{s}$ & 0 & 0.95 & 29,431 & n.s & 0 \\
\hline $1-5$ & boys & 0.99 & 29,431 & n.s & 0 & 1.08 & 29,431 & $\mathrm{n} . \mathrm{s}$ & 0 & 1.02 & 29,431 & $\mathrm{n} . \mathrm{s}$ & 0 & 1.03 & 29,431 & $\mathrm{n} . \mathrm{s}$ & 0 \\
\hline $1-5$ & girls & 1.08 & 29,431 & n.s & 0 & 0.99 & 29,431 & n.s & 0 & 1.06 & 29,431 & n.s & 0 & 0.97 & 29,431 & n.s & 0 \\
\hline $6-11$ & boys & 0.95 & 29,431 & $\mathrm{n} . \mathrm{s}$ & 0 & 1.14 & 29,431 & $\mathrm{n} . \mathrm{s}$ & 0 & 1.14 & 29,431 & n.s & 0 & 0.88 & 29,431 & n.s & 0 \\
\hline $6-11$ & girls & 0.97 & 29,431 & $\mathrm{n} . \mathrm{s}$ & 0 & 1.03 & 29,431 & $\mathrm{n} . \mathrm{s}$ & 0 & 0.98 & 29,431 & n.s & 0 & 1.01 & 29,431 & n.s & 0 \\
\hline $12-23$ & boys & 0.94 & 29,431 & n.s & 0 & 1.05 & 29,431 & $\mathrm{n} . \mathrm{s}$ & 0 & 1.33 & 29,431 & $<0.01$ & + & 1.03 & 29,431 & n.s & 0 \\
\hline $12-23$ & girls & 1.12 & 29,431 & $\mathrm{n} . \mathrm{s}$ & 0 & 1.17 & 29,431 & $\mathrm{n} . \mathrm{s}$ & 0 & 1.28 & 29,431 & $<0.01$ & + & 0.99 & 29,431 & n.s & 0 \\
\hline $24-35$ & boys & 0.94 & 29,431 & $\mathrm{n} . \mathrm{s}$ & 0 & 1.14 & 29,431 & $\mathrm{n} . \mathrm{s}$ & 0 & 1.12 & 29,431 & n.s & 0 & 1.01 & 29,431 & n.s & 0 \\
\hline $24-35$ & girls & 1.15 & 29,431 & $\mathrm{n} . \mathrm{s}$ & 0 & 1.07 & 29,431 & $\mathrm{n} . \mathrm{s}$ & 0 & 1.31 & 29,431 & $<0.05$ & + & 1.02 & 29,431 & $\mathrm{n} . \mathrm{s}$ & 0 \\
\hline $36-59$ & boys & 0.98 & 29,431 & $\mathrm{n} . \mathrm{s}$ & 0 & 0.85 & 29,431 & n.s. & 0 & 1.09 & 29,431 & n.s & 0 & 1.29 & 29,431 & $<0.10$ & 0 \\
\hline $36-59$ & girls & 0.91 & 29,431 & n.s. & 0 & 1.51 & 29,431 & $<0.05$ & + & 0.79 & 29,431 & $<0.1$ & 0 & 1.37 & 29,431 & $<0.05$ & + \\
\hline \multicolumn{18}{|c|}{ Borgerhoff Mulder 2007 (ref = dead, HR) - Kenya (Kipsigis) } \\
\hline $0-60$ & & 0.354 & 785 & $<0.01$ & + & 0.600 & 785 & 0.019 & + & 0.740 & 785 & 0.285 & 0 & 1.054 & 785 & 0.832 & 0 \\
\hline \multicolumn{18}{|c|}{ Derosas $2002($ ref $=$ dead, HR $)-$ Italy $(\text { Venice })^{* \ddagger}$} \\
\hline $0-12$ & $\begin{array}{l}\text { both parents } \\
\text { present }\end{array}$ & 0.94 & 11,301 & 0.548 & 0 & 1.20 & 11,301 & 0.090 & 0 & 0.98 & 11,301 & 0.779 & 0 & 0.92 & 11,301 & 0.320 & 0 \\
\hline $0-12$ & fatherless & 0.63 & 11,301 & 0.051 & 0 & 0.89 & 11,301 & 0.602 & 0 & 0.89 & 11,301 & 0.096 & 0 & 1.05 & 11,301 & 0.796 & 0 \\
\hline $24-120$ & $\begin{array}{l}\text { both parents } \\
\text { present }\end{array}$ & 1.06 & 11,301 & 0.734 & 0 & 0.97 & 11,301 & 0.897 & 0 & 1.16 & 11,301 & 0.321 & 0 & 0.89 & 11,301 & 0.487 & 0 \\
\hline $24-120$ & fatherless & 1.10 & 11,301 & 0.832 & 0 & 0.48 & 11,301 & 0.103 & 0 & 0.84 & 11,301 & 0.632 & 0 & 0.75 & 11,301 & 0.439 & 0 \\
\hline $24-120$ & motherless & 0.13 & 11,301 & 0.068 & 0 & 0.65 & 11,301 & 0.645 & 0 & 0.27 & 11,301 & 0.107 & 0 & 1.36 & 11,301 & 0.721 & 0 \\
\hline \multicolumn{18}{|c|}{ Gibson \& Mace $2005($ ref $=$ dead, OR $)-$ Ethiopia $($ Oromo $)$} \\
\hline $0-60$ & girls & 0.674 & 1,144 & $<0.05$ & + & 1.037 & 1,044 & n.s. & 0 & 0.940 & 1,435 & n.s. & 0 & 0.763 & 1,435 & n.s. & 0 \\
\hline $0-60$ & boys & 1.096 & 1,187 & n.s. & 0 & 0.841 & 1,073 & n.s. & 0 & 0.714 & 1,474 & n.s. & 0 & 0.889 & 1,473 & n.s. & 0 \\
\hline \multicolumn{18}{|c|}{ Hill and Hurtado 1996 (ref = dead, OR, maternal and paternal grandparents are lumped together) - Paraguay (Ache) } \\
\hline $0-48$ & reservation & 0.7234 & Unclear & 0.320 & 0 & 1.193 & unclear & 0.510 & 0 & 0.7234 & unclear & 0.320 & 0 & 1.193 & unclear & 0.510 & 0 \\
\hline $0-108$ & forest period & 0.8424 & Unclear & 0.240 & 0 & 0.8249 & unclear & 0.156 & 0 & 0.8424 & unclear & 0.240 & 0 & 0.8249 & unclear & 0.156 & 0 \\
\hline \multicolumn{18}{|c|}{ Jamison et al. $2002($ ref $=$ absent, OR $)-$ Japan $(\text { Central })^{\ddagger}$} \\
\hline $0-192$ & girls & 0.834 & 8617 & 0.132 & 0 & 1.440 & 8617 & 0.045 & - & 0.884 & 8617 & 0.367 & 0 & 1.246 & 8617 & 0.669 & 0 \\
\hline $0-192$ & boys & 1.384 & 9854 & 0.014 & - & 0.996 & 9854 & 0.982 & 0 & 0.480 & 9854 & 0.060 & 0 & 1.263 & 9854 & 0.651 & 0 \\
\hline \multicolumn{18}{|c|}{ Kemkes-Grottenthaler 2005 (ref = dead, RR) - Germany (Ludwigshafen)* } \\
\hline 0 & & 1.489 & 1,590 & 0.025 & + & 0.631 & 1,590 & 0.011 & - & 1.034 & 1,590 & 0.852 & 0 & 1.105 & 1,590 & 0.573 & 0 \\
\hline 6 & & 1.406 & 1,590 & 0.003 & + & 0.653 & 1,590 & 0.003 & - & 1.084 & 1,590 & 0.567 & 0 & 0.957 & 1,590 & 0.753 & 0 \\
\hline 12 & & 1.328 & 1,590 & 0.029 & + & 0.684 & 1,590 & 0.004 & - & 0.969 & 1,590 & 0.809 & 0 & 1.140 & 1,590 & 0.309 & 0 \\
\hline
\end{tabular}


Table 1 (Strassmann \& Kurapati) (Continued)

\begin{tabular}{|c|c|c|c|c|c|c|c|c|c|c|c|c|c|c|c|c|c|}
\hline \multirow[b]{2}{*}{ Months } & \multirow[b]{2}{*}{ Notes } & \multicolumn{4}{|c|}{ Paternal Grandmother } & \multicolumn{4}{|c|}{ Paternal Grandfather } & \multicolumn{4}{|c|}{ Maternal Grandmother } & \multicolumn{4}{|c|}{ Maternal Grandfather } \\
\hline & & Ratio & $\mathrm{N}$ & $\mathrm{p}$ & Sign & Ratio & $\mathrm{N}$ & $\mathrm{p}$ & Sign & Ratio & $\mathrm{N}$ & $\mathrm{p}$ & Sign & Ratio & $\mathrm{N}$ & $\mathrm{p}$ & Sign \\
\hline 18 & & 1.214 & 1,590 & 0.117 & 0 & 0.741 & 1,590 & 0.017 & - & 1.029 & 1,590 & 0.820 & 0 & 1.058 & 1,590 & 0.648 & 0 \\
\hline 24 & & 1.156 & 1,590 & 0.043 & + & 0.777 & 1,590 & 0.043 & - & 0.907 & 1,590 & 0.428 & 0 & 1.116 & 1,590 & 0.362 & 0 \\
\hline \multicolumn{18}{|c|}{ Sear et al. 2000 ( ref = alive (non-reproductive alive for maternal grandmother), OR) - Gambia (four villages) } \\
\hline $0-11$ & & 0.80 & 765 & n.s. & 0 & 1.06 & 536 & n.s. & 0 & 1.16 & 1,083 & n.s. & 0 & 0.91 & 826 & n.s. & 0 \\
\hline $12-23$ & & 0.85 & 765 & n.s. & 0 & 0.71 & 536 & n.s. & 0 & 2.04 & 1,083 & $<0.05$ & + & 0.99 & 826 & n.s. & 0 \\
\hline $24-59$ & & 1.07 & 765 & n.s. & 0 & 0.84 & 536 & n.s. & 0 & 1.02 & 1,083 & n.s. & 0 & 1.07 & 826 & n.s. & 0 \\
\hline \multicolumn{18}{|c|}{ Sear et al. 2002 (ref = alive, OR $)$ - Gambia (four villages $)$} \\
\hline $0-11$ & & 0.78 & 2,294 & n.s. & 0 & 1.32 & 2,294 & n.s. & 0 & 1.14 & 2,294 & n.s. & 0 & 1.07 & 2,294 & n.s. & 0 \\
\hline $12-23$ & & 0.84 & 1,664 & n.s. & 0 & 0.95 & 1,664 & n.s. & 0 & 1.74 & 1,664 & n.s. & 0 & 1.32 & 1,664 & n.s. & 0 \\
\hline $24-59$ & & 0.95 & 1,341 & n.s. & 0 & 0.75 & 1,341 & n.s. & 0 & 0.91 & 1,341 & n.s. & 0 & 1.01 & 1,341 & n.s. & 0 \\
\hline \multicolumn{18}{|c|}{ Sear $2008($ ref $=$ alive, OR, mm $)-$ Malawi $($ Chewa $)$} \\
\hline $0-60$ & dead & 1.06 & 1,128 & n.s. & 0 & 1.36 & 1,144 & n.s & 0 & 0.47 & 1,633 & $<0.05$ & - & 1.20 & 1,635 & n.s. & 0 \\
\hline $0-60$ & absent & 2.74 & 1,128 & $<0.01$ & + & & & & & 1.03 & 1,128 & n.s. & 0 & & & & \\
\hline \multicolumn{18}{|c|}{ Strassmann et al. in prep $($ ref $=$ dead, HR $)-$ Mali $($ Dogon $)$} \\
\hline $1-60$ & & 2.022 & 5,583 & $<0.01$ & - & 1.28 & 5,583 & 0.301 & 0 & 0.92 & 5,583 & 0.707 & & 1.13 & 5,583 & 0.507 & 0 \\
\hline \multicolumn{18}{|c|}{ Tymicki 2006 (ref = alive, RR) - Poland (Bejsce parsish) } \\
\hline $0-12$ & & 1.24 & 3,587 & $\leq 0.01$ & + & 1.38 & 3,587 & $\leq 0.01$ & + & 1.20 & 3,587 & $\leq 0.01$ & + & 1.49 & 3,587 & $\leq 0.01$ & + \\
\hline $0-60$ & & 1.14 & 6,785 & $\leq 0.01$ & + & 1.22 & 6,785 & $\leq 0.10$ & 0 & 1.08 & 6,785 & $\leq 0.10$ & 0 & 1.31 & 6,785 & $\leq 0.01$ & + \\
\hline
\end{tabular}

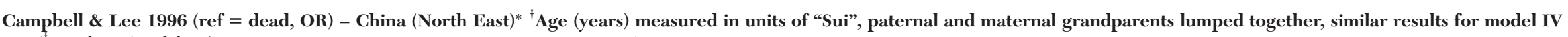

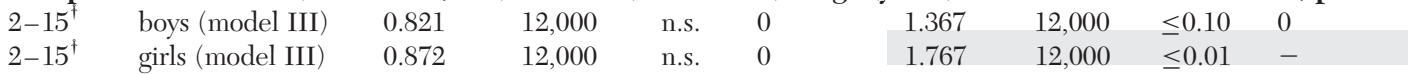

Griffiths et al. $2001($ ref $=$ present, HR $)-$ India

$\begin{array}{llrrrr}0-1 & \text { Maharashtra } & 2.11 & \text { Unclear } & \text { n.s } & 0 \\ 0-1 & \text { Tamil Nadu } & 2.41 & \text { Unclear } & \text { n.s. } & 0 \\ 0-1 & \text { Uttar Pradesh } & 2.13 & \text { Unclear } & \leq 0.01 & + \\ 1-8 & \text { Uttar Pradesh } & 0.17 & \text { Unclear } & \text { n.s. } & 0 \\ 9-23 & \text { Uttar Pradesh } & 0.16 & \text { Unclear } & \text { n.s. } & 0\end{array}$

Lahdenperä et al. $2004\left(\right.$ ref $=$ dead, log-rank test $\left.\mathrm{X}_{2}{ }^{2}\right)-$ Finland $-18^{\text {th }} \& 19^{\text {th }}$ centuries

0-180

Ladusing and Holendro Singh 2006 (ref = absent) - India (North East)

$\begin{array}{llll}0-60 & 0.84 & 7,774 & \text { n.s. }\end{array}$

Leonetti et al. $2005($ ref $=$ alive, $\mathrm{HR})-$ India $($ Khasi $)$

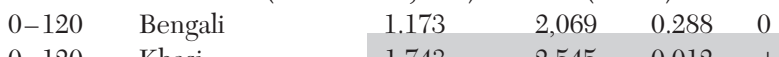

$\begin{array}{lllll}0-120 & \text { Khasi } & 1.743 & 2,545 & 0.012\end{array}$ 
Commentary/Coall \& Hertwig: Grandparental investment

\title{
Evolutionary psychology's notion of differential grandparental investment and the Dodo Bird Phenomenon: Not everyone can be right
}

doi:10.1017/S0140525X09991737

\author{
Martin Voracek, ${ }^{a}$ Ulrich S. Tran, ${ }^{\mathrm{b}}$ and Maryanne L. Fisher ${ }^{\mathrm{c}}$ \\ ${ }^{a}$ Department of Basic Psychological Research, School of Psychology, \\ University of Vienna, A-1010 Vienna, Austria; ${ }^{\mathrm{b}}$ Department of Clinical, \\ Biological, and Differential Psychology, School of Psychology, University of \\ Vienna, A-1010 Vienna, Austria; ' Department of Psychology, St. Mary's \\ University, Halifax, Nova Scotia, $\mathrm{B} 3 \mathrm{H} 3 \mathrm{C} 3$, Canada. \\ martin.voracek@univie.ac.at \\ http://homepage.univie.ac.at/martin.voracek/ \\ ulrich.tran@univie.ac.at \\ mlfisher@smu.ca
}

000000

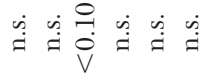

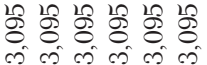

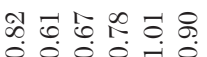

Abstract: Integration of different lines of research concerning grandparental investment appears to be both promising and necessary. However, it must stop short when confronted with incommensurate arguments and hypotheses, either within or between disciplines. Further, some hypotheses have less plausibility and veridicality than others. This point is illustrated with results that conflict previous conclusions from evolutionary psychology about differential grandparental investment.

We congratulate Coall \& Hertwig $(\mathrm{C} \& \mathrm{H})$ for their outstanding contribution. It certainly will have a noticeable impact on the respective research fields addressed therein. This work is a paramount example of the far too rarely occurring theoretical integration of work on one specific topic (here, grandparental investment) that is investigated across different disciplines and from distinct perspectives. This diversity of viewpoints is true for grandparental investment, which is studied in sociology, economics, as well as through the lenses of evolutionary theory, as applied in evolutionary psychology.

As $\mathrm{C} \& \mathrm{H}$ rightly conclude, significant parts of these literatures are distinct and at times disjointed and contradictory; are not well integrated; and have few discernible tendencies for fruitful exchange amongst themselves. Although we appreciate the general thrust of C\&H's arguments, which calls for a broad integration of different viewpoints, we feel this might go too far and may well be "overly friendly" with respect to recent disparate views regarding the nature of grandparental investment. Simply put, opposing views and competing explanations of which one would rule out the opposite argument are unlikely to be equally plausible and hence both cannot be "true."

This is, to some extent, reminiscent of past views (now outdated and quite clearly rebutted) in an entirely different research field, namely psychotherapy research. In the 1970s, this field became entrenched by opinions that now are encompassed by the "Dodo Bird Phenomenon" (alluding to the dodo figure appearing in Lewis Carroll's novel Alice's Adventures in Wonderland). Specifically, we now know that differential psychotherapy research (i.e., efficiency and efficacy studies comparing different psychotherapeutic schools, approaches, and techniques) erroneously concluded that, like in Carroll's novel, "everyone has won and all must have prizes" (cf. Luborsky et al. 1975). These views have subsequently been quite clearly rebutted (e.g., Beutler 1991; Shadish \& Sweeney 1991). With regard to opposing evidence about grandparental investment from the evolutionary, sociological, and economic literatures, there appears to be the potential danger of a similar "Dodo Bird Phenomenon."

There is mounting evidence which suggests that important conclusions from the currently widespread evolutionary psychological reasoning about differential grandparental investment and solicitude might not be veridical, but rather be a result of neglected effects of confounding variables. For example, age and residential distance were found to be associated with investment and solicitude ratings, but were not statistically controlled in the studies of Euler and Weitzel (1996), Steinbach and Henke 
(1998), and Pashos (2000). Specifically, evolutionary psychological research has introduced the notion that laterality effects are important in grandparental investment. There exist two distinct types of grandparents (matrilateral vs. patrilateral ones), which are distinguished by different degrees of paternity certainty, and thus, potentially different degrees of genetic relatedness (see target article, sect. 5.1.3.). Indeed, many studies conducted in modern industrialized nations (see target article, top of sect. 2. and Note 4) have obtained a specific pattern in the ranking of grandparental investment, as recalled by adults using rating scales, such that the maternal grandmother seemed to care the most, the maternal grandfather somewhat less, followed by the paternal grandmother, and then the paternal grandfather.

Elsewhere (Tran et al. 2009), we show that the well-known spousal age differences, combined with the equally pervasive sex differences in life expectancy, create noticeable differences in the expected exposure time grandchildren have to their four grandparents. That is, due to the fact that grooms are generally older and become parents later in life than do brides, grandchildren have a shorter overlap in the number of years they could interact with grandfathers than they do with grandmothers.

It is important to note that the ranking of expected exposure time to the four grandparents follows exactly the abovementioned ranking of recalled grandparental solicitude. Clearly, total grandparental investment in grandchildren must be limited by (and thus partly dependent on) the available time that grandparents have for such investments in their grandchildren. Intriguingly, appropriate controls for exposure time, accounting for the two confounding variables noted earlier, significantly level the matrilateral bias in grandparental investment (Tran et al. 2009). We therefore conclude that the widely publicized matrilaterality effect of grandparental investment, as highlighted in current evolutionary psychological literature, may well be incorrect, or at least substantially overstated.

This finding has manifold ramifications and consequences. As for one example, some researchers (e.g., Gaulin et al. 1997; Russell \& Wells 1987) have used such retrospective, obviously biased rating data of adults' recalled grandparental solicitude for estimating nonpaternity rates. Unsurprisingly, such calculations of putative nonpaternity rates have yielded grossly overstated and thus implausible values $(10-20 \%)$ - which clearly indicates the inappropriateness of such rating data. In contrast, several recent systematic reviews of nonpaternity rates among modern human populations have convergently estimated these to be about $2-3 \%$ (Anderson 2006; Bellis et al. 2005; Voracek et al. 2008).

Of further importance in this context, in their systematic review of grandparental investment and physical and mental well-being of grandchildren (sect. 5.1.), C\&H cite studies (sect. 5.1.2.) that found differential grandparental investment (i.e., the matrilateral bias therein) to be dissociated from the criterion variables under scrutiny. This constitutes more counterevidence to current evolutionary psychological reasoning about the existence, importance, and consequences of differential grandparental investment, as the allegedly differentiated pattern of grandparental caring showed a lack of association with important life outcomes in the grandchildren.

Having taken evolutionary psychological study findings as examples for illustration, we therefore would suggest that the opposed views of sociology, economics, and evolutionary psychology on grandparental investment appear hard to reconcile and to integrate, as intended and anticipated by $\mathrm{C} \& \mathrm{H}$. There may well be no "Dodo Bird Phenomenon" in these lines of research, and we do not believe that "everyone has won and all must have prizes." At the very least, some of these competing hypotheses have less plausibility and veridicality than others. Specifically, we surmise that some important explanations and assertions in this field based on current evolutionary psychology thinking might be confounded or misleading, as they could be more parsimoniously derived from quite simple facts of demography, as we have elaborated here.

\section{Authors' Response}

\section{Toward an integrative framework of grandparental investment}

\author{
doi:10.1017/S0140525X10000014
}

\author{
David A. Coall ${ }^{a}$ and Ralph Hertwig ${ }^{b}$ \\ a School of Psychiatry and Clinical Neurosciences, University of Western \\ Australia, Fremantle, Western Australia 6160, Australia; ${ }^{\mathrm{b}}$ Department of \\ Psychology, University of Basel, 4055 Basel, Switzerland. \\ david.coall@uwa.edu.au \\ http://www.uwa.edu.au/people/david.coall \\ ralph.hertwig@unibas.ch \\ http://www.psycho.unibas.ch/hertwig
}

Abstract: This response outlines more reasons why we need the integrative framework of grandparental investments and intergenerational transfers that we advocated in the target article. We discusses obstacles - from misconceptions to poor measures - that stand in the way of such a framework and of a better understanding of the effects of grandparenting in the developed world. We highlight new research directions that have emerged from the commentaries, and we end by discussing some of the things in our target article about which we may have been wrong.

\section{R1. Introduction}

One main goal of our target article was to review the existing disparate accounts of grandparental investment and intergenerational transfers, and to make a case for an integrative framework that promotes consilience among psychological, evolutionary, economic, and sociological accounts. Taking steps toward a multidisciplinary framework is not easy, because the involved disciplines have different goals, terminologies, assumptions, methods, and to some extent conflicting worldviews. Therefore, we are pleased that 18 commentators from the fields of anthropology, demography, economics, evolutionary biology, evolutionary and developmental psychology, and sociology responded to our article. We thank the authors for their generous comments, valuable insights, and helpful suggestions. We are especially grateful to those contributors who extended our understanding of research on grandparental investment in new directions (see sect. R6). In our response, we first turn to our commentators' views concerning our vision of an integrative and multidisciplinary framework. Throughout our reply, we also attend to our omissions, errors, and confusions, where appropriate, as an antidote to the risk of simplifying too much in our attempt to bring together disparate accounts. Unfortunately, some good points made by individual commentators - especially those with which we agree and therefore feel less impelled to comment on - must go unaddressed.

\section{R2. Grandparental investment: Theory pluralism and theory integration}

Many commentators appear to agree with theoretical pluralism and support our push for a multidisciplinary framework in research on grandparental investment; some say so explicitly (Fantino \& Stolarz-Fantino; Huber; 
Voracek, Tran, \& Fisher [Voracek et al.]). Such an integrative framework, however, does not mean that we have to believe that each hypothesis has equal explanatory weight (a position that Voracek et al. appear to attribute to us). Of course, the evidence should be allowed to sort the wheat from the chaff. In this section, we clarify some reasons for the need of a multidisciplinary framework (R2.1), consider overlooked aspects that need to be included (R2.2), and address concerns regarding the practicality of a multidisciplinary framework (R2.3).

\section{R2.1. Why we need a multidisciplinary framework}

A multidisciplinary framework is not l'art pour l'art. Grandparental investments in industrialized societies are part of multilayered intergenerational transfers, both private and public. Consequently, what happens within families cannot be divorced from public transfers via institutions created by the social welfare state. Therefore, we are convinced that economic and sociological analyses of, for example, public transfers need to be integrated with analyses of private transfers. Moreover, both kinds of transfers need to be combined with demographic analyses of fertility and mortality, and with psychological analyses of the health and well-being consequences for both the recipients and the donors. No single discipline can do it all by itself.

Lee and Cox bring up the topic of public intergenerational transfers. In his compelling analysis, Lee shows that in rich industrialized societies, consumption by older adults (age 60 and above) - for example, in terms of health care, long-term care, and pensions - is much higher than consumption by younger adults. In addition, older adults' consumption exceeds their production (measured as labor earnings), a condition that in hunter-gatherer societies is characteristic of childhood only. According to Lee, this represents a paradigm shift in human history, with the net flow of material (in vivo) resources being reversed from downward to upward and amounting to a relatively brief period of 30 years during which adult Americans, for example, produce more than they consume (Lee et al. 2006). As Lee stresses, however, this reversal has occurred for public, but not private, material transfers (see also, Kohli 1999).

Lee's analysis raises the question of how public and private transfers relate to each other: Do they complement or antagonize each other? Cox touches upon a concept discussed by economists and sociologists that is key in answering this question: crowding out. Used in the context of intergenerational transfers, crowding out describes the displacement of private help within the family by public, institutionalized services. Various authors have suspected crowding out as the cause behind the weakening of intergenerational links within the family (see the discussion in Künemund \& Rein 1999). With the expansion of the welfare state, crowding out may occur for a wide range of private transfers, downward and upward alike. Focusing on upward transfers, Brandt et al. (2009) analyzed the interaction of private and public aid in the context of support that children give to their elderly, ailing parents (an issue to which we return in sect. R6.2). Based on the SHARE (Survey of Health, Ageing and Retirement in Europe) data set, they analyzed the level of care that adult children gave a parent during the previous 12 months in 11 European countries. Their main finding was a division of labor between private and public help: Specifically, professional services have taken over the medically more demanding and physical help of dependent recipients (thus crowding out private activities). The adult children, in turn, tend to provide voluntary, and less intensive support (e.g., help in the household or in dealing with authorities). On Brandt et al.'s interpretation, public support thus does not generally displace private support, but the services are divided, and the availability of a public support system might actually stimulate ("crowd in") private support. When social and health services, however, are less available - as is the case in the Mediterranean, relative to Scandinavian countries - children are more likely to provide the intensive care that ailing parents require. Such demanding care, however, appears to exact a cost: It has been suggested that simultaneous care for elderly parents and young children may overtax families and contribute to the low fertility rate in Mediterranean countries (Livi-Bacci 2001), which provide less public and institutional support for both the raising of children and the care for elderly, ailing parents.

To the best of our knowledge, such an analysis of the interplay between private and public transfers and their impact on the dimensions of interest, including classic fitness indicators (e.g., fertility, childhood mortality, age at first reproduction) and psychological and economic indicators of health and well-being, has not yet been conducted with regard to grandparental resources in the developed world. Does public support - in terms of child support, daycare facilities, and free education crowd out private transfers from grandparents to grandchildren (and their parents)? Alternatively, has a division of labor evolved, with time-intensive and demanding services being provided by public institutions (if available), allowing grandparents to step up other investments? Relatedly, to what extent do private transfers from grandparents to grandchildren depend on public transfers such as institutionalized pension systems: In other words, what parts of the public transfers from employed adult children to retired grandparents are handed back to them via grandchildren (see also, Kohli 1999)?

At the risk of sounding repetitive: In modern societies, private grandparental investments are intertwined with other intergenerational transfers. Investigating the consequences of these transfers on psychological, economic, demographic, sociological, and medical dimensions and examining the motivations underlying private, non-institutionalized transfers requires, so we believe, theory pluralism and theory integration.

\section{R2.2. Why we need lifespan developmental psychology}

Hoppmann \& Klumb remind us gently of an additional perspective on grandparental investment that needs to be part of an integrative framework, namely, lifespan developmental psychology (see also, Gurven \& Schniter). We wholeheartedly agree, and would also advocate a similar approach in sociology, the study of the life course. Key to the lifespan development perspective is the assumption that ontogenetic development extends 
across all stages of the life course, from conception to death. One way to conceptualize development is in terms of demands and opportunities - or Havighurst's (1948/1972) notion of developmental tasks - that individuals face as they move through life. One developmental task associated with parenting and grandparenting is, couched in Erikson's (1966) classic (and somewhat rigid) stage model of psychosocial development, the solution of the conflict between generativity and stagnation. In our view, early and modern theories of lifespan development psychology - for instance, the selection, optimization, and compensation framework (Baltes 1997), or the socioemotional-selectivity theory (Carstensen et al. 1999) can bring three things to an integrative framework of grandparental altruism: First, they can broaden our understanding of why grandparents care for younger generations. Second, they can offer hypotheses as to why such activities can have tangible benefits for grandparents in terms of a sense of identity and accomplishments (an issue to which we return in sect. R6.1), and successful aging. Third, they will be indispensible in understanding how investments translate into psychological outcomes, in both grandchildren and grandparents. To this end, we hope that life-span development psychologists will discover that grandparenting - a topic they have largely neglected so far - is worth their attention.

\section{R2.3. Necessary scrutiny and unnecessary misconceptions}

Voracek et al. criticize us for being too accommodating of the different theoretical perspectives and thus overlooking serious flaws in one or the other approach. According to them, one potential flaw in the evolutionary literature is the often-found pattern of grandparental investment according to which the maternal grandmother invests the most, followed by the maternal grandfather, the paternal grandmother, and the paternal grandfather (see sect. 2.3 in our target article). Voracek et al. criticize evolutionary investigations reporting this pattern for not adjusting for potential confounding factors such as grandparental age and residential distance. Both might account for the apparently robust association between grandparental type and grandparental investment. We do, of course, agree that establishing the independent effect of grandparent type by adjusting for potential confounders, such as grandparental age, health, wealth, and distance, is good practice. However, the seminal article reporting this pattern and cited by Voracek et al. as a culprit (Euler \& Weitzel 1996) recognized, investigated, and found no evidence for a confounding role of grandparental age and distance (pp. 46-48).

Irrespective of the merits of the conjecture that evolutionary investigations fail to control for likely confounders - one can find examples of better (e.g., Coall et al. 2009; Pollet et al. 2009) and worse practice (Bishop et al. 2009) - we have not advocated an "anything goes" approach. Pursuing an integrative framework does not mean that we have to believe that each hypothesis has the same merit and should not be subjected to empirical scrutiny. In fact, having one's favorite hypothesis inspected by researchers from other disciplines, with different theoretical priors and methods, will make the hypothesis' merits, or lack thereof, more compelling.
Joining forces toward building a multidisciplinary framework sounds fine in theory, but can it work in practice? Some express doubts. The evolutionary psychologist Euler diagnoses among sociologists a "resistance to biological accounts of human behavior," manifest in their lack of interest in lineage and gender effects. When read side-by-side, Euler's and Friedman \& Hechter's commentaries show that the breakdown of communication across disciplines in research on grandparental investment is most palpable between evolutionary psychology and sociology. This breakdown makes it possible that to one degree or another we all hold outdated misunderstandings of the other discipline. Take the conjecture that sociologists rarely examine grandparental sex and lineage in their analyses. Although it may have been accurate in the past, things are changing (e.g., Chan \& Elder 2000; Dubas 2001; Monserud 2008).

Change would be ushered in even faster if we began to read and cite the other discipline's work in earnest. In a recent citation analysis, we (Coall \& Hertwig, submitted) found mostly mutual neglect. Analyzing the reception of three seminal articles authored by evolutionary researchers (Daly \& Wilson 1980; Euler \& Weitzel 1996; Hawkes et al. 1998) and sociological researchers (Bengtson 2001; Bengtson \& Roberts 1991; Szinovacz 1998a), respectively, all of which explored intergenerational transfers and relationships, we found that although they had been cited a total of 644 times, only on 22 occasions (3.4\%) were they cited by the other discipline. This disinterest in each other is most striking because the methodologies of each discipline, specifically in the areas of discriminative grandparental solicitude (Euler \& Weitzel 1996; Michalski \& Shackelford 2005) and intergenerational solidarity (e.g., Mangen et al. 1988), are becoming more similar. Moreover, the methodological rigor of sociology is likely to benefit evolutionary investigations, and the theoretical framework of evolutionary theory may widen the scope of hypotheses examined by sociologists. It is time to stop pointing fingers, and to start benefiting from each other.

Not surprisingly, the commentators most critical of our target article were those of whose theory of grandparental investment (Friedman et al. 2008) we were quite skeptical (see sect. 3.2 in our target article). We appreciate Friedman \& Hechter's commentary in which they put a number of misconceptions of the evolutionary view of grandparental investment out into the open. We cannot address all of these but focus on a few, and hope that their discussion can help in moving beyond them. Contrary to Friedman \& Hechter's implication that massive investments into post-pubertal children in developed societies cannot be explained within an evolutionary framework, let us point out that a quantity-quality tradeoff is a key concept within evolutionary, demographic, and economic accounts (see e.g., Kaptijn et al. 2010; Van Bavel 2006). Specifically, in post-demographic transition societies, ensuring that descendants are competitive in employment and mating markets means that investment extends way beyond puberty and often into the children's own parenthood. Such investments in the high within-population competitiveness of modern societies exact opportunity costs and consequences for the investor's inclusive fitness, and, therefore, conform to Trivers' (1972) definition of parental investment. 
Relatedly, Friedman \& Hechter also conjecture that there is no satisfactory evolutionary explanation for the declining fertility in developed countries. Indeed, sociologists have previously identified the demographic transition as a challenge to evolutionary perspectives (e.g., Vining 1986). In our view, this challenge has been met by theoretical reviews detailing evolutionary hypotheses (e.g., Borgerhoff Mulder 1998) and by combining evolutionary and demographic expertise (e.g., Clarke \& Low 2001). Currently, a combination of hypotheses gives the strongest evolutionary account of the demographic transition (see Sear \& Dickins). This is not surprising because the demographic transition takes different forms in response to the complexity of different ecological and social conditions (e.g., Kaptijn et al. 2010). We concede that even though a combination of hypotheses may give a more realistic representation of the complex social changes taking place, it may simultaneously leave the unfortunate impression that no satisfactory evolutionary explanation exists.

Finally, Friedman \& Hechter ask why grandparents do not limit their investment into their children and simply assume that their children will do the same, given that their objective, according to Friedman \& Hechter's reading of the "evolutionary model," is to maximize the well-being of their children. There are different answers to this question. Let us give two. First, an important basic distinction is that between classic and inclusive fitness. Introduced by Hamilton (1964), inclusive fitness emphasizes that an evolutionary perspective is not purely about ensuring your own reproduction. Inclusive fitness is calculated from an individual's own reproductive success (classic fitness) in combination with his effects on the reproductive success of his relatives, each one weighted by the coefficient of relatedness. That is, individuals can get proportions of their genes into future generations by ensuring individuals who share some of their genes by common descent survive and reproduce. This can include children and grandchildren, but also nieces, nephews, and more distant relatives. Maximizing inclusive fitness is thus one possible explanation for why grandparents invest in kin other than their children (as detailed in terms of the grandmother hypothesis in sect. 1.2 of our target article). Another explanation for grandparental altruism that is also consistent with an evolutionary framework is in terms of evolutionary continuity and the empathy mechanism (see sect 3.3 in our target article).

Pashos also harbors some misconceptions, in this case of his own field: evolutionary psychology. We are puzzled by his pronouncement that "most behaviors are irrational," leaving it open as to what notion of rationality he may have in mind. He also draws a strict distinction between evolutionary and economic accounts of human behavior, suggesting that evolutionary biologists discover human regularities devoid of economic conditions. We disagree. Of course, economic and evolutionary concerns are related, and in contrast to Pashos' view, yes, evolutionary theorists do talk about economic and, more generally, ecological factors. Life history theory, a branch of evolutionary ecology, is effectively evolutionary economics: the allocation of limited resources among competing demands during a finite lifetime for the greatest utility (in this case, reproductive success). Examples of evolutionary-minded researchers describing regularities of human behavior in response to economic conditions are endless (see, e.g., Clarke \& Lowe 2001). To name a few such regularities: In traditional and historical societies, marriage rates and fertility patterns have been found to correlate with resource fluctuations. In most societies, men have been demonstrated to use resources such as wealth and status to gain reproductive advantages. Child survival varies with socioeconomic status of the family, and child abandonment is related, among other factors, to economic conditions. Biased investment favoring daughters over sons has been found to be dependent on economic conditions. Indeed, the link between evolutionary and economic concepts may be the single existing bridge in the integrative framework that we envision.

Trying to integrate disparate fields sounds easy in theory, but is hard in practice. Each field has different cultures, conventions, and journals, and one is typically not familiar with the theoretical developments in the other disciplines. But exchange of ideas between anthropology, demography, economics, psychology, sociology, and evolutionary theorizing also promises substantial payoffs: It can guide research and theory more effectively. We agree with Friedman \& Hechter, Hames, Michalski, and Pashos that we need more and better data to test theories of grandparental investment. Ideally, future investigations will be longitudinal, involve myriad process and outcome measures, and encompass several generations. In persuading grant institutions to fund such expensive studies, designing them as interdisciplinary in nature may give them a decisive edge in the scientific marketplace.

\section{R3. What do grandparents do, what should they do, and what are the effects?}

Grandparental investments need not be invariably good. Hoppmann \& Klumb make this point, thus reinforcing the differentiated evidence from traditional societies. Although the maternal grandmother has been suggested as the second most helpful adult family member behind the mother in traditional societies, under some circumstances this help can be detrimental (Sear \& Mace 2008). For example, whereas in the patrilineal Oromo of southern Ethiopia having a maternal grandmother present increased survival of boys to three years of age (Gibson \& Mace 2005), in the matrilineal Chewa of Malawi, the presence of a maternal grandmother reduced the probability of her granddaughter surviving to five years of age (Sear 2008). Likewise, in industrialized nations, the utility that grandparental investments have is highly variable, as numerous commentators emphasized, and, again, it changes across cultures, even within industrialized societies (Rosman \& Yoshikawa 2001). It is patent that not all grandparents invest in their grandchildren or are caring (Fantino \& Stolarz-Fantino); that some grandparents are in competition for the same limited resources as their grandchildren (Fawcett, van den Berg, Weissing, Park, \& Buunk [Fawcett et al.]; Lee), and that when some grandparents help out, it may be seen as interfering or implying parental incompetence (Hoppmann \& Klumb). In order to better understand and predict which investments are beneficial and which are detrimental, respectively, we agree with Michalski that we need to better understand what contemporary grandparents do with and for their grandchildren. 


\section{R3.1. What do grandparents do with and for their grandchildren?}

Grandparents can adopt many beneficial roles from storytellers, family historians, conciliators, and advisors to being sources of encouragement, skill, knowledge, and even wisdom (Michalski and Gurven \& Schniter). Yet, activities that grandparents share with their grandchildren can also represent a mixed blessing. A paradigmatic example is one of the most common behaviors that grandparents and grandchildren share: watching television (Höpflinger et al. 2006). In the best of all worlds, TV consumption can be educational and foster intergenerational dialogue. At the same time, it can foster a sedentary lifestyle. In a study of 84 Native American and African American three-generation families, Polley et al. (2005) found that a grandchild's body mass index (BMI) was positively associated with grandparental BMI. Moreover, a higher grandparental BMI was related to lower activity levels in the grandchild and a lower grandparental activity level was associated with more hours of television watching in the grandchild. Although no attempt was made to establish the independent contribution of these relationships (and they are not surprising, considering the family clustering of BMI across three generations; Guillaume et al. 1995), the study by Polley et al. highlights that researchers must monitor carefully the activities in which grandparents and grandchildren engage. As Michalski points out, summative measures - such as time spent with grandchild may hide the sophisticated things that grandparents can do, and, we would add, also the potentially detrimental activities.

Consequently, both Michalski and Hames demand more detailed information and more detailed measures representing the perspective of both donor and recipient only then can progress be made in understanding the evolved psychological mechanisms by which the donations exert their influence. Relatedly, Euler emphasizes that outcome measures should be broadened to include such measures as grandparental and grandchild happiness. Friedman \& Hechter take the issue of insufficient measures a step further and argue that the current lack of quality longitudinal data thwarts robust empirical tests of the competing predictions from different theories. They appear to believe that sophisticated longitudinal investigations are unlikely in the near future. We are less pessimistic. There are recent longitudinal studies that promise to provide us with richer data, for example, the Millennium Cohort Study (www.cls.ioe.ac.uk/studies.asp?section = 000100020001) or the Longitudinal Study of Australian Children (www.aifs.gov.au/growingup/). Moreover, longitudinal studies are beginning to collect daily activity diaries that detail information on specific behaviors at regular intervals throughout the day (Baxter \& Smith 2009). We are currently on the brink of a data explosion that, if shared across disciplines, will enable the grandparental investment field of research to advance immeasurably.

\section{R3.2. How can we measure the effects?}

Gurven \& Schniter argue that research on grandparental investment implies causality but in reality often demonstrates phenotypic correlations. They call for the explication and investigation of the proximate mechanisms that convert grandparents' behavior into kin welfare consequences, thus rendering it possible to understand the impact of contemporary grandparents' behaviors. We completely agree and also stress that researchers need to be aware of and consider reverse causality. For illustration, consider the following possible causalities: (a) Do grandparents' investments improve grandchildren's cognitive, social, and academic outcomes, or are grandparents more attracted to and responsive to clever, friendlier grandchildren? (b) Do healthier grandparents invest more resources in their grandchildren, or do grandparents who invest more in their grandchildren (within reason) stay healthier? (c) Is living with grandparents bad for grandchildren (Chin \& Xihua 2008; Marks 2006), or do grandparents begin living with grandchildren (e.g., as custodial parents) because of a poor pre-existing environment that impacts grandchild development?

Unfortunately, to make matters more complex, the mechanisms that broker the consequences of grandparental behavior and kin welfare will depend on myriad conditions such as pre-existing family environment. For example, custodial grandparenting is commonly seen as detrimental for both the grandchild's and the grandparent's well-being. How much of this association occurs because of what a grandparent actually does and how much arises because of the pre-existing family environment that precipitated the grandparent taking up this role? In a longitudinal study of American adolescents, Pittman (2007) examined the influence of a grandmother's childcare responsibility and whether she co-resided with her grandchild, and the impact that had on the grandchild's psychological adjustment. Crucially, Pittman adjusted for the grandchild's psychological adjustment at an earlier time point. Co-residing with a grandmother improved psychological adjustment, but it decreased this if the grandparent was custodial. In a more detailed analysis, however, Pittman and Boswell (2007) demonstrated that after moving into a custodial grandparent household grandchildren actually showed improvement and exhibited lower levels of internalizing behaviors (e.g., withdrawal, depression, anxiety) as measured by the Child Behavior Checklist. One evident interpretation is that grandparents become custodial grandparents under the most difficult of conditions, which in themselves are associated with poor childhood outcomes; nevertheless, they still make a difference to their grandchildren's psychological development. From this follows the possibility that custodial grandparents could in reality provide valuable resources that buffer grandchildren against these stressors or moderate their impact. Similarly, Hughes et al. (2007) showed that the negative health effects of being custodial grandparents were largely attributable to grandparents' pre-existing health and socioeconomic characteristics. Clearly, to reveal the authentic effects of grandparental behavior, we need - as Gurven \& Schniter stress - a better understanding of the proximate mechanisms, and in addition, the inclusion of preexisting environment and grandparent and grandchild characteristics in longitudinal study design.

\section{R3.3. What is optimal grandparental investment?}

Coming from different angles, Cox and Fawcett et al. and Gurven \& Schniter raise the question of how grandparents should optimally invest their typically limited resources. Taking an economic perspective, Cox points 
out that grandparental help, like other economic goods or services, is subject to the law of diminishing marginal utility. That is, the better off the grandchild, the smaller the impact of an additional unit of grandparental help. From this follows that utility-maximizing grandparents should distribute their resources such that they get the most bang for their buck. In referring to our review of the beneficial effects of grandparental resources in lowrisk and high-risk conditions, Cox suggests that diminishing marginal utility explains why grandparenting confers less pronounced benefits in the former circumstances, relative to the latter. Advocating a combination of evolutionary and rational actor perspectives into one framework (contrary to Pashos), Gurven \& Schniter explicitly suggest that grandparents should choose to allocate their behavior optimally, such that it can have the highest marginal benefit (at lowest personal costs). As Cox suggests, high-risk contexts could represent circumstances under which the cost-benefit tradeoff of grandparental resources are best, and, consistent with this possibility, Gurven \& Schniter report evidence that in a forager-horticulturalist society that they have studied, grandparents often become primary caretakers when parents die.

We entirely agree with the important role of marginal diminishing utility. But in contrast to Cox's suggestion, we believe it is too early to tell whether the relative lack of evidence for benefits of grandparental investment in low-risk conditions is indeed a consequence of the fact that grandchildren who do well simply derive less marginal utility from additional units of grandparental resources than grandchildren who are worse off. There are just too few published studies on the potential benefits of grandparental resources in low-risk contexts. And their rarity may reflect the reality of researchers and grant institutions "investing" in the study of high-risk conditions because they hope for larger effect sizes and more impact in domains with sociopolitical relevance (e.g., teenage pregnancy). It could well be, however, that in industrialized societies where enormous investments into children are required in order to foster their success, investments under low-risk conditions yield high returns on dimensions such as level of education and future income. The issue, then, is to figure out on which dimensions to look for such high returns.

Like Cox, Fawcett et al. also pick up on the theme of nonlinear returns of grandparental investment, and the question of how optimizing grandparents should distribute their resources. In their analysis, Fawcett et al. assume an evolutionary rather than an economic currency (i.e., "lifetime reproductive success of the grandchild"; see their Figure 1) that is to be optimized. They argue that Trivers' (1974) theory of parent-offspring conflicts over the allocation of parental resources can and should be extended to grandparental resources. Because a child is more related to itself than to its siblings, it seeks a more skewed distribution of resources than parents, being equally related to all their children, are willing to grant. Giving each child some but not all resources also means that parents invest their resources such that the investment yields relatively large marginal utilities. By extension, because a grandchild is more related to itself than to its siblings or cousins, it desires to secure a larger piece of the pie than the grandparents, being equally related to all of their children's children, are willing to give.
Extending Trivers' (1974) framework to grandparental resources is an excellent idea. It will likely yield a set of novel research questions (e.g., how do parents and grandchildren attract and negotiate the allocation of grandparents' resources?), and challenge a perhaps too rosy picture of the grandparent-grandchild relationship (see Friedman \& Hechter). However, a simple one-to-one mapping of Trivers' theory onto grandparental resources and their allocation is difficult, and the picture may be even more complicated than suggested by Fawcett et al. for at least the following reasons: First, the conflict over how to distribute grandparental resources implicates three generations, and parents are likely to act as agents on behalf of their children. Michalski points out that there is not a single study exploring how parents attempt to "encourage" their parents to invest into their grandchildren. Moreover, parents' intermediate roles are complicated by the fact that one of the parents is biologically related to his or her siblings' offspring but the other is not. In other words, parents themselves may operate on the basis of different biases or preferences, depending on the lineage. Finally, the conflict over allocations between parents and offspring occurs in a context of relative transparency and information symmetry; that is, all parties tend to know all relevant information (e.g., a child experiences how a parent treats its siblings). That is less likely to be the case for grandparental resources, with a grandchild being less aware of how much time or resources grandpa and grandma spend on her cousins.

Let us conclude by pointing out that we completely agree with Hames' important point - which carries even more weight in the context of a Triversian analysis of grandparent-parent-grandchild conflicts - that research on grandparental resources needs to better monitor not only what grandparents do with grandchildren, but also what and how much grandparents invest beyond the focal grandchild.

\section{R4. Why do helpers help?}

Several of the commentaries - Broadfield, Kramer, Fantino \& Stolarz-Fantino, Gurven \& Schniter, and Strassmann \& Kurapati - address the question of why grandparents help. One explanation that has attracted wide attention is the grandmother hypothesis, according to which female grandparental investment and reproductive senescence evolved because the intergenerational transfer of affection, help, care, food, and wisdom increases grandmothers' inclusive fitness. Broadfield argues against this ultimate mechanism and suggests that even though grandparental investment is beneficial in modern societies, grandparental altruism is not an adaptation, but an epiphenomenon of an extended lifespan. Specifically, he argues that if menopause was selected for to provide time for grandparental investment, one would expect to see evidence of menopause in our closest animal relatives. But there is none. Moreover, he suggests that life expectancy into post-reproductive years is a recent phenomenon of the twentieth century.

First and foremost, we agree with Broadfield that the existence of benefits of grandparental care does not necessitate grandparental investment to represent an evolved adaptation (see sect. 2.3 of our target article). Nevertheless, we are not as convinced that the 
grandmother hypothesis can be refuted on the grounds outlined in Broadfield's arguments. The evidence that human, chimpanzee, and gorilla follicle depletion rates are similar (Atsalis \& Margulis 2008; Jones et al. 2007) suggests that menopause in humans does have a phylogenetic legacy. Moreover, low life expectancy in historical and traditional societies obviously represents an aggregate measure, and is strongly skewed by those societies' high infant mortality. That is, life expectancy at birth is simply not the same as lifespan. True, life expectancy at birth was less that 50 years in national populations until the twentieth century (Oeppen \& Vaupel 2002), but that does not mean that few lived beyond 50. As Oeppen and Vaupel pointed out, increases in life expectancy until the middle of the twentieth century were due to large reductions in death rates at younger ages. So, what is the mortality signature of human adulthood (i.e., among those who survived childhood)? According to Hawkes (2004; see also, Paine \& Boldsen 2006), first, most girls in hunter-gatherer and historical societies who survived childhood continued to live past their child-bearing years. Second, a third or more of women (of those who are over 15 years of age) usually live beyond the age of 45. Broadfield's arguments are not compelling enough to put the grandmother hypothesis to rest - in particular, when taking into account that Gurven \& Schniter's findings indirectly address Broadfield's argument.

Gurven \& Schniter show (see their Table 1) that two hunter-gatherer societies and two modern societies differ in "age at first grandparenting," namely, 38 years (hunter-gatherers and acculturated hunter-gatherers) versus 58.6 years (Spain) and 50 years (United States). What these numbers imply is that a long life expectancy is not necessary for grandparenting, and that our ancestors - the only great ape simultaneously to raise more than one dependent offspring (see Kramer) - possibly had to decide simultaneously, all before their reproductive years ended, in which of their offspring to invest their scarce resources (Kaplan et al. 2000) and whether to invest in their grandchildren.

How are we to deal with this reproductive overlap and the inescapable competition for resources necessary for reproduction? Overt competition for such resources has been demonstrated in other cooperatively breeding mammals (Clutton-Brock et al. 2006). For humans, Cant and Johnstone (2008) proposed that ceasing reproduction early (menopause) might have been a way to minimize the reproductive overlap between generations, and thus, resource competition. Indeed, despite humans' high generational overlap (see Gurven \& Schniter), they show an extremely low level of reproductive overlap compared to other primates (Cant \& Johnstone). By ceasing reproduction early, grandmothers-to-be would reduce the reproductive conflict between generations, free up resources for younger reproducers, and boost their own inclusive fitness.

Consistent with this hypothesis, overlap between a mother's reproductive period and that of her daughter is uncommon in humans (e.g., Flinn 1989). Should it occur, it has negative consequences for the growth and survival of grandchildren, as demonstrated in a study of nutritional status and mortality in rural Ethiopia. Sear and colleagues (2000) showed that the presence of maternal grandmothers improved their grandchildren's nutritional status (weight and height) up to five years of age. The benefits of a maternal grandmother on height, however, was compromised in children whose maternal grandmothers were still reproducing, with them being significantly shorter relative to those with a post-reproductive maternal grandmother. In fact, their disadvantage was similar to that of children whose maternal grandmother had died. The nutritional benefit translated into improved survival throughout childhood, an effect that was again compromised (but did not reach statistical significance) in children whose grandmothers were still reproducing. In section R7.3, we return to the grandmother hypothesis and to Strassmann \& Kurapati's challenge to its empirical foundation.

Stressing the economic reality of the relationship between generations, Kramer offers an alternative to the grandmother and good-mother hypotheses and suggests economic interdependency across generations as an explanation for grandparental investment. Specifically, she proposes that in traditional societies dietary resources and labor are pooled and transferred from old to young, and vice versa. Moreover, children require fewer resources than previously thought, because they produce some portion of what they need, and even overproduce at some tasks. Consequently, the production conditions of traditional forager and agricultural societies enforce a more equitable and directly reciprocal relationship across generations than is typically assumed, thus reducing the costs of grandparental investment.

By depicting grandparental investment as part of an economic exchange, Kramer builds a bridge to the economic accounts of intergenerational transfers. To consolidate this bridge and to make the notion of a semi-equitable relationship compelling, however, more data on the human economic life cycle are needed. According to Lee, for example, hunter-gatherers are estimated to become net producers of calories as late as age 21 , and continue to be net producers of calories until near their time of death. If grandparental investment were primarily explained in terms of the mutual benefits of reciprocated equitable favors, then would one not expect a more balanced allocation of resources between young and elderly?

Fantino \& Stolarz-Fantino offer still another route toward grandparental altruism, learning, and reinforcement principles. In doing so, they provide a bridge to the economic literature that deserves to be explored. In the economic literature on intergenerational transfers, learning principles have, for example, been invoked in the demonstration-effect hypothesis (Cox \& Stark 2005), according to which, parents who expect to require care and support in old age have an incentive to demonstrate such behavior toward their own parents. The target audience is their children. By observing this model of behavior and the consequences, so the argument goes, the children will be inclined to follow suit and imitate these transfers in the future.

Fantino \& Stolarz-Fantino also suggest that many of us operate on the basis of a generalized social belief that "what goes around, comes around." Such a domaingeneral belief suggests that not every act of altruism, grandparental or otherwise, needs to be directly reciprocated for such behavior to be maintained. Postulating this belief, of course, raises the question of how and why it was learned. Moreover, is it, for instance, a function of 
"payoff to cooperation in everyday life" or "market integration" - two cultural dimensions that have been shown to affect the level of prosociality expressed in experimental games (Henrich et al. 2005)?

\section{R5. Grandparental investment in industrialized societies and reproductive success}

Our starting premise was that in industrialized societies, characterized by a postmodern regime of low fertility and mortality, the effects of grandparental investments will most likely not be observed on classic fitness indicators. Instead, we suspected that they may be found in terms of less tangible dimensions, such as the grandchildren's cognitive and verbal ability, mental health, and wellbeing. Notwithstanding this population-wide regularity, in section 7.3 of our article, we speculated and presented initial data that within subgroups of a population there may be links between grandparental care and fertility. Specifically, parents of working mothers may be key to their daughters' decision to have children, in particular in countries with modest state-financed infrastructure for childcare (e.g., daycare). Several commentators Hoppmann \& Klumb and Kaptijn \& Thomese (but see Pashos) - support this thesis. According to Hoppmann \& Klumb, grandparents can help their children to orchestrate the conflicting demands of production and reproduction, and avoid the frustrating choice between motherhood and career. Kaptijn \& Thomese report having found a link between the availability of grandparental childcare and their children's fertility in a Dutch sample. They also emphasize the role of parents' preferences concerning childcare: Even with state-subsidized infrastructure for childcare in place, prevailing cultural norms may, for example, value private (e.g., grandparental care) over public childcare, thus causing a positive link between grandparental resources and fertility.

Kaptijn \& Thomese also gently remind us that fertility and child mortality are not the be-all-and-end-all of reproductive success, and indicators such as age at first reproduction may, on a population level, still be associated with grandparental investment in industrialized societies. We agree. In fact, we believe that it is helpful to conceptualize grandparental investment not purely as post-reproductive investment, but also as part of the grandparents' lifetime reproductive strategy. A life history perspective suggests that for individuals who develop in a harsh environment, it may be adaptive to reproduce earlier, have more descendants, and invest fewer resources in each descendant (see Sear \& Dickins; Coall \& Chisholm 2010). Consistent with this, we found that grandparents who had their first child and first grandchild at a younger age invested fewer resources in a focal grandchild. Moreover, this association was partially mediated by the higher number of children and grandchildren that these early reproducing grandparents' had (Coall et al. 2009). This initial evidence indicates that examining grandparental investment as the final stage of grandparents' own reproductive strategies could be a fruitful research heuristic.

On a practical level, age of first reproduction is important because, as Sear \& Dickins point out, timing of reproduction is of great concern for policy-makers in the developed world, with the two opposite poles, namely, teenage pregnancy and delaying reproduction to a point when declining fecundity requires the help of the booming fertility industry. On a theoretical level, age of reproduction is challenging because its association with grandparental investment may not be as linear as it has been observed to be in historical and traditional societies (see Kramer). For example, in their classic study of the influence that grandmothers had in two historical populations, Lahdenperä et al. (2004) found that having a grandmother present at the time her offspring (son or daughter) began to reproduce was associated with a 2.4-year earlier age at first reproduction. Grandmothers thus, so the interpretation goes, provide additional resources that improve the parents' condition and increase the probability of earlier reproduction. In contrast, in industrialized societies, as Sear \& Dickins suggest, the influence of grandparental investment could be two-fold: It could delay age of first reproduction if affluent parents and grandparents-to-be were to invest in the quality of their grandchildren by supporting delayed, post-higher-education family planning of their children. Conversely, grandparental investment could bring forward the age of first reproduction if, in contexts of low socioeconomic status, the presence of grandparents were to signal to women that early reproduction is feasible. Currently, there is little direct evidence in support of the latter "kin" hypothesis, which appears to be partially in conflict with the finding that a lack of paternal investment markedly increases the likelihood of teenage pregnancy.

To conclude, whether or not grandparental investment in industrialized societies still impacts fertility and other classic fitness variables such as age of first reproduction is an exciting research question (see Sear \& Dickins). Moreover, unlike Pashos, we see grandparental childcare as a crucial measure of grandparental investment. Dependent on the availability of state-subsidized childcare and cultural norms concerning private and public childcare, there are indications that grandparental care has fitness consequences even in industrialized societies. This association is likely to become more pronounced during difficult times. The current global economic crisis - draining state coffers and imposing financial sacrifices on many with jobs as well as those out of work - may make it more likely that parents turn to family for childcare assistance. How extensive the effect of grandparental childcare is on fertility decisions will depend on the myriad interactions of public policy, availability of institutionalized childcare, rates of women's participation in the workforce, flexible working hours, and fertility. This is exactly the basket of variables that requires - no, demands - a multidisciplinary framework.

\section{R6. Key questions for future research}

Reading the commentaries provided a unique opportunity to recognize some new and exciting research directions. Because of space limitations, we must be selective, and some new research questions that we have already touched upon will not be repeated here (e.g., the relationship of private and public transfers, and the issue of 
crowding in and out; the relationship between life-history indicators and grandparental investment in the modern world).

\section{R6.1. Being a helping grandparent pays}

Our focus and that of most research on grandparental investment has been weighted towards the effects of grandparental investment on grandchildren; however, it is clear that grandparental investment is not a one-way street (see sect. 7.2 in our target article). Several commentators elaborate on the potential benefits that grandparents may gain from their good deeds and the relationship with their children and grandchildren. Euler points out a valuable finding that we missed in Tinsley and Parke (1987), namely, that grandparents reported significantly greater satisfaction with their contact with children and grandchildren than the parents did (p. 270). Taking an evolutionary perspective, Euler suggests that "nepotistic effort" reflects an important "life effort" during the sunset years, and therefore, that such behavior is inherently rewarding. What is striking to us is that Euler's thesis could also have been couched in terms of a lifespan developmental approach, which Hoppmann \& Klumb advocate.

To this end, grandparenting has been recognized as an effective activity that can meet the generative needs of older people (Fisher 1995). In an Australian study of grandparent satisfaction, it was the psychological aspects of grandparenting - including generative concerns, the centrality of the grandparenting role to the individual, and being valued as an elder by their grandchildren - that predicted satisfaction (Thiele \& Whelan 2008). In contrast, the number of hours per week that the grandparent provided childcare was not predictive of satisfaction. Apparently, contact alone is not enough for grandparents to be satisfied with their role; rather, it is the meaning and generativity surrounding this contact that matters. One reason could be that older people, faced with the prospect of a limited future, seek emotionally meaningful experiences (Carstensen et al. 1999), and prioritize generative and emotionally meaningful goals (Lang \& Carstensen 2002).

These and related results suggest that grandparenting - provided that people still enjoy the physical and mental resources necessary - represents the conduit for emotionally gratifying experiences that older people, attempting to regulate their emotions via meaningful activities, seek out. Clearly, the exact psychological and neural mechanisms need to be worked out. However, if even mandatory, tax-like transfers to a charity elicit neural activity in areas linked to reward processing (Harbaugh et al. 2007), we would be surprised if grandparents, voluntarily donating their resources to their kin, did not also receive payback in terms of some neural reward currency.

\section{R6.2. How can one explain young kin's altruism towards elders?}

Cox points out that younger generations' investments into ailing grandparents pose a conceptual difficulty for kin selection theory, and, perhaps because of this, have attracted little attention in the evolutionary literature.
We agree that the evolutionary logic favors downward intergenerational transfers because the reproductive value of younger individuals is generally higher than that of older members in a society (Frank 1998). Consequently, understanding why individuals help older people may not be best achieved from a "gene's-eye view." Economists, for example, have proposed accounts such as the demonstration-effect hypothesis (Cox \& Stark 2005) and the strategic-bequest hypothesis (Bernheim et al. 1985), which predict that resources flow from young (even if the young are selfish) to older generations. Another potentially fruitful direction in which one may find partial answers is the mechanism of empathy (see sect. 4.3 of our target article).

Perhaps one of the greatest achievements of modernization is that societies have built institutions that implement public transfers to the elderly, thus sparing them poverty, neglect, and possibly geronticide (which according to Gurven \& Schniter is commonly practiced in pre-industrial societies). As a result, the economic role of the elderly has been radically changed, with, as Lee points out, a long and healthy old age of extensive consumption and little labor. One topic that repeatedly emerges in public discourse is whether the enormous public resource flow to the older generations is sustainable in light of the large changes in the frequency of old and very old in the developed world (Oeppen \& Vaupel 2002). In this context, one may also ask to what extent the very existence of the substantial private transfers from the old to the young persuades the younger generations to support and accept what Lee identifies as the changed economic role of the elderly.

\section{R6.3. How does what grandparents offer change over time?}

Hames suggests that the grandparental resources being transferred are need-sensitive; that is, initial investments are likely to be in the form of direct grandparental care, such as babysitting. When grandchildren grow older, monetary resources, such as assistance in higher education, may become more pertinent. What is interesting about this plausible hypothesis is that such need- and age-dependent changes in resources may also reflect the capabilities of the aging grandparents. They babysit when they are still relatively young, and transfer monetary resources when their ailing health makes it increasingly impossible to be directly involved. Age of grandparents and grandchildren, of course, is a variable associated with a number of changes worth studying. For example, contact, but not necessarily closeness, in the grandparent-grandchild relationship decreases as children grow up (Dench \& Ogg 2002; but see also, Bridges et al. 2007). With both generations getting older, their views of each other change (Kahana \& Kahana 1970), which, in turn, is likely to affect how grandchildren try to elicit investment, and what type of investment grandparents offer.

\section{R6.4. Beyond classic fitness indicators in investigations of traditional societies}

In order to find the effects of grandparental investments in industrialized societies, we recommended focusing on dimensions such as the grandchildren's cognitive and verbal ability, mental health, and well-being. This is in 
contrast to evolutionary psychologists' and anthropologists' focus on classic fitness indicators in traditional societies. Gurven \& Schniter rightly point out that dimensions beyond fitness indicators (e.g., transfer of knowledge) also deserve to be studied in traditional societies, even more so because such societies epitomize high-risk contexts, in which the most important grandparental resource - in particular, later in the child's life - may not be calories but, for example, skills.

\section{R7. Things we got wrong, or did we?}

Our target article was admittedly ambitious, trying to bring together evidence and explanations of grandparental investment stemming from different disciplines. In doing so, we entered territory new to us, and therefore omissions and errors may have been inevitable. In previous sections, we have addressed oversights as the opportunity arose. Now we turn to three broader challenges to some generalizations we proposed.

\section{R7.1. Do grandparents have more or less opportunity to care in industrialized societies?}

Gurven \& Schniter argue that grandparental opportunity was higher in hunter-gatherer societies than in modern industrialized societies, contrary to our conclusion. We appreciate their analysis of demographic parameters across different societies. At the same time, however, we also caution against overrating the parameters' value, as they fail to recognize several important properties of grandparents and of grandparents environments in industrial societies that are likely to increase the opportunity to invest. First, today's grandparents are likely to be healthier than their predecessors and invest over a longer period. Second, they have more resources (in particular, through the enormous institutional upward transfers that they receive; see Lee). Third, they have substantially fewer grandchildren in whom to invest (see the total fertility rate in Gurven \& Schniter's Table 1), and therefore, there is less competition for grandparental resources within generations. Fourth, the wider-spaced generations are likely to reduce competition for grandparental resources between generations (see Fawcett et al.). Based on these differences between grandparenting in the past and today, we believe that our conclusion - that the opportunity for grandparents to care is higher than ever - still holds.

\section{R7.2. How traditional are modern societies?}

Huber takes us to task for arguing that in industrialized societies many of the links that held in ancestral times links between grandparental help and inclusive fitness, links between economic status and reproductive success, and sex-biased grandparental investment (TriversWillard theory) - still hold. The links are more hidden, but they are still there. We respect Huber's conviction in the robustness of evolutionary regularities in modern societies, and agree that trying to uncover them, should they still exist, is a worthy research enterprise.

\section{R7.3. Are grandparents helpful or not?}

Strassmann \& Kurapati provide a thorough analysis, which they suggest shows - in contrast to a previous review (Sear \& Mace 2008) on which we relied heavily that grandparents in traditional societies are not, or not strongly, beneficial when measured in terms of child survival. Therefore, so they conclude, referring to humans as cooperative breeders is misplaced, or at least overemphasized. Specifically, Strassmann \& Kurapati expand Sear and Mace's review by detailing and quantifying all associations between each of the four grandparent types being present (or alive) and grandchild survival, which is split further into effects on grandsons and granddaughters. With the space limitations, Strassmann \& Kurapati obviously had little opportunity to detail their procedure, and therefore, it is difficult for us to evaluate their analysis in detail. However, let us share some of our observations.

According to Strassmann \& Kurapati, most of the associations between the maternal grandmother, the grandparent whose impact should be largest, and child survival are non-significant. This is indeed true in $73 \%$ of analyses. However, the remaining associations were overwhelmingly positive, rather than negative $(22 \%$ vs. $0.5 \%)$. This is unlikely to be a chance finding. We also noticed that the negative effect of the maternal grandmother found by Beise (2005), with a relative risk of 0.79 , was taken to be statistically significant at $p<.1$ for girls, whereas the positive effect of the maternal grandmother found by Gibson and Mace (2005), with an odds ratio of 0.714 (and, on inspection of Table 1 in their original article [p. 474], a 95\% confidence interval that does not cross $1[0.51-0.99])$, was taken to be non-significant for boys. For others to replicate Strassmann \& Kurapati's analysis, it would be important to know the standards that the authors applied (which could, for example, be made accessible in a document on their website).

A final observation that we made is that Strassmann \& Kurapati's detailed analysis should encourage researchers to specify the time course of the predicted effects more precisely, and thus to test them more stringently. Take, for illustration, the Beise (2005) data set. It shows that the maternal grandmother has no effect from 0-11 months of age, which is when most infants are being breastfed; it has a positive effect in three out of four analyses between 12 and 36 months of age, when provisioning of children moves to alloparents, and becomes negative only for girls between 36 and 59 months of age. Obviously, our summary is post-hoc, but it does suggest that the initial lack of effects and the subsequent positive effects could be consistent with the role of grandparents as alloparents, thus supporting the proposition that humans are cooperative breeders. Indeed, we suspect that not even the greatest believer in the merits of grandparental investment would predict that benefits hold invariably across all ages and ecologies. Finally, on a technical note, counting the number of negative, positive, or non-significant associations could be highly misleading, when the coarseness of the bins changes across time. For example, the period from $0-11$ months, in which one may predict no effect, is divided into three bins in Beise (2005; see Table 1 in Strassmann \& Kurapati), whereas the period from 12 to 23 months, for which one may predict an effect, is collapsed into one bin only.

Notwithstanding these issues, we applaud Strassmann \& Kurapati's efforts, even if Sear and Mace's analysis is not as rudimentary as this commentary suggests (see the "other effects and notes" column in Table 2A and 2B; 
Sear \& Mace 2008). We hope that their contribution will ensure a constructive debate on the relative merits of the grandmother and mother hypotheses.

\section{R8. Conclusion}

We are convinced that to move forward, research on grandparental investment and intergenerational transfers, scattered across disciplines, will need to embrace an ethos of interdisciplinary research. Our target article aimed to take first steps toward a multidisciplinary framework. Generally, the commentaries we received echo this need to travel across disciplinary borders. After reading the thoughtful questions and issues brought up by our commentators we are more than ever persuaded that a joint effort by researchers from psychology, sociology, anthropology, evolutionary biology, and demography will reveal so much more about the effects of grandparenting in the developed world than can a single discipline on its own.

\section{References}

[Letters “ $a$ " and " $r$ " appearing before authors' initials refer to target article and response references, respectively.]

Albertini, M., Kohli, M. \& Vogel, C. (2007) Intergenerational transfers of time and money in European families: Common patterns - different regimes? Journal of European Social Policy 17:319-34. [aDAC]

Aldous, J. (1995) New views of grandparents in intergenerational context. Journal of Family Issues 16:104-22. [aDAC]

Alexander, R. D. (1974) The evolution of social behavior. Annual Review of Ecology and Systematics 5:325-83. [aDAC]

Alexander, R. D. (1987) The biology of moral systems. Aldine de Gruyter. [HAE]

Alger, I. \& Weibull, J. (forthcoming) Kinship, incentives and evolution. American Economic Review. [DC]

American Academy of Pediatrics (1997) Breastfeeding and the use of human milk. Pediatrics 100:1035-39. [aDAC]

Anderson, K. G. (2005) Relatedness and investment in children in South Africa. Human Nature 16:1-31. [aDAC]

Anderson, K. G. (2006) How well does paternity confidence match actual paternity? Evidence from worldwide nonpaternity rates. Current Anthropology 47:513-20. [aDAC, MV]

Anderson, K. G., Kaplan, H. \& Lancaster, J. B. (2007) Confidence of paternity, divorce, and investment in children by Albuquerque men. Evolution and Human Behavior 28:1-10. [aDAC]

Andreoni, J. (1990) Impure altruism and donations to public goods: A theory of warm-glow giving. Economic Journal 100:464-77. [aDAC]

Apfel, N. H. \& Seitz, V. (1991) Four models of adolescent mother-grandmother relationships in Black inner-city families. Family Relations 40:421-29. [aDAC]

Apostolou, M. (2007) Sexual selection under parental choice: The role of parents in the evolution of human mating. Evolution and Human Behavior 28:403-09. [TWF]

Arias, E. (2006) United States life tables, 2003. National vital statistics reports (Vol. 54, No. 14). National Center for Health Statistics. [aDAC]

Arrondel, L. \& Masson, A. (2006) Altruism, exchange or indirect reciprocity: What do the data on family transfers show? In: Handbook of the economics of giving, altruism and reciprocity, vol. 2: Applications, ed. S. C. Kolm \& J. M. Ythier, pp. 971-1053. Handbooks in Economics 23. North-Holland/ Elsevier. [aDAC]

Atsalis, S. \& Margulis, S. W. (2008) Perimenopause and menopause: Documenting life changes in aging female gorillas. Interdisciplinary Topics in Gerontology 36:119-46. [DCB, arDAC]

Atsalis, S. \& Videan, E. N. (2009) Functional versus operational menopause: Reply to Herndon \& Lacreuse. American Journal of Primatology 71:893-94. [DCB]

Attar-Schwartz, S., Tan, J.-P., Buchanan, A., Flouri, E. \& Griggs, J. (2009) Grandparenting and adolescent adjustment in two-parent biological, loneparent, and step-families. Journal of Family Psychology 23:67-75. [aDAC]

Attias-Donfut, C., Ogg, J. \& Wolff, F. C. (2005) European patterns of intergenerational financial and time transfers. European Journal of Ageing $2: 161-73$. [aDAC]
Attias-Donfut, C. \& Segalen, M. (2002) The construction of grandparenthood. Current Sociology 50:281-94. [aDAC]

Aubel, J. (2005) Grandmothers: A learning institution. Retrieved December 7 , 2007, from: http://www.grandmotherproject.org/Art.GM.JIC_files/GM.TomeFinal.pdf [aDAC]

Aubel, J., Touré, I. \& Diagne, M. (2004) Senegalese grandmothers promote improved maternal and child nutrition practices: The guardians of tradition are not averse to change. Social Science and Medicine 59:945-59. [aDAC]

Austad, S. N. (1994) Menopuase: An evolutionary perspective. Experimental Gerontology 29:255-63. [aDAC]

Baltes, P. B. (1997) On the incomplete architecture of human ontogeny: Selection, optimization, and compensation as foundation of developmental theory. American Psychologist 52:366-80. [rDAC]

Baltes, P. B., Smith, J. \& Staudinger, U. M. (1992) Wisdom and successful aging. In: Nebraska Symposium on Motivation, vol. 39, ed. T. B. Sonderegger, pp. 12367. University of Nebraska Press. [MG]

Banks, J. W. (2003) Ka’nisténhsera Teiakotíhsnie’s. A native community rekindles the tradition of breastfeeding. AWHONN Lifelines 7:340-47. [aDAC]

Barrett, L., Dunbar, R. \& Lycett, J. (2002) Human evolutionary psychology. Palgrave. [aDAC]

Barro, R. J. (1974) Are government bonds net wealth? Journal of Political Economy 82:1095-17. [aDAC]

Barry, D. (1984) Babies and other hazards of sex, p. 54. Rodale [aDAC]

Bateman, A. J. (1948) Intra-sexual selection in Drosophila. Heredity 2:349-68. [aDAC]

Bauer, T. \& Strub, S. (2002) Ohne Krippe Grosi stünde Vieles still [Without grandparental daycare, many things would come to a standstill]. Input for "Forum Familienfragen" der Eidgenössischen Koordinationskommission für Familenfragen, Solothurn, Switzerland. Büro für arbeits- und sozialpolitische Studie, Berne, \& Swiss National Science Foundation. [aDAC]

Baxter, J. \& Smith, J. (2009) Breastfeeding and infants' time use (Research Paper No. 43). Australian Institute of Family Studies, Melbourne. [rDAC]

Beck, C. T. (2001) Predictors of postpartum depression. An update. Nursing Research 50:275-85. [aDAC]

Becker, G. S. (1974) A theory of social interactions. Journal of Political Economy 82:1063-93. [aDAC]

Becker, G. S. (1991) A treatise on the family. Harvard University Press. [aDAC]

Becker, G. S. \& Tomes, N. (1976) Child endowments and the quantity and quality of children. Journal of Political Economy 84:S143-S162. [aDAC]

Becker, G. S. \& Tomes, N. (1979) An equilibrium theory of the distribution of income and intergenerational mobility. Journal of Political Economy 87:115389. [aDAC $]$

Beise, J. (2005) The helping grandmother and the helpful grandmother: The role of maternal and paternal grandmothers in child mortality in the 17th and 18th century population of French settlers in Quebec, Canada. In: Grandmotherhood: The evolutionary significance of the second half of female life, ed. E. Voland, A. Chasiotis \& W. Schiefenhövel, pp. 215-38. Rutgers University Press. [rDAC, BIS]

Bellis, M. A., Hughes, K. E., Hughes, S. K. \& Ashton, J. R. (2005) Measuring paternal discrepancy and its public health consequences. Journal of Epidemiology and Community Health 59:749-54. [MV]

Bengtson, V. L. (2001) Beyond the nuclear family: The increasing importance of multigenerational bonds. Journal of Marriage and Family 63:1-16. [arDAC]

Bengtson, V. L. \& Roberts, R. E. L. (1991) Intergenerational solidarity in aging families: An example of formal theory construction. Journal of Marriage and Family 53:856-70. [rDAC]

Bentley, M. E., Caulfield, L. E., Gross, S. M., Bronner, Y., Jensen, J., Kessler, L. A \& Paige, D. M. (1999) Sources of influence on intention to breastfeed among African-American women at entry to WIC. Journal of Human Lactation 15:27-34. [aDAC]

Bentley, M. E., Dee, D. L. \& Jensen, J. L. (2003) Breastfeeding among low income, African-American women: Power, beliefs and decision making. Journal of Nutrition 133:305-09. [aDAC]

Bergstrom, T. C. (1997) Economics in a family way. Journal of Economic Literature 34:1903-34. [DC]

Bernheim, B. D. \& Severinov, S. (2000) Bequests as signals: An explanation for the equal division puzzle (NBER Working Paper No. 7791). National Bureau of Economic Research. [aDAC]

Bernheim, B. D., Shleifer, A. \& Summers, L. H. (1985) The strategic bequest motive. Journal of Political Economy 93:1045-76. [rDAC]

Beutler, L. E. (1991) Have all won and must all have prizes? Revisiting Luborsky et al.'s verdict. Journal of Consulting and Clinical Psychology 59:226-32. $[\mathrm{MV}]$

Billari, F.C. \& Kohler, H.-P. (2004) Patterns of low and lowest-low fertility in Europe. Population Studies 58:161-76. [aDAC]

Billari, F. C., Kohler, H.-P., Andersson, G. \& Lundström, H. (2007) Approaching the limit: Long-term trends in late and very late fertility. Population and Development Review 33:149-70. [aDAC] 
Bishop, D. I., Meyer, B. C., Schmidt, T. M. \& Gray, B. R. (2009) Differential investment behavior between grandparents and grandchildren: The role of paternity uncertainty. Evolutionary Psychology 7:66-77. [arDAC]

Black, M. M. \& Nitz, K. (1996) Grandmother co-residence, parenting, and child development among low income, urban teen mothers. Journal of Adolescent Health 18:218-26. [aDAC]

Black, M. M., Papas, M. A., Hussey, J. M., Hunter, W., Dubowitz, H., Kotch, J. B., English, D. \& Schneider, M. (2002) Behavior and development of preschool children born to adolescent mothers: Risk and 3-generation households. Pediatrics 109:573-80. [aDAC]

Black, M. M., Siegel, E. H., Abel, Y. \& Bentley, M. E. (2001) Home and videotape intervention delays early complementary feeding among adolescent mothers. Pediatrics 107(5):e67. [aDAC]

Blake, J. (1987) Differential parental investment: Its effects on child quality and status attainment. In: Parenting across the life span: Biosocial dimensions, ed. J. B. Lancaster, J. Altmann, A. S. Rossi \& L. R. Sherrod, pp. 351-75. Aldine de Gruyter. [aDAC]

Bliege Bird, R. \& Bird, D. (2002) Constraints of knowing or constraints of growing? Fishing and collecting by the children of Mer. Human Nature 13:239-67. [KLK]

Blurton Jones, N., Hawkes, K. \& O’Connell, J. (1989) Measuring and modeling costs of children in two foraging societies: Implications for schedule of reproduction. In: Comparative socioecology. The behavorial ecology of humans and other mammals, ed. V. Standen \& R. Foley, pp. 367-90. Blackwell Scientific. [KLK]

Blurton Jones, N., Hawkes, K. \& O'Connell, J. (1997) Why do Hadza children forage? In: Uniting psychology and biology: Integrative perspectives on human development, ed. N. Segal, G. E. Weisfeld \& C. C. Weisfeld, pp. 164-83. American Psychological Association. [KLK]

Blurton Jones, N., Hawkes, K. \& O’Connell, J. (2002) Antiquity of postreproductive life: Are there modern impacts on hunter-gatherer postreproductive life spans? American Journal of Human Biology 14:184-205. [aDAC]

Bogin, B. (1997) Evolutionary hypotheses for human childhood. Yearbook of Physical Anthropology 40:63-89. [aDAC]

Bogin, B. \& Smith, B. H. (1996) Evolution of the human life cycle. American Journal of Human Biology 8:703-16. [DCB, aDAC]

Borgerhoff Mulder, M. (1998) The demographic transition: Are we any closer to an evolutionary explanation? Trends in Ecology and Evolution 13:266-70. [arDAC]

Borgerhoff Mulder, M. (2007) Hamilton's rule and kin competition: The Kipsigis case. Evolution and Human Behavior 28:299-312. [aDAC, BIS]

Borgerhoff Mulder, M., Bowles, S., Hertz, T., Bell, A., Beise, J., Clark, G., Fazzio, I., Gurven, M., Hill, K., Hooper, P. L. Irons, W., Kaplan, H., Leonetti, D., Low, B., Marlowe, F., McElreath, R., Naidu, S., Nolin, D., Piraino, P., Quinlan, R., Schniter, E., Sear, R., Shenk, M., Smith, E., von Rueden, C. \& Wiessner, P. (2009) Intergenerational wealth transmission and the dynamics of inequality in small-scale societies. Science 326(5953):682-88. [MG]

Börsch-Supan, A. \& Miegel, M., eds. (2001) Pension reform in six countries: What can we learn from each other? Springer. [aDAC]

Botcheva, L. B. \& Feldman, S. S. (2004) Grandparents as family stabilizers during economic hardship in Bulgaria. International Journal of Psychology 39:15768. [aDAC]

Brandon, P. D., Heyworth, C. \& Griffen, L. (2007) The rise of three-generation households among two-parent and single-parent families. The Household, Income, and Labour Dynamics in Australia (HILDA) Survey. Retrieved November 20, 2007, from: http://melbourneinstitute.com/hilda/conf/conf2007/ HILDA\%20Conf\%20Papers\%202007/All\%20Papers/Brandon,\%20Peter_final\%20paper.pdf [aDAC]

Brandt, M., Haberkern, K. \& Szydlik, M. (2009) Intergenerational help and care in Europe. European Sociological Review 25:585-601. [rDAC]

Bridges, L. J., Roe, A. E. C., Dunn, J. \& O'Connor, T. G. (2007) Children’s perspectives on their relationships with grandparents following parental separation: A longitudinal study. Social Development 16:539-54. [arDAC]

Brosnan, S. F. \& de Waal, F. B. M. (2003) Monkeys reject unequal pay. Nature 425:297-99. [aDAC]

Brosnan, S. F., Schiff, H. C. \& de Waal, F. B. M. (2005) Tolerance for inequity may increase with social closeness in chimpanzees. Proceedings of the Royal Society of London B: Biological Sciences 272:253-58. [aDAC]

Brown, S. L., Nesse, R. M., Vinokur, A. D. \& Smith, D. M. (2003) Providing social support may be more beneficial than receiving it: Results from a prospective study of mortality. Psychological Science 14:320-27. [aDAC]

Brown, W. M., Consedine, N. S. \& Magai, C. (2005) Altruism relates to health in an ethnically diverse sample of older adults. The Journals of Gerontology. Series B: Psychological Sciences and Social Sciences 60B:P143-52. [aDAC]
[aD.

Burkart, J. M., Fehr, E., Efferson, C. \& van Schaik, C. P. (2007) Other-regarding preferences in a non-human primate: Common marmosets provision food altruistically. Proceedings of the National Academy of Sciences USA 104:19762-66. [aDAC]
References/Coall \& Hertwig: Grandparental investment

Burton, L. M. (1990) Teenage childbearing as an alternative life-course strategy in multigeneration black families. Human Nature 1:123-43. [aDAC]

Buunk, A. P., Park, J. H. \& Dubbs, S. L. (2008) Parent-offspring conflict in mate preferences. Review of General Psychology 12:47-62. [TWF]

Buunk, A. P., Park, J. H. \& Duncan, L. A. (in press) Cultural variation in parental influence on mate choice. Cross-Cultural Research. (DOI: 10.1177/ 1069397109337711) [TWF]

Burton, L. M. (1992) Black grandparents rearing children of drug-addicted parents: Stressors, outcomes, and social service needs. The Gerontologist 32:744-51. [aDAC]

Cain, M. (1977) The economic activities of children in a village in Bangladesh. Population and Development Review 3:201-27. [KLK]

Caldwell, J. C. (1976) Toward a restatement of demographic transition theory. Population and Development Review 2:321-66. [RH]

Caldwell, J. C., Caldwell, B. K., Caldwell, P., McDonald, P. F. \& Schindlmayr, T. (2006) Demographic Transition Theory. Springer. [aDAC]

Campbell, C. \& Lee, J. Z. (1996) A death in the family: Household structure and mortality in rural Liaoning: Life-event and time-series analysis, 1792-1867. The History of the Family 1(3):297-328. [BIS]

Cant, M. A. \& Johnstone, R. A. (2008) Reproductive conflict and the separation of reproductive generations in humans. Proceedings of the National Academy of Sciences USA 105:5332-36. [arDAC]

$\mathrm{Cao}, \mathrm{H}$. (2006) Time and financial transfers within and beyond the family. Journal of Family and Economic Issues 27:375-400. [aDAC]

Carstensen, L. L. \& Lockenhoff, C. E. (2003) Aging, emotion and evolution: The bigger picture. Annals of the New York Academy of Sciences 1000:152-79. [MG]

Caputo, R. K. (1999) Age-condensed and age-gapped families: Coresidency with elderly parents and relatives in a mature women's cohort, 1967-1995. Marriage and Family Review 29:77-95. [aDAC]

Carey, J. R. \& Judge, D. S. (2001) Life span extension in humans is self-reinforcing: A general theory of longevity. Population and Development Review 27:41136. [aDAC]

Caro, T. M., Sellen, D. W., Parish, A., Frank, R., Brown, D. M., Voland, E. \& Borgerhoff Mulder, M. (1995) Termination of reproduction in nonhuman and human female primates. International Journal of Primatology 16:205-20. [aDAC]

Carstensen, L. L., Isaacowitz, D. M. \& Charles, S. T. (1999) Taking time seriously: A theory of socioemotional selectivity. American Psychologist 54:165-81. [rDAC]

Case, A., Lin, I.-F. \& McLanahan, S. (2001) Educational attainment of siblings in stepfamilies. Evolution and Human Behavior 22:269-89. [HAE]

Case, A. \& Paxson, C. (2001) Mothers and others: Who invests in children's health? Journal of Health Economics 20:301-28. [HAE]

Caspari, R. \& Lee, S.-H. (2004) Older age becomes common late in human evolution. Proceedings of the National Academy of Sciences USA 101:10895900. [DCB, aDAC]

Cater, S. \& Coleman, L. (2006) "Planned" teenage pregnancy: Perspectives of young parents from disadvantaged backgrounds. Joseph Rowntree Foundation. The Policy Press. [RS]

Central Intelligence Agency (2009) The World Factbook. Retrieved August 20, 2009 from: https://www.cia.gov/library/publications/the-world-factbook/rankorder/ 2127rank.html. [aDAC]

Chan, C. G. \& Elder, G. H., Jr. (2000) Matrilineal advantage in grandchildgrandparent relations. The Gerontologist 40:179-90. [rDAC]

Chase-Lansdale, P. L., Brooks-Gunn, J. \& Zamsky E. S. (1994) Young AfricanAmerican multigenerational families in poverty: Quality of mothering and grandmothering. Child Development 65:373-93. [aDAC]

Chin, M. M. \& Xihua, Z. (2008) Family and motivation effects on mathematics achievement: Analyses of students in 41 countries. Learning and Instruction 18:321-36. [rDAC]

Chrastil, E. R., Getz, W. M., Euler, H. A. \& Starks, P. T. (2006) Paternity uncertainty overrides sex chromosome selection for preferential grandparenting. Evolution and Human Behavior 27:206-23. [aDAC]

Christensen, K., Doblhammer, G., Rau, R. \& Vaupel, J. W. (2009) Ageing populations: The challenges ahead. Lancet 374:1196-1208. [DCB]

Cigno, A. (1993) Intergenerational transfers without altruism: Family, market and state. European Journal of Political Economy 9:505-18. [aDAC]

Cigno, A. (2006) The political economy of intergenerational cooperation. In: Handbook of the economics of giving, altruism and reciprocity, vol. 2: Applications, ed. S. C. Kolm \& J. M. Ythier, pp. 1505-58. Handbooks in Economics 23. North-Holland/Elsevier. [aDAC]

Clarke, A. L. \& Low, B. S. (2001) Testing evolutionary hypotheses with demographic data. Population and Developmental Review 27:633-60. [rDAC, BRH]

Cloutier, C., Broadfield, D. C., Wolf, T. \& Halloran, A. R. (2009) A semi-free ranging chimpanzee (Pan troglodytes) gives birth at 65 years of age: Implications for delayed reproductive senescence. American Journal of Physical Anthropology (Suppl.) 48:107. [DCB] 
Clutton-Brock, T. H. (1989) Mammalian mating systems. Proceedings of the Royal Society of London B: Biological Sciences 236:339-72. [aDAC]

Clutton-Brock, T. H. (1991) The evolution of parental care. Princeton University Press. [aDAC]

Clutton-Brock, T. H. (2002) Breeding together: Kin selection and mutualism in cooperative vertebrates. Science 296:69-72. [KLK]

Clutton-Brock, T. H., Hodge, S. J., Spong, G., Russell, A. F., Jordan, N. R., Bennett, N.C., Sharpe, L. L. \& Manser, M. B. (2006) Intrasexual competition and sexual selection in cooperative mammals. Nature 444:1065-68. [rDAC]

Coall, D. A. \& Chisholm, J. S. (2010) Reproductive development and parental investment during pregnancy: Moderating influence of mother's early environment. American Journal of Human Biology 22:143-53. [rDAC]

Coall, D. A. \& Hertwig, R. (submitted) Grandparental investment: A relic of the past or a resource for the future? Current Directions in Psychological Science. [rDAC]

Coall, D. A., Meier, M., Hertwig, R., Wänke, M. \& Höpflinger, F. (2009) Grandparental investment: The influence of reproductive timing and family size. American Journal of Human Biology 21:455-63. [arDAC]

Cockburn, A. (2006) Prevalence of different modes of parental care in birds. Proceedings of the Royal Society of London B 273:1375-83. [BIS]

Cohen, A. A. (2004) Female post-reproductive lifespan: A general mammalian trait. Biological Reviews 79:733-50. [aDAC]

Cohen, J. (1965) Some statistical issues in psychological research. In: Handbook of clinical psychology, ed. B. B. Wolman, pp. 95-121. McGraw-Hill. [aDAC]

Cohen, J. (1988) Statistical power analysis for the behavioral sciences, 2nd edition. Erlbaum. [aDAC]

Cohen, S. \& Wills, T. A. (1985) Stress, social support, and the buffering hypothesis Psychological Bulletin 98:310-57. [aDAC, CAH]

Cooney, T. M. \& An, J. S. (2006) Women in the middle: Generational position and grandmothers' adjustment to raising grandchildren. Journal of Women and Aging 18(2):3-24. [aDAC]

Council of Europe (2005) Recent demographic developments in Europe, 2004. Council of Europe Publishing. [aDAC]

Cox, C. B. (2000) Empowering grandparents raising grandchildren: A training manual for group leaders. Springer. [aDAC]

Cox, D. \& Fafchamps, M. (2008) Extended family and kinship networks: Economic insights and evolutionary directions. In: Handbook of development economics, vol. 4, ed. T. P. Schultz \& J. Strauss, pp. 3711-84. Elsevier. [DC]

Cox, D. \& Stark, O. (2005) On the demand for grandchildren: Tied transfers and the demonstration effect. Journal of Public Economics 89:1666-97. [rDAC, DC]

Cremer, H. \& Pestieau, P. (2006) Wealth transfer taxation: A survey of the theoretical literature. In: Handbook of the economics of giving, altruism and reciprocity, vol. 2: Applications, ed. S. C. Kolm \& J. M. Ythier, pp. 1107-34. Handbooks in Economics 23. North-Holland/Elsevier. [aDAC]

Crookston, B. T., Dearden, K. A., Chan, K., Chan, T. \& Stoker, D. D. (2007) Buddhist nuns on the move: An innovative approach to improving breastfeeding practices in Cambodia. Maternal and Child Nutrition 3:10-24. [aDAC]

Crosnoe, R. \& Elder G. H. (2002) Life course transitions, the generational stake, and grandparent-grandchild relationships. Journal of Marriage and Family 64:1089-96. [aDAC]

Daly, M. \& Wilson, M. (1980) Discriminative parental solicitude: A biological perspective. Journal of Marriage and Family 42:277-88. [arDAC]

Dawkins, R. (2006) The selfish gene, 30th anniversary edition. Oxford University Press. [aDAC]

Dench, G. \& Ogg, J. (2002) Grandparenting in Britain: A baseline study. Institute of Community Studies, London. [rDAC]

Denham, T. E. \& Smith, C. W. (1989) The influence of grandparents on grandchildren: A review of the literature and resources. Family Relations 38:34550. [aDAC, MG]

Dennis, C. L. (2005) Psychosocial and psychological interventions for prevention of postnatal depression: Systematic review. British Medical Journal 331:15-23. [aDAC]

de Quervain, D. J., Fischbacher, U., Treyer, V., Schellhammer, M., Schnyder, U., Buck, A. \& Fehr, E. (2004) The neural basis of altruistic punishment. Science 305:1254-58. [aDAC]

Derosas, R. (2002) Fatherless families in 19th-century Venice. In: When Dad died: Individuals and families coping with family stress in past societies, ed. R. Derosas \& M. Oris, pp. 421-52. Peter Lang. [BIS]

d'Espaignet, T. D., Measey, M. L., Carnegie, M. A. \& Mackerras, D. (2003) Monitoring the "Strong women, strong babies, strong culture program": The first eight years. Journal of Paediatrics and Child Health 39:668-872. [aDAC]

de Waal, F. (2006) Primates and philosophers. How morality evolved. Princeton University Press. [aDAC]

de Waal, F. B. M. (2008) Putting the altruism back into altruism: The evolution of empathy. Annual Review of Psychology 59:279-300. [aDAC, KLK] de Waal, F. B. M., Leimgruber, K. \& Greenberg, A. R. (2008) Giving is selfrewarding for monkeys. Proceedings of the National Academy of Sciences USA 105:13685-89. [aDAC]

Drew, L. M. \& Silverstein, M. (2007) Grandparents' psychological well-being after loss of contact with their grandchildren. Journal of Family Psychology 21:37279. [aDAC]

Drew, L. M. \& Smith, P. K. (2002) Implications for grandparents when they lose contact with their grandchildren: Divorce, family feud, and geographical separation. Journal of Mental Health and Aging 8:95-119. [aDAC]

Dubas, J. S. (2001) How gender moderates the grandparent-grandchild relationship: A comparison of kin-keeper and kin-selector theories. Journal of Family Issues 22:478-92. [arDAC]

Duflo, E. C. (2003) Grandmothers and granddaughters: Old age pensions and intrahousehold allocation in South Africa. The World Bank Economic Review 17:1-25. [HAE]

Duong, D. V., Lee, A. H. \& Binns, W. (2005) Determinants of breast-feeding within the first 6 months post-partum in rural Vietnam. Journal of Paediatrics and Child Health 41:338-43. [aDAC]

Duschek, K.-J. \& Wirth, H. (2005) Kinderlosigkeit von Frauen im Spiegel des Mikrozensus. Eine Kohortenanalyse der Mikrozensen 1987 bis 2003 [Childlessness of women as reflected in the micro census. A cohort analysis from 1987 to 2003]. Wirtschaft und Statistik 8:800-20. Retrieved from: http://www.unituebingen.de/frauenvertreterin/download/Kinderlosigkeit.pdf [CAH]

Dyson, T. \& Murphy, M. (1985) The onset of fertility transition. Population and Development Review 11:399-440. [KLK]

Economist, The (2009) More or less equal? A special report on the rich [Electronic version of The Economist, April 2, 2009]. Retrieved from: http://www.economist.com/specialreports/displaystory.cfm?story_id $=13356650 \quad[\mathrm{aDAC}]$

Edwards, O. W. (2006) Teachers' perceptions of the emotional and behavioral functioning of children raised by grandparents. Psychology in the Schools 43:565-72. [aDAC]

Eggebeen, D. J. (1992) Family structure and intergenerational exchanges. Research on Aging 14:427-47. [aDAC]

Eisenberg, A. R. (1988) Grandchildren's perspectives on relationships with grandparents: The influence of gender across generations. Sex Roles 19:20517. [aDAC]

Ekström, A., Widström, A. M. \& Nissen, E. (2003) Breastfeeding support from partners and grandmothers: Perceptions of Swedish women. Birth 30:26166. [aDAC $]$

Ellison, P. T. (1990) Human ovarian function and reproductive ecology: New hypotheses. American Anthropologist 92:933-52. [aDAC]

Ellison, P. T. (2001) On fertile ground: A natural history of human reproduction. Harvard University Press. [DCB, aDAC]

Emery Thompson, M., Jones, J. H., Pusey, A. E., Brewer-Marsden, S., Goodall, J., Marsden, D., Matsuzawa, T., Nishida, T., Reynolds, V., Sugiyama, Y. \& Wrangham, R. W. (2007) Aging and fertility patterns in wild chimpanzees provide insights into the evolution of menopause. Current Biology 17:2150-56. [DCB]

Erikson, E. H. (1966) Eight ages of man. International Journal of Psychiatry 2:281300. [rDAC, $\mathrm{CAH}]$

Esbensen, B. A., Østerlind, K. \& Hallberg, I. R. (2007) Quality of life of elderly persons with cancer: A 6-month follow-up. Scandinavian Journal of Caring Sciences 21:178-90. [aDAC]

Esping-Andersen, G. (1990) The three worlds of welfare capitalism. Polity Press. [RK]

Essock-Vitale, S. M. \& McGuire, M. T. (1985) Women's lives viewed from an evolutionary perspectives. II. Patterns of helping. Ethology and Sociobiology 6:155-73. [aDAC]

Euler, H. A., Hoier, S. \& Rohde, P. A. (2001) Relationship-specific closeness of intergenerational family ties. Findings from evolutionary psychology and implications for models of cultural transmission. Journal of Cross-Cultural Psychology 32:147-58. [aDAC, RLM]

Euler, H. A. \& Michalski, R. L. (2007) Grandparental and extended kin relationships. In: Family relationships: An evolutionary perspective, ed. C. Salmon \& T. K. Shackelford, pp. 185-204. Oxford University Press. [aDAC, RLM]

Euler, H. A. \& Weitzel, B. (1996) Discriminative grandparental solicitude as reproductive strategy. Human Nature 7:39-59. [arDAC, MV]

Falbo, T. (1991) The impact of grandparents on children's outcomes in China Marriage and Family Review 16:369-76. [aDAC]

Fantino, E. \& Kennelly, A. (2009) Sharing the wealth: Factors influencing resource allocation in the sharing game. Journal of the Experimental Analysis of Behavior 91(3):337-54. [EF]

Fantino, E. \& Stolarz-Fantino, S. (2002) The role of negative reinforcement; or: Is there an altruist in the house? Behavioral and Brain Sciences 25:257-58. [EF]

Fedigan, L. M. \& Pavelka, M. S. M. (2001) Is there adaptive value to reproductive termination in Japanese macaques? A test of maternal investment hypotheses. International Journal of Primatology 22:109-25. [aDAC]

Fehr, E. \& Gächter, S. (2002) Altruistic punishment in humans. Nature 415:13740. [aDAC, EF] 
Fehr, E. \& Schmidt, K. M. (2006) The economics of fairness, reciprocity and altruism: Experimental evidence and new theories. In: Handbook of the economics of giving, altruism and reciprocity, vol. 1: Foundations, ed. S. C. Kolm \& J. M. Ythier, pp. 615-91. Handbooks in Economics 23. NorthHolland/Elsevier. [aDAC]

Fergusson, E., Maughan, B. \& Golding, J. (2008) Which children receive grandparental care and what effect does it have? Journal of Child Psychology and Psychiatry 49:161-69. [aDAC]

Finch, J. \& Mason, J. (1993) Negotiating family responsibilities. Routledge. [aDAC]

Fisher, B. J. (1995) Successful aging, life satisfaction, and generativity in late life. International Journal of Aging and Human Development 41:239-50. [rDAC]

Fisher, J. R. W., Hammarberg, K. \& Baker, H. W. G. (2005) Assisted conception is a risk factor for postnatal mood disturbances and early parenting difficulties. Fertility and Sterility 84:426-30. [aDAC]

Fisher, R. A. (1930) The genetical theory of natural selection. Clarendon Press. [aDAC]

Flinn, M. V. (1988) Parent-offspring interactions in a Caribbean village: Daughter guarding. In: Human reproductive behavior: A Darwinian perspective, ed. L. Betzig, M. Borgerhoff Mulder \& P. Turke, pp. 189-200. Cambridge University Press. [KLK]

Flinn, M. V. (1989) Household composition and female reproductive strategies. In: Sexual and reproductive strategies, ed. A. Rasa, C. Vogel \& E. Voland, pp. 206-33. Chapman and Hall. [rDAC]

Frank, S. A. (1998) Foundations of social evolution. Princeton University Press. [rDAC]

Friedman, D., Hechter, M. \& Kanazawa, S. (1994) A theory of the value of children. Demography 31(3):375-401. [DF]

Friedman, D., Hechter, M. \& Kreager, D. (2008) A theory of the value of grandchildren. Rationality and Society 20(1):31-63. [arDAC, DF, TWF]

Frone, M. (2000) Work-family conflict and employee psychiatric disorders: The national comorbidity survey. Journal of Applied Psychology 85:888-95. [CAH

Fujiwara, T. \& Lee, C. K. (2008) The impact of altruistic behaviors for children and grandchildren on major depression among parents and grandparents in the United States: A prospective study. Journal of Affective Disorders 107:2936. [aDAC]

Fuller-Thomson, E. (2005) Canadian First Nations grandparents raising grandchildren: A portrait in resilience. International Journal of Aging and Human Development 60:331-42. [aDAC]

Fuller-Thomson, E. \& Minkler, M. (2000) African American grandparents raising grandchildren: A national profile of demographic and health characteristics. Health and Social Work 25:109-18. [aDAC]

Galinsky, E., Salmond, K., Bond, J. T., Kropf, M. B., Moore, M. \& Harrington, B. (2003) Leaders in a global economy. A study of executive women and men. Center for Work and Family in Boston College. [CAH]

Gaulin, S. J. C. (1980) Sexual dimorphism in the human post-reproductive life-span: Possible causes. Journal of Human Evolution 9:227-32. [aDAC]

Gaulin, S. J. C., McBurney, D. H. \& Brakeman-Wartell, S. L. (1997) Matrilateral biases in the investment of aunts and uncles: A consequence and measure of paternity uncertainty. Human Nature 8:139-51. [MV]

Gaulin, S. J. C. \& Schlegel, A. (1980) Paternal confidence and paternal investment A cross cultural test of a sociobiological hypothesis. Ethology and Sociobiology $1: 301-309$. [aDAC $]$

Geary, D. C. (2000) Evolution and proximate expression of human parental investment. Psychological Bulletin 126:55-77. [aDAC]

Gibson, M. A. \& Mace, R. (2005) Helpful grandmothers in rural Ethiopia: A study of the effect of kin on child survival and growth. Evolution and Human Behavior 26:469-82. [arDAC, BIS]

Gibson, M. A. \& Mace, R. (2006) An energy-saving development initiative increases birth rate and childhood malnutrition in rural Ethiopia. PLoS Medicine 3:e87. [aDAC]

Goh, E. C. L. (2009) Grandparents as childcare providers: An in-depth analysis of the case of Xiamen, China. Journal of Aging Studies 23:60-68. [aDAC]

Goodman, A. \& Koupil, I., (2009) Social and biological determinants of reproductive success in Swedish males and females born 1915-1929. Evolution and Human Behavior 30:329-41. [BRH]

Gordon, R. A., Chase-Lansdale, P. L. \& Brooks-Gunn, J. (2004) Extended households and the life course of young mothers: Understanding the associations using a sample of mothers with premature, low birth weight babies. Child Development 75:1013-38. [aDAC]

Griffiths, P., Hinde, A. \& Matthews, Z. (2001) Infant and child mortality in three culturally contrasting states of India. Journal of Biosocial Science 33(4):603-22. [BIS]

Grundy, E. \& Henretta, J. C. (2006) Between elderly parents and adult children: A new look at the intergenerational care provided by the "sandwich generation". Ageing and Society 26:707-22. [aDAC]

Guillaume, M., Lapidus, L., Beckers, F., Lambert, A. \& Bjorntorp, P. (1995) Family trends of obesity through three generations: The Belgian-Luxembourg child
References/Coall \& Hertwig: Grandparental investment

study. International Journal of Obesity and Related Metabolic Disorders 19(Suppl. 3):5-9. [rDAC]

Gurven, M. \& Kaplan, H. (2007) Longevity among hunter-gatherers: A crosscultural examination. Population and Development Review 33:321-65. [aDAC, MG]

Gurven, M. \& Kaplan, H. (2008) Beyond the Grandmother Hypothesis: Evolutionary models of longevity. In: Cultural context of aging: Worldwide perspectives, 3rd edition, ed. J. Sokolovsky, pp. 53-60. Praeger. [MG]

Güth, W., Schmittberger, R. \& Schwarze, B. (1982) An experimental analysis of ultimatum bargaining. Journal of Economic Behavior and Organization 3:36788. [aDAC]

Guzman, L. (1999) The use of grandparents as child care providers. National Survey of Families and Households Working Paper No. 84. Retrieved July 15, 2008, from: http://www.ssc.wisc.edu/cde/nsfhwp/nsfh84.pdf [aDAC $]$

Guzman, L. (2004) Grandma and grandpa taking care of the kids: Patterns of involvement. Child Trends Research Brief. Retrieved July 15, 2008, from: http://www.childtrends.org/Files/GrandparentsRB.pdf [aDAC]

Hadley, C. (2004) The costs and benefits of kin: Kin networks and children's health among the Pimbwe of Tanzania. Human Nature 15:377-95. [aDAC]

Hagen, E. H. (1999) The functions of postpartum depression. Evolution and Human Behavior 20:325-59. [aDAC]

Hagen, E. H., Barrett, H. C. \& Price, M. E. (2006) Do human parents face a quantity-quality tradeoff?: Evidence from a Shuar community. American Journal of Physical Anthropology 130:405-18. [aDAC]

Hakim, C. (2002) Lifestyle preferences as determinants of women's differentiated labor market careers. Work and Occupations 29:428-59. [RK]

Hakim, C. (2003) A new approach to explaining fertility patterns: Preference theory. Population and Development Review 29:349-74. [RK]

Hames, R. B. (1987) Relatedness and garden labor exchange among the Ye'kwana. Evolution and Human Behavior 8:354-92. [RH]

Hames, R. B. (1988) The allocation of parental care among the Ye'kwana. In: Human reproductive behavior: A Darwinian perspective, ed. L. Betzig, M. Borgerhoff Mulder, \& P. Turke, pp. 237-51. Cambridge University Press. [aDAC]

Hames, R. B. \& Draper, P. (2004) Women's work, child care and helpers at the nest in a hunter-gatherer society. Human Nature 15:319-41. [RH]

Hamilton, W. D. (1964) The genetical evolution of social behaviour (I and II) Journal of Theoretical Biology 7:1-52. [arDAC]

Hamilton, W. D. (1966) The moulding of senescence by natural selection. Journal of Theoretical Biology 12:12-45. [aDAC]

Hammarberg, K. \& Clarke, V. E. (2005) Reasons for delaying childbearing: A survey of women aged over 35 years seeking assisted reproductive technology. Australian Family Physician 34:187-89. [aDAC]

Hammer, M. L. A. \& Foley, R. A. (1996) Longevity and life history in hominid evolution. Human Evolution 11:61-66. [aDAC]

Hank, K. \& Buber, I. (2009) Grandparents caring for their grandchildren: Findings from the 2004 Survey of Health, Ageing and Retirement in Europe. Journal of Family Issues 30:53-73. [aDAC]

Hank, K. \& Kreyenfeld, M. (2003) A multilevel analysis of child care and women's fertility decisions in western Germany. Journal of Marriage and Family 65:584-96. [CAH]

Harbaugh, W. T., Mayr, U. \& Burghart, D. R. (2007) Neural responses to taxation and voluntary giving reveal motives for charitable donations. Science 316:1622-25. [rDAC]

Hartung, J. (1985) Matrilineal inheritance: New theory and analysis. Behavioral and Brain Sciences 8:661-88. [aDAC]

Havighurst, R. J. (1948/1972) Developmental tasks and education, 3rd edition. McKay. (Original work published 1948). [rDAC]

Havighurst, R. J. (1953) Human development and education. Longmans, Green. [CAH]

Hawkes, K. (2004) Human longevity: The grandmother effect. Nature 428:12829. $[\mathrm{rDAC}]$

Hawkes, K., O’Connell, J. F. \& Blurton Jones, N. G. (1989) Hardworking Hadza grandmothers. In: Comparative socioecology: The behavioural ecology of humans and other mammals, ed. V. Standen \& R. A. Foley, pp. 341-66. Basil Blackwell. [aDAC, RH, KLK]

Hawkes, K., O’Connell, J. F. \& Blurton Jones, N. G. (1997) Hadza women's time allocation, offspring provisioning and the evolution of long postmenopausal life spans. Current Anthropology 38:551-77. [KLK]

Hawkes, K., O’Connell, J. F., Blurton Jones, N. G., Alvarez, H. \& Charnov, E. L. (1998) Grandmothering, menopause and the evolution of human life histories. Proceedings of the National Academy of Sciences USA 95:1336-39. [arDAC]

Hawkes, K. \& Paine, R. R., eds. (2006) The evolution of human life histories. School of American Research. [KLK]

Hayslip, B. \& Kaminski, P. L. (2005) Grandparents raising their grandchildren: A review of the literature and suggestions for practice. The Gerontologist 45:262-69. [aDAC] 
Healy, M. C. (2000) Perceived acceptance from grandparents and grandchild adjustment. Doctoral dissertation, Department of Psychology, Temple University, Philadelphia. Dissertation Abstracts International, DAI-B 61/10, p. 5565. (AAT 9990320) [aDAC]

Henrich, J., Boyd, R., Bowles, S., Camerer, C., Fehr, E., Gintis, H., McElreath, R., Alvard, M., Barr, A., Ensminger, J., Smith Henrich, N., Hill, K., Gil-White, F., Gurven, M., Marlowe, F.W., Patton, J.Q. \& Tracer, D. (2005) "Economic man" in cross-cultural perspective: Ethnography and experiments from 15 smallscale societies. Behavioral and Brain Sciences 28:795-815. [rDAC]

Herring, D. J. (2008) Kinship foster care: Implications of behavioral biology research. Buffalo Law Review 56:495-556. [HAE]

Hersher, L., Richmond, J. \& Moore, A. (1963) Modifiability of the critical period for the development of maternal behavior in sheep and goats. Behaviour 20:311-20. [EF]

Hertwig, R., Davis, J. N. \& Sulloway, F. J. (2002) Parental investment: How an equity motive can produce inequality. Psychological Bulletin 128:728-45. [aDAC]

Hess, C. R., Papas, M. A. \& Black, M. M. (2002) Resilience among African American adolescent mothers: Predictors of positive parenting in early infancy. Journal of Pediatric Psychology 27:619-29. [aDAC]

Hewlett, B. S. (1991) Demography and childcare in preindustrial societies. Journal of Anthropological Research 47:1-37. [MG]

Heywood, E. M. (1999) Custodial grandparents and their grandchildren. The Family Journal 7:367-72. [aDAC]

Hill, K. (1993) Life history theory and evolutionary anthropology. Evolutionary Anthropology 2:78-88. [aDAC]

Hill, K., Boesch, C., Goodall, J., Pusey, A., Williams, J. \& Wrangham, R. (2001) Mortality rates among wild chimpanzees. Journal of Human Evolution 40:43750. [DCB]

Hill, K. \& Hurtado, A. M. (1991) The evolution of premature reproductive senescence and menopause in human females: An evaluation of the "grandmother hypothesis." Human Nature 2:313-50. [aDAC]

Hill, K. \& Hurtado, A. M. (1996) Ache life history: The ecology and demography of a foraging people. Aldine de Gruyter. [aDAC, BIS]

Hill, K. \& Kaplan, H. S. (1999) Life history traits in humans: Theory and empirical studies. Annual Review of Anthropology 28:397-430. [BRH]

Hill, T. J. (2006) Grandchild, grandparent, and parent coresidence from 1970 to 1990: Structural factors affecting state patterns. International Journal of Aging and Human Development 62:117-42. [aDAC]

Hoddinott, P., Tappin, D. \& Wright, C. (2008) Breast feeding. Clinical Review. British Medical Journal 336:881-87. [aDAC]

Hoem, J. M. (2008) The impact of public policies on European fertility. Demographic Research 19:249-59. [RK]

Hoff, A. (2007) Patterns of intergenerational support in grandparent-grandchild and parent-child relationships in Germany. Aging and Society 27:643-65. [aDAC]

Hofferth, S. L. \& Anderson, K. G. (2003) Are all dads equal? Biology versus marriage as a basis for paternal investment. Journal of Marriage and Family 65:21332. [aDAC]

Hoffman, E. (1980) Young adults' relations with their grandparents: An exploratory study. International Journal of Aging and Human Development 10:299-310. [aDAC]

Hogan, D. P., Eggebeen, D. J. \& Clogg, C. C. (1993) The structure of intergenerational exchanges in American families. American Journal of Sociology 98:1428-58. [aDAC]

Hoorens, S., Gallo, F., Cave, J. A. K. \& Grant, J. C. (2007) Can assisted reproductive technologies help to offset population ageing? An assessment of the demographic and economic impact of ART in Denmark and UK. Human Reproduction 22:2471-75. [aDAC]

Hopcroft, R. L. (2005) Parental status and differential investment in sons and daughters: Trivers-Willard revisited. Social Forces 83(3):1111-36. [BRH]

Hopcroft, R. L. (2006) Sex, status, and reproductive success in the contemporary United States. Evolution and Human Behavior 27:104-20. [BRH]

Höpflinger, F., Hummel, C. \& Hugentobler, V. (2006) Teenage grandchildren and their grandparents in urban Switzerland. Retrieved March 31, 2006 from: http://www.nfp52.ch/e_dieprojekte.cfm?Projects.Command $=$ detailsandget $=13$. $\quad[\operatorname{arDAC}]$

Hoppmann, C. \& Klumb, P. (2004) Grandparental childcare provision: Does it contribute to the developmental success of both grandparents and adult offspring? Paper presented at the 57th Annual Meeting of the Gerontological Society of America, Washington, D.C., November 2004. [CAH]

Hoppmann, C. \& Klumb, P. (2006) Daily goal pursuits predict cortisol secretion and mood states in employed parents with preschool children. Psychosomatic Medicine 68:887-94. [CAH]

Howell, N. (in press) Life histories of the Dobe !Kung. University of California Press. [RDL]

Hrdy, S. B. (1981) The woman that never evolved. Harvard University Press. [aDAC]
Hrdy, S. B. (1999) Mother nature: Maternal instincts and how they shape the human species. Pantheon Books. [aDAC]

Hrdy, S. B. (2005a) Comes the Child before Man: How cooperative breeding and prolonged postweaning dependence shaped human potentials. In: Huntergatherer childhoods: Evolutionary, developmental and colonial perspective, ed. B. S. Hewlett \& M. E. Lamb, pp. 65-91. Aldine Transaction. [aDAC, RH] Hrdy, S. B. (2005b) Cooperative breeders with an ace in the hole. In: Grandmotherhood: The evolutionary significance of the second half of female life, ed. E. Voland, A. Chasiotis \& W. Schiefenhövel, pp. 295-317. Rutgers University Press. [aDAC, BIS]

Hrdy, S. B. (2009) Mothers and others: The evolutionary origins of mutual understanding. Harvard University Press. [aDAC, EF]

Huber, B. R. \& Breedlove, W. L. (2007) Evolutionary theory, kinship, and childbirth in cross-cultural perspective. Cross-Cultural Research 41:196-219. [aDAC]

Huber, B. R., Linhartova, V., Cope, D. \& Lacy, M. (2004) Evolutionary theory and birth-related investment by kin in cross-cultural perspective. World Cultures 15:60-79. [aDAC]

Hughes, M. E., Waite, L. J., LaPierre, T. A. \& Luo, Y. (2007) All in the family: The impact of caring for grandchildren on grandparents' health. The Journals of Gerontology. Series B: Psychological Sciences and Social Sciences 62B:S108S119. [arDAC]

Hurme, H. (2006) Grandparents as resource factors in the family. In: Socioemotional development and health from adolescence to adulthood, ed. L. Pulkkinen, J. Kaprio \& R. J. Rose. Cambridge University Press. [aDAC]

Hyun, O. K., Lee, W., Yoo, A. J., Cho, B. H., Miller, B. C., Schvaneveldt, J. D. \& Lau, S. (2002) Social support for two generations of new mothers in selected populations in Korea, Hong Kong, and the United States. Journal of Comparative Family Studies 33:515-27. [aDAC]

Ingram, J., Johnson, D. \& Greenwood, R. (2002) Breastfeeding in Bristol: Teaching good positioning, and support from fathers and families. Midwifery 18:87101. [aDAC]

Ingram, J., Johnson, D. \& Hamid, N. (2003) South Asian grandmothers' influence on breast feeding in Bristol. Midwifery 19:318-27. [aDAC]

Ivey, P. K. (2000) Cooperative reproduction in Ituri forest hunter-gatherers: Who cares for Efe infants? Current Anthropology 41:856-66. [aDAC]

Izar, P., Verderane, M., Visalberghi, E., Ottoni, E., De Oliveira, M., Shirley, J. \& Fragaszy, D. (2006) Cross-genus adoption of a marmoset (Callithrix jacchus) by wild capuchin monkeys (Cebus libidinosus): Case report. American Journal of Primatology 68:692-700. [EF]

Jamison, C. S., Cornell, L. L., Jamison, P. L. \& Nakazato, H. (2002) Are all grandmothers equal? A review and a preliminary test of the "grandmother hypothesis" in Tokugawa Japan. American Journal of Physical Anthropology 119:67-76. [aDAC, BIS]

Jensen, K., Hare, B., Call, J. \& Tomasello, M. (2006) What's in it for me? Self-regard precludes altruism and spite in chimpanzees. Proceedings of the Royal Society of London B: Biological Sciences 273:1013-21. [aDAC]

Johns, S. E., Dickins, T. E. \& Clegg, H. (in submission) Teenage pregnancy and motherhood: How might evolutionary theory inform policy? [RS]

Johnson, J., Canning, J., Kaneko, T., Pru, J. K. \& Tilly, J. L. (2004) Germline stem cells and follicular renewal in the postnatal mammalian ovary. Nature 428:145-50. [aDAC]

Jones, K. P., Walker, L. C., Anderson, D., Lacreuse, A., Robson, S. L. \& Hawkes, K. (2007) Depletion of ovarian follicles with age in chimpanzees: Similarities to humans. Biology of Reproduction 77:247-51. [DCB, arDAC]

Joslin, D. \& Harrison, R. (2002) Self-reported physical health among older surrogate parents to children orphaned and affected by HIV disease. AIDs Care 14:619-24. [aDAC]

Judge, D. S. \& Carey, J. R. (2000) Postreproductive life predicted by primate patterns. The Journals of Gerontology. Series A: Biological Sciences and Medical Sciences 55A:B201-B209. [aDAC]

Kahana, B. \& Kahana, E. (1970) Grandparenthood from the perspective of the developing grandchild. Developmental Psychology 3:98-105. [arDAC]

Kalil, A., Spencer, M. S., Spieker, S. J. \& Gilchrist, L. D. (1998) Effects of grandmother coresidence and quality of family relationships on depressive symptoms in adolescent mothers. Family Relations 47:433-41. [aDAC]

Kalra, S. K. \& Molinaro, T. A. (2008) The association of in vitro fertilization and perinatal morbidity. Seminars in Reproductive Medicine 26:423-35. [aDAC]

Kaplan, H. (1994) Evolutionary and wealth flows theories of fertility: Empirical tests and new models. Population and Development Review 20(1):753-91. [aDAC, RH, KLK, RDL]

Kaplan, H. (1996) A theory of fertility and parental investment in traditional and modern human societies. Yearbook of Physical Anthropology 39:91-135. [aDAC]

Kaplan, H. (1997) The evolution of the human life course. In: Between Zeus and the salmon: The biodemography of longevity, ed. K. W. Wachter \& C. E. Finch, pp. 175-211. National Academy Press. [aDAC] 
Kaplan, H., Hill, K., Lancaster, J. \& Hurtado, A. M. (2000) A theory of human life history evolution: Diet, intelligence, and longevity. Evolutionary Anthropology 9:156-85. [arDAC]

Kaptijn, R., Thomese, F., van Tilburg, T. G., Liefbroer, A. C. \& Deeg, D. J. H. (2010) Low fertility in contemporary humans and the mate value of their children: Sex-specific effects on social status indicators. Evolution and Human Behavior 31:59-68. [rDAC]

Kellam, S. G., Ensminger, M. E. \& Turner, R. J. (1977) Family structure and the mental health of children: Concurrent and longitudinal community-wide studies. Archives of General Psychiatry 34:1012-22. [aDAC]

Kemkes-Grottenthaler, A. (2005) Of grandmothers, grandfathers and wicked stepgrandparents: Differential impact of paternal grandparents on grandoffspring survival. Historical Social Research 30(3):219. [BIS]

Kennelly, A. \& Fantino, E. (2007) The sharing game: Fairness in resource allocation as a function of incentive, gender, and recipient types. Judgment and Decision Making 2:204-16. [EF]

Kenny, M. E., Blustein, D. L., Chaves, A., Grossman, J. M. \& Gallagher, L. A. (2003) The role of perceived barriers and relational support in the educational and vocational lives of urban high school students. Journal of Counseling Psychology 50:142-55. [aDAC]

King, V., Silverstein, M., Elder, G. H., Jr., Bengtson, V. L. \& Conger, R. D. (2003) Relations with grandparents: Rural Midwest versus urban Southern California. Journal of Family Issues 24:1044-69. [AP]

Kirkwood, T. B. L. (1977) Evolution of ageing. Nature 270:301-04. [aDAC]

Kivett, V. R. (1993) Racial comparisons of the grandmother role: Implications for strengthening the family support system of older black women. Family Relations 42:165-72. [aDAC]

Klumb, P. L. \& Gemmiti, M. (in press) Harmonisierung von Arbeits- und Privatleben [Harmonizing work and private life]. In: Betriebliche Gesundheitsförderung und Gesundheitsmanagement, ed. E. Bamberg, A. Ducki \& A.-M. Metz. Hogrefe. [CAH]

Kohler, H.-P., Billari, F. C. \& Ortega, J. A. (2002) The emergence of lowest-low fertility in Europe during the 1990s. Population and Development Review 28:641-80. [aDAC]

Kohli, M. (1999) Private and public transfers between generations: Linking the family and the state. European Societies 1:81-104. [arDAC, RK]

Kohli, M. (2004) Intergenerational transfers and inheritance: A comparative view. In: Intergenerational relations across time and place (Annual Review of Gerontology and Geriatrics, vol. 24), ed. M. Silverstein, pp. 266-89. Springer [aDAC]

Kohli, M. \& Künemund, H. (2003) Intergenerational transfers in the family: What motivates giving? In: Global aging and challenges to families, ed. V. L. Bengtson \& Lowenstein, pp. 123-42. Aldine de Gruyter. [aDAC]

Kohli, M., Künemund, H., Motel A. \& Szydlik M. (2000) Grunddaten zur Lebenssituation der 40-85 jährigen deutschen Bevölkerung. Ergebnisse des Alters-Survey [Basic data on the living situation of 40- to 85-year-old Germans: Result of the age survey]. Weißensee. [aDAC]

Komdeur, J. (1992) Importance of habitat saturation and territory quality for evolution of cooperative breeding in the Seychelle's warbler. Nature 358:49395. [BIS]

Korchmaros, J. D. \& Kenny, D. A. (2001) Emotional closeness as a mediator of the effect of genetic relatedness on altruism. Psychological Science 12:262-65. [AP]

Korkeila, K., Korkeila, J., Vahtera, J., Kivimaki, M., Kivela, S.-L., Sillanmaki, L. \& Koskenvuo, M. (2005) Childhood adversities, adult risk factors and depressiveness: A population study. Social Psychiatry and Psychiatric Epidemiology 40:700-06. [aDAC]

Kotlikoff, L. J. \& Summers, L. H. (1981) The role of intergenerational transfers in aggregate capital accumulation. Journal of Political Economy 89:706-32 [aDAC]

Kramer, K. L. (2002) Variation in juvenile dependence: Helping behavior among Maya children. Human Nature 13:299-325. [aDAC]

Kramer, K. L. (2004) Reconsidering the cost of childbearing: The timing of children's helping behavior across the life cycle of Maya families. In: Socioeconomic aspects of human behavioral ecology, ed. M. Alvard, pp. 335-53. Elsevier. [KLK]

Kramer, K. L. (2005a) Children's help and the pace of reproduction: Cooperative breeding in humans. Evolutionary Anthropology 14:224-37. [aDAC, KLK, BIS]

Kramer, K. L. (2005b) Maya children: Helpers at the farm. Harvard University Press. [KLK

Kramer, K. L. \& Greaves, R. D. (2007) Changing patterns of infant mortality and fertility among Pumé foragers and horticulturalists. American Anthropologist 109:713-26. [KLK]

Kramer, K. L. \& McMillan, G. P. (2006) The effect of labor saving technology on longitudinal fertility changes. Current Anthropology 47:165-72. [KLK]
[K

Kuhle, B. X. (2007) An evolutionary perspective on the origin and ontogeny of menopause. Maturitas 57:329-37. [DCB]
References/Coall \& Hertwig: Grandparental investment

Künemund, H. \& Rein, M. (1999) There is more to receiving than needing: Theoretical arguments and empirical explorations of crowding in and crowding out. Ageing and Society 19:93-121. [rDAC]

Kuo, Z. Y. (1930) The genesis of the cat's responses to the rat. Journal of Comparative Psychology 11:1-36. [EF]

Kurland, J. A. (1979) Paternity, mother's brother, and human sociality. In: Evolutionary biology and human social behavior: An anthropological perspective, ed. N. A. Chagnon \& W. G. Irons, pp. 145-80. Duxbury Press. [aDAC]

Lachs, M. S. \& Pillemer, K. (2004) Elder abuse. Lancet 364:1263-72. [MG]

Ladusing, L. \& Singh, C. H. (2006) Place, community education, gender and child mortality in north-east India. International Journal of Population Geography 12(1):65-76. [BIS]

Laferrère, A. \& Wolff, F.-C. (2006) Microeconomic models of family transfers. In: Handbook of the economics of giving, altruism and reciprocity, vol. 2: Applications, ed. S. C. Kolm \& J. M. Ythier, pp. 889-969. Handbooks in Economics 23. North-Holland/Elsevier. [aDAC, TWF]

Laham, S. M., Gonsalkorale, K. \& von Hippel, W. (2005) Darwinian grandparenting: Preferential investment in more certain kin. Personality and Social Psychology Bulletin 31:63-72. [aDAC]

Lahdenperä, M., Lummaa, V., Helle, S., Tremblay, M. \& Russell, A. F. (2004) Fitness benefits of prolonged post-reproductive lifespan in women. Nature 428:178-81. [arDAC]

Lahdenperä, M., Russell, A. F. \& Lummaa, V. (2007) Selection for long lifespan in men: Benefits of grandfathering? Proceedings of the Royal Society of London B: Biological Sciences 274:2437-44. [aDAC]

Lancaster, J. B. (1971) Play-mothering: The relations between juvenile females and young infants among free-ranging vervet monkeys (Cercopithecus aethiops). Folia Primatologica 15:161-82. [AP]

Lancaster, J. B., Kaplan, H. S., Hill, K. \& Hurtado, A. M. (2000) The evolution of life history, intelligence, and diet among chimpanzees and human foragers. In: Perspectives in ethology: Evolution, culture, and behavior, vol. 13, pp. 47-72. Plenum. [aDAC]

Lancaster, J. B. \& King, B. J. (1985) An evolutionary perspective on menopause. In: In her prime: A new view of middle-aged women, ed. V. Kerns \& J. K. Brown, pp. 13-20. Bergen and Garvey. [aDAC]

Lancaster, J. B. \& Lancaster, C. S. (1983) Parental investment: The hominid adaptation. In: How humans adapt: A biocultural odyssey, ed. D. Ortner, pp. 33-66. Proceedings of the Smithsonian's Seventh International Symposium. Smithsonian Institution. [aDAC]

Lang, F. R. \& Carstensen, L. L. (2002) Time counts: Future time perspective, goals, and social relationships. Psychology and Aging 17:125-39. [rDAC]

Lavers, C. A. \& Sonuga-Barke, E. J. S. (1997) Annotation: On the grandmothers' role in the adjustment and maladjustment of grandchildren. Journal of Child Psychology and Psychiatry 38:747-53. [aDAC]

Lawson, D. W. \& Mace, R. (2008) Sibling configuration and childhood growth in contemporary British families. International Journal of Epidemiology 37:1408-21. [BIS]

Lawson, D. W. \& Mace, R. (2009) Trade-offs in modern parenting: A longitudinal study of sibling competition for parental care. Evolution and Human Behavior 30:170-83. [aDAC]

Lee, E., Clements, S., Ingham, R. \& Stone, N. (2004) A matter of choice? Explaining national variation in teenage abortion and motherhood. The Joseph Rowntree Foundation. York Publishing Services. [RS]

Lee, R. D. (1997) Intergenerational relations and the elderly. In: Between Zeus and the salmon: The biodemography of longevity, ed. K. W. Wachter \& C. E. Finch pp. 212-33. National Academy Press. [aDAC]

Lee, R. D. (2003a) Rethinking the evolutionary theory of aging: Transfers, not births, shape senescence in social species. Proceedings of the National Academy of Sciences of the United States of America 100:9637-42. [aDAC]

Lee, R. D. (2003b) The demographic transition: Three centuries of fundamental change. Journal of Economic Perspectives 17:167-90. [aDAC]

Lee, R. D. (2008) Sociality, selection, and survival: Simulated evolution of mortality with intergenerational transfers and food sharing. Proceedings of the National Academy of Sciences USA 105:7124-28. [aDAC]

Lee, R. D., Ensminger, M. E. \& LaVeist, T. A. (2005) The responsibility continuum: Never primary, coresident and caregiver - Heterogeneity in the AfricanAmerican grandmother experience. International Journal of Aging and Human Development 60:295-304. [aDAC]

Lee, R. D. \& Kramer, K. L. (2002) Children's economic roles in the Maya family life cycle: Cain, Caldwell and Chayanov revisited. Population and Development Review 28:475-99. [KLK]

Lee, R. D., Lee S-H. \& Mason, A. (2006) Charting the economic life cycle. National Bureau of Economic Research, Working Paper No. 12379. [rDAC]

Lee, R. D., Lee S-H. \& Mason, A. (2008) Charting the economic life cycle. In: Population aging, human capital accumulation, and productivity growth, ed. A. Prskawetz, D. E. Bloom \& W. Lutz. (A supplement to Population and Development Review, vol. 33). Population Council of New York. [RDL] 
Lee, S., Colditz, G., Berkman, L. \& Kawachi, I. (2003) Caregiving to children and grandchildren and risk of coronary heart disease in women. American Journal of Public Health 93:1939-44. [aDAC]

Leek, M. \& Smith, P. K. (1991) Cooperation and conflict in three-generation families. In: The psychology of grandparenthood, ed. P. K. Smith, pp. 177-94. Routledge. [aDAC]

Leonetti, D. L., Nath, D. C. \& Hemam, N. S. (2007) In-law conflict. Women’s reproductive lives and the roles of their mothers and husbands among the matrilineal Khasi. Current Anthropology 48:861-90. [aDAC]

Leonetti, D. L., Nath, D. C., Hemam, N. S. \& Neill, D. B. (2005) Kinship organization and the impact of grandmothers on reproductive success among the matrilineal Khasi and patrilineal Bengali of northeast India. In: Grandmotherhood: The evolutionary significance of the second half of female life, ed. E. Voland, A. Chasiotis \& W. Schiefenhövel, pp. 194-214. Rutgers University Press. [aDAC, BIS]

Levitt, M. J., Weber, R. A. \& Clark, M. C. (1986) Social network relationships as sources of maternal support and well-being. Developmental Psychology 22:310-16. [aDAC]

Lin, C.-H. (2003) Intergenerational parallelism of self-efficacy: Moderating variables, mediating variables, and common antecedents. Doctoral dissertation, Department of Sociology, Texas A\&M University, Dissertation Abstracts International, DAI-A 65/07, p. 2783. (AAT 3141190). [aDAC]

Livi-Bacci, M. (2001) Too few children and too much family. Daedalus 130:13955. [rDAC]

Low, B. S. (2000) Why sex matters. Princeton University Press. [BRH]

Luborsky, L., Singer, B. \& Luborsky, L. (1975) Comparative studies of psychotherapies: Is it true that "Everyone has won and all must have prizes"? Archives of General Psychiatry 32:995-1008. [MV]

Mace, R. (1998) The coevolution of human fertility and wealth inheritance strategies. Philosophical Transactions of the Royal Society B: Biological Sciences 353:389-97. [aDAC]

Mace, R. (2000) Evolutionary ecology of human life history. Animal Behaviour 59:1-10. [aDAC]

Mace, R. \& Sear, R. (2005) Are humans cooperative breeders? In: Grandmotherhood: The evolutionary significance of the second half of female life, ed. E. Voland, A. Chasiotis \& W. Schiefenhövel, pp. 143-59). Rutgers University Press. [aDAC]

Madrigal, L. \& Melendez-Obando, M. (2008) Grandmothers' longevity negatively affects daughters' fertility. American Journal of Physical Anthropology 136:223-29. [aDAC]

Mangen, D. J., Bengtson, V. L. \& Landry, P. H., Jr., eds. (1988) Measurement of intergenerational relations. Sage. [rDAC]

Manton, K. G., Gu, X. \& Lowrimore, G. R. (2008) Cohort changes in active life expectancy in the U.S. elderly population: Experience from 1982-2004 national long-term care survey. The Journals of Gerontology. Series B: Psychological Sciences and Social Sciences 63B:S269-81. [aDAC]

Marks, G. N. (2006) Family size, family type and student achievement: Crossnational differences and the role of socioeconomic and school factors. Journal of Comparative Family Studies 37:1-24. [arDAC]

Masvie, H. (2006) The role of Tamang mothers-in-law in promoting breast feeding in Makwanpur District, Nepal. Midwifery 22:23-31. [aDAC]

Mathews, P. S. \& Sear, R. (in preparation) Kin orientation and the timing of first birth: Evidence from the British Household Panel Survey. [RS]

Maxwell, R. J., Silverman, P. \& Maxwell, E. K. (1984) The motive for geronticide. In: Aging and the aged in the Third World: Part 1. Studies in Third World societies, No. 22, ed. J. Sokolovsky, pp. 67-84. William and Mary College. [MG]

Maynard Smith, J. (1964) Group selection and kin selection. Nature 201:114547. [aDAC]

Mayr, E. (1961) Cause and effect in biology. Science 134:1501-506. [aDAC]

McClellan, W. M., Stanwyck, D. J. \& Anson, C. A. (1993) Social support and subsequent mortality among patients with end-stage renal disease. Journal of the American Society of Nephrology 4:1028-34. [aDAC]

McDonald, P. (2000) Gender equity, social institutions and the future of fertility. Journal of Population Research 17:1-16. [RK]

McHenry, H. M. (1994) Behavioral ecological implications of early hominid body size. Journal of Human Evolution 27:77-87. [aDAC]

Medawar, P. B. (1952) An unsolved problem of biology. H. K. Lewis. [aDAC]

Meehan, C. L. (2005) The effects of residential locality on parental and alloparental investment among the Aka foragers of the Central African Republic. Human Nature 16:58-80. [aDAC]

Michalski, R. L. \& Shackelford, T. K. (2005) Grandparental investment as a function of relational uncertainty and emotional closeness with parents. Human Nature 16:293-305. [rDAC, AP]

Mills, T. L., Wakeman, M. A. \& Fea, C. B. (2001) Adult grandchildren's perceptions of emotional closeness and consensus with their maternal and paternal grandparents. Journal of Family Issues 22:427-55. [aDAC]

Ministry of Social Development, New Zealand (2009) The Social Report. Available at: http://www.socialreport.msd.govt.nz/people/fertility.html [RS]
Mitchell, W. (2007) Research review: The role of grandparents in intergenerational support for families with disabled children: A review of the literature. Child and Family Social Work 12:94-101. [aDAC]

Modin, B. \& Fritzell, J. (2009) The long arm of the family: Are parental and grandparental earnings related to young men's body mass index and cognitive ability? International Journal of Epidemiology 38:724-32. [aDAC]

Monserud, M. A. (2008) Intergenerational relationships and affectual solidarity between grandparents and young adults. Journal of Marriage and Family 70:182-95. [arDAC]

Moore, M. R. \& Brooks-Gunn, J. (2002) Adolescent parenthood. In: Handbook of parenting, vol. 3: Being and becoming a parent, 2nd edition, ed. M. H. Bornstein, pp. 173-214. Erlbaum. [aDAC]

Morris, J. K. \& Alberman, E. (2009) Trends in Down's syndrome live births and antenatal diagnoses in England and Wales from 1989 to 2008: Analysis of data from the National Down Syndrome Cytogenetic Register. British Medical Journal 339:b3794. [DCB]

Murphy, M. J. \& Grundy, E. (2003) Mothers with living children and children with living mothers: The role of fertility and mortality in the period 1911-2050. Population Trends 112:36-44. [aDAC]

Murray, C. J. L. \& Lopez, A. D. (1997) Alternative projections of mortality and disability by cause, 1990-2020: Global Burden of Disease Study. The Lancet 349:1498-504. [aDAC]

Myrskylä, M., Kohler, H.-P. \& Billari, F. C. (2009) Advances in development reverse fertility declines. Nature 460:741-43. [aDAC, CAH, RK]

Nag, M., White, B. \& Peet, R. (1978) An anthropological approach to the study of the economic value of children in Java and Nepal. Current Anthropology 19:293-306. [KLK]

Nettle, D., Coall, D. A. \& Dickins, T. E. (2010) Birthweight and paternal involvement predict early reproduction in British women: Evidence from the British National Child Development Study. American Journal of Human Biology 22(2):172-79. [RS]

Nettle, D., Dickins, T. E. \& Coall, D. A. (in submission.) Patterns of physical and psychological development in future teenage mothers. [RS]

Newson, L., Postmes, T., Lea, S. E. G. \& Webley, P. (2005) Why are modern families small? Toward an evolutionary and cultural explanation for the demographic transition. Personality and Social Psychology Review 9(4):360 75. [RS $]$

Oakley, L., Doyle, P. \& Maconochie, N. (2008) Lifetime prevalence of infertility and infertility treatment in the UK: Results from a population-based survey of reproduction. Human Reproduction 23:447-50. [aDAC]

Oburu, P. O. (2005) Caregiving stress and adjustment problems of Kenyan orphans raised by grandmothers. Infant and Child Development 14:199-210. [aDAC]

Oburu, P. O. \& Palmerus, K. (2005) Stress related factors among primary and parttime caregiving grandmothers of Kenyan grandchildren. International Journal of Aging and Human Development 60:273-82. [aDAC]

Oeppen, J. \& Vaupel, J. W. (2002) Broken limits to life expectancy. Science 296:1029-31. [rDAC]

O'Hara, M. W. \& Swain, A. M. (1996) Rates and risk of postpartum depression: A meta-analysis. International Review of Psychiatry 8:37-54. [aDAC]

Okabayashi, H., Liang, J., Krause, N., Akiyama, H. \& Sugisawa, H. (2004) Mental health among older adults in Japan: Do sources of social support and negative interaction make a difference? Social Science and Medicine 59:2259-70. [aDAC]

Oyserman, D., Radin, N. \& Benn, R. (1993) Dynamics in a three-generational family: Teens, grandparents, and babies. Developmental Psychology 29:56472. [aDAC]

Packer, C., Tatar, M. \& Collins, A. (1998) Reproductive cessation in female mammals. Nature 392:807-11. [aDAC]

Paine, R. R. \& Boldsen, J. L. (2006) Paleodemographic data and why understanding Holocene demography is essential to understanding human life history evolution in the Pleistocene. In: The evolution of human life history, ed. K. Hawkes \& R. R. Paine, pp. 307-30. School of American Research Advanced Seminar Series. School of American Research Press. [rDAC]

Pallock, L. L. \& Lamborn, S. D. (2006) Beyond parenting practices: Extended kinship support and the academic adjustment of African-American and European-American teens. Journal of Adolescence 29:813-28. [aDAC]

Pashos, A. (2000) Does parental uncertainty explain discriminative grandparental solicitude? A cross-cultural study in Greece and Germany. Evolution and Human Behavior 21:97-109. [aDAC, AP, MV]

Pashos, A. (2009) Ergebnisse - Deutscher Alterssurvey. NAR-Kolleg zu Heidelberg. Zwischenberichte Januar-Juni 2009 [Results-German Aging Survey. Network Aging Research Kolleg of Heidelberg. Progress reports, JanuaryJune 2009], pp. 66-69. Unpublished research report. [AP]

Pashos, A. \& McBurney, D. H. (2008) Kin relationships and the caregiving biases of grandparents, aunts and uncles: A two-generational questionnaire study. Human Nature 19:311-30. [aDAC, AP] 
Patrick, J. H. \& Goedereis, E. A. (2009) The importance of context and the gain-loss dynamic for understanding grandparent caregiving. In: How caregiving affects development, ed. L. Shifren, pp. 169-90. American Psychological Association. [HAE]

Patterson, C. J., Hurt, S. \& Mason, C. D. (1998) Families of the lesbian baby boom Children's contact with grandparents and other adults. American Journal of Orthopsychiatry 68:390-99. [aDAC]

Paul, A. (2005) Primate predispositions for human grandmaternal behavior. In: Grandmotherhood: The evolutionary significance of the second half of female life, ed. E. Voland, A. Chasiotis \& W. Schiefenhövel, pp. 21-37. Rutgers University Press. [aDAC]

Pavard, S., Koons, D. N. \& Heyer, E. (2007) The influence of maternal care in shaping human survival and fertility. Evolution 61:2801-10. [aDAC]

Pavelka, M. S. M. \& Fedigan, L. M. (1991) Menopause: A comparative life history perspective. Yearbook of Physical Anthropology 34:13-38. [aDAC]

Peccei, J. S. (1995) The origin and evolution of menopause: The altriciality-lifespan hypothesis. Ethology and Sociobiology 16:425-49. [aDAC]

Peccei, J. S. (2001a) A critique of the grandmother hypotheses: Old and new. American Journal of Human Biology 13:434-52. [DCB, aDAC]

Peccei, J. S. (2001b) Menopause: Adaptation or epiphenomenon? Evolutionary Anthropology 10:43-57. [aDAC]

Penn, D. J. \& Smith, K. R. (2007) Differential fitness costs of reproduction between the sexes. Proceedings of the National Academy of Sciences USA 104:55358. [aDAC $]$

Perozek, M. G. (1998) A reexamination of the strategic bequest motive. Journal of Political Economy 106:423-45. [DC]

Pfau-Effinger, B. (2005) Welfare state policies and the development of care arrangements. European Societies 7:321-47. [RK]

Pittman, L. D. (2007) Grandmothers' involvement among young adolescents growing up in poverty. Journal of Research on Adolescence 17:89-116. [rDAC]

Poehlmann, J. (2005) Children’s family environments and intellectual outcomes during maternal incarceration. Journal of Marriage and Family 67:1275-85. [aDAC]

Pollet, T. V., Nelissen, M. \& Nettle, D. (2009) Lineage based differences in grandparental investment: Evidence from a large British cohort study. Journal of Biosocial Science 41:355-79. [arDAC]

Pollet, T. V., Nettle, D. \& Nelissen, M. (2006) Contact frequencies between grandparents and grandchildren in a modern society: Estimates of the impact of paternity uncertainty. Journal of Cultural and Evolutionary Psychology 4:203-13. [aDAC]

Polley, D. C., Spicer, M. T., Knight, A. P. \& Hartley, B. L. (2005) Intrafamilial correlates of overweight and obesity in African-American and Native-American grandparents, parents, and children in rural Oklahoma. Journal of the American Dietetic Association 105:262-65. [rDAC]

Pope, S. K., Whiteside, I., Brooks-Gunn, J., Kelleher, K. J., Rickert, V. I., Bradley, R. H., \& Casey, P. H. (1993) Low-birth-weight infants born to adolescent mothers. Effects of coresidency with grandmother on child-development. Journal of the American Medical Association 269:1396-400. [aDAC]

Portegijs, W., Cloin, M., Ooms, I. \& Eggink, E. (2006) Hoe het werkt met kinderen (How it works with children). SCP (The Netherlands' Institute for Social Research). [RK $]$

Post, S. G. (2005) Altruism, happiness, and health: It's good to be good. International Journal of Behavioral Medicine 12:66-77. [aDAC]

Presser, H. B. (1989) Some economic complexities of child care provided by grandmothers. Journal of Marriage and Family 51:581-91. [aDAC]

Preston, S. O. \& de Waal, F. B. M. (2002) Empathy: Its ultimate and proximate bases. Behavioral and Brain Sciences 25:1-72. [aDAC]

Pruett-Jones, S. G. \& Lewis, J. J. (1990) Habitat limitation and sex ratio promote delayed dispersal in Superb fairy-wrens. Nature 348:541-42. [BIS]

Quenqua, D. (2009) One rude turn deserves a swat. New York Times, Sunday Styles Section, November 15, 2009, p. 1. [EF]

Reitzes, D. C. \& Mutran, E. J. (2004) Grandparenthood: Factors influencing frequency of grandparent-grandchildren contact and grandparent role satisfaction. The Journals of Gerontology. Series B: Psychological Sciences and Social Sciences 59B:S9-S16. [aDAC]

Robbins, M. S., Briones, E., Schwartz, S. J., Dillon, F. R. \& Mitrani, V. B. (2006) Differences in family functioning in grandparent and parent-headed households in a clinical sample of drug-using African American adolescents. Cultural Diversity and Ethnic Minority Psychology 12:84-100. [aDAC]

Robinson Sullivan, R., Lee, R. \& Kramer, K. (2008) Counting women’s labor: A reanalysis of children's net productivity in Mead Cain's Bangladeshi village. Population Studies 62:25-38. [KLK]

Rogers, A. R. (1993) Why menopause? Evolutionary Ecology 7:406-20. [aDAC]

Romaniuk, A. (1980) Increase in natural fertility during the early stages of modernization: Evidence from an African case study, Zaire. Population Studies 34:293-310. [KLK]
References/Coall \& Hertwig: Grandparental investment

Rose, H. \& Rose, S. (2001) Alas poor Darwin: Arguments against evolutionary psychology. Vintage. [aDAC]

Rosman, E. A. \& Yoshikawa, H. (2001) Effects of welfare reform on children of adolescent mothers: Moderation by maternal depression, father involvement, and grandmother involvement. Women and Health 32:253-90. [rDAC]

Ross, M. E. T. \& Aday, L. A. (2006) Stress and coping in African American grandparents who are raising their grandchildren. Journal of Family Issues 27:912-32. [aDAC]

Rossi, A. S. \& Rossi, P. H. (1990) Of human bonding: Parent-child relations across the life course. Aldine de Gruyter. [aDAC]

Runyan, D. K., Hunter, W. M., Socolar, R. R., Amaya-Jackson, L., English, D., Landsverk, J., Dubowitz, H., Browne, D. H., Bangdiwala, S. I. \& Mathew, R. M. (1998) Children who prosper in unfavorable environments: The relationship to social capital. Pediatrics 101:12-18. [aDAC]

Russell, R. J. H. \& Wells, P. A. (1987) Estimating paternity confidence. Ethology and Sociobiology 8:215-20. [MV]

Ryff, C. D., Lee, Y. H., Essex, M. J. \& Schmutte, P. S. (1994) My children and me: Midlife evaluations of grown children and of self. Psychology and Aging 9:195-205. [CAH]

Salmon, C. A. \& Daly, M. (1998) Birth order and familial sentiment: Middleborns are different. Evolution and Human Behavior 19:299-312. [aDAC]

Sanfey, A. G., Rilling, J. K., Aronson, J. A., Nystrom, L. E. \& Cohen, J. D. (2003) The neural basis of economic decision-making in the ultimatum game. Science 300:1755-1758. [aDAC]

Scannapiego, M. \& Hegar, R. L. (2002) Kinship care providers: Designing an array of supportive services. Child and Adolescent Social Work Journal 19:31527. [HAE]

Schniter, E. (2009) Why old age: Non-material contributions and patterns of aging among older adult Tsimane'. Doctoral dissertation, Department of Anthropology, University of California, Santa Barbara. [MG]

Scholl Perry, K. (1996) Relationships among adolescents' ego development, their academic achievement, and the amount of their contact with and social distance from grandparents. Doctoral dissertation, Department of Psychology, Pace University, New York. Dissertation Abstracts International, DAI-B 57/ 08, p. 5391. (AAT 9701161) [aDAC]

Schölmerich, A., Leyendecker, B., Citlak, B., Miller, A. \& Harwood, R. (2005) Variability of grandmother's roles. In: Grandmotherhood: The evolutionary significance of the second half of female life, ed. E. Voland, A. Chasiotis \& W. Schiefenhövel, pp. 277-92. Rutgers University Press. [aDAC]

Schröder-Butterfill, E. (2004) Inter-generational family support provided by older people in Indonesia. Ageing and Society 24:497-530. [aDAC]

Schultz, N. W. (1980) A cognitive-developmental study of the grandchild-grandparent bond. Child Study Journal 10:7-26. [aDAC]

Schwartz, J. \& Waldrop, J. (1992) The growing importance of grandparents if you're marketing products and services for children and young adults. American Demographics 14:10-11. [aDAC]

Sear, R. (2008) Kin and child survival in rural Malawi: Are matrilineal kin always beneficial in a matrilineal society? Human Nature 19:277-93. [arDAC, BIS]

Sear, R. \& Mace, R. (2008) Who keeps children alive? A review of the effects of kin on child survival. Evolution and Human Behavior 29:1-18. [arDAC, RH, BIS]

Sear, R., Mace, R. \& McGregor, I. A. (2000) Maternal grandmothers improve nutritional status and survival of children in rural Gambia. Proceedings of the Royal Society B: Biological Sciences 267:1641-47. [arDAC, BIS]

Sear, R., Steele, F., McGregor, I. A. \& Mace, R. (2002) The effects of kin on child mortality in rural Gambia. Demography 39(1):43-63. [BIS]

Shadish, W. R. \& Sweeney, R. B. (1991) Mediators and moderators in metaanalysis: There's a reason why we don't let dodo birds tell us which psychotherapies should have prizes. Journal of Consulting and Clinical Psychology 59:883-93. [MV]

Shanley, D. P. \& Kirkwood, T. B. L. (2001) Evolution of the human menopause BioEssays 23:282-87. [aDAC]

Shanley, D .P., Sear, R., Mace, R. \& Kirkwood, T. B. L. (2007) Testing evolutionary theories of menopause. Proceedings of the Royal Society of London B: Biological Sciences 274:2943-49. [aDAC]

Shanok, A. F. \& Miller, L. (2007) Stepping up to motherhood among inner-city teens. Psychology of Women Quarterly 31:252-61. [aDAC]

Sherman, P. W. (1998) The evolution of menopause. Nature 392:759-60. [aDAC]

Silk, J. B. (2004) Practicing Hamilton's rule: Kin selection in primate groups. In Cooperation in primates and humans, ed. P. Kappeler \& C. van Schaik, pp. 25-46. Springer Press. [KLK]

Silk, J. B., Brosnan, S. F., Vonk, J., Henrich, J., Povinelli, D. J., Richardson, A. S., Lambeth, S. P., Mascaro, J., \& Schapiro, S. J. (2005) Chimpanzees are indifferent to the welfare of unrelated group members. Nature 437:1357-59. $[\mathrm{aDAC}]$ 
Silverstein, M. \& Ruiz, S. (2006) Breaking the chain: How grandparents moderate the transmission of maternal depression to their grandchildren. Family Relations 55:601-12. [aDAC]

Skaznik-Wikiel, M., Tilly, J. C., Lee, H.-J., Niikura, Y., Kaneko-Tarui, T., Johnson, J. \& Tilly, J. L. (2007) Serious doubts over “eggs forever?". Differentiation 75:9399. [aDAC $]$

Sloan, F. A., Picone, G. \& Hoerger, T. J. (1997) The supply of children's time to disabled elderly parents. Economic Inquiry 35:295-308. [DC]

Smith, J. \& Goodnow, J. J. (1999) Unasked-for support and unsolicited advice: Age and the quality of social experience. Psychology and Aging 14:108-21. [CAH]

Smith, M. S. (1987) Evolution and developmental psychology: Toward a sociobiology of human development. In: Sociobiology and psychology: Ideas, issues, and applications, ed. C. Crawford, M. Smith \& D. Krebs pp. 225-52. Erlbaum. [aDAC]

Smith, M. S. (1988) Research in developmental sociobiology: Parenting and family behavior. In: Sociobiological perspectives on human development, ed. K. B MacDonald, pp. 271-92. Springer. [aDAC]

Smith, M. S. (1991) An evolutionary perspective on grandparent-grandchild relationships. In: The psychology of grandparenthood, ed. P. K. Smith, pp. 155-75. Routledge. [aDAC]

Smith, M. S., Kish, B. J. \& Crawford, C. B. (1987) Inheritance of wealth as human kin investment. Ethology and Sociobiology 8:171-82. [aDAC]

Smith, P. K. \& Drew, L. (2002) Grandparenthood. In: Handbook of parenting: Vol. 3. Being and becoming a parent, 2 nd edition, ed. M. H. Bornstein, pp. 141-72 Erlbaum. [aDAC]

Smith, P. K. \& Drew, L. (2004) Grandparenting and extended support networks. In: Sage handbook of parenting, ed. M. Hohhughi \& N. Long, pp. 146-59. Sage. [aDAC]

Spieker, S. J. \& Bensley, L. (1994) Roles of living arrangements and grandmother social support in adolescent mothering and infant attachment. Developmental Psychology 30:102-11. [aDAC]

Stack, C. B. (1975) All our kin: Strategies for survival in a Black community. Harper \& Row. [aDAC]

Stecklov, G. (1999) Evaluating the economic returns to childbearing in Côte d'Ivoire. Population Studies 53:1-17. [KLK]

Steinbach, I. \& Henke, W. (1998) Grosselterninvestment - eine empirische interkulturelle Vergleichsstudie [Grandparental investment - An empirical cross-cultural comparative study]. Anthropologie 36:293-301. [aDAC, AP, MV]

Stevens, J. H. (1988) Social support, locus of control, and parenting in three lowincome groups of mothers: Black teenagers, black adults, and white adults. Child Development 59:635-42. [aDAC]

Strassmann, B. I. \& Clarke, A. L. (1998) Ecological constraints on marriage in rural Ireland. Evolution and Human Behavior 19:33-55. [BIS]

Strassman, B. I. \& Dunbar, R. I. (1999) Human evolution and disease: Putting the Stone Age in perspective. In: Evolution in health and disease, ed. S. C. Stearns, pp. 91-101). Oxford University Press. [aDAC]

Strassmann, B. I. \& Gillespie, B. (2002) Life-history theory, fertility and reproductive success in humans. Proceedings of the Royal Society of London B 269:553-62. [BIS]

Strassmann, B. I. \& Kurapati, N. (in preparation) Grandparenting, menopause, and cooperative breeding in humans: A reassessment. [BIS]

Strazdins, L., Clements, M. S., Korda, R. J., Broom, D. H. \& D’Souza, R. M. (2006 Unsociable work? Nonstandard work schedules, family relationships, and children's well-being. Journal of Marriage and Family 68:394-410. [CAH]

Streib, G. F. (1958) Family patterns in retirement. Journal of Social Issues 14:4660. [aDAC]

Sugiyama, L. S. (2004) Illness, injury, and disability among Shiwiar forager-horticulturalists: Implications of health-risk buffering for the evolution of human life history. American Journal of Physical Anthropology 123:371-89. [aDAC]

Suomi, S. J. (2003) Gene-environment interactions and the neurobiology of social conflict. Annals of the New York Academy of Sciences 1008:132-39. [AP]

Surkan, P. J., Ryan, L. M., Carvalho Vieira, L. M., Berkman, L. F. \& Peterson, K. E. (2007) Maternal social and psychological conditions and physical growth in low-income children in Piauí, Northeast Brazil. Social Science and Medicine 64:375-88. [aDAC]

Szinovacz, M. E. (1998a) Grandparents today: A demographic profile. The Gerontologist 38:37-52. [arDAC]

Szinovacz, M. E. (1998b) Research on grandparenting: Needed refinements in concepts, theories, and methods. In: Handbook on grandparenthood, ed. M. E. Szinovacz, pp. 257-88. Greenwood Press. [aDAC]

Tesch-Römer, C., Wurm, S., Hoff, A. \& Engstler, H. (2002) Die zweite Welle des Alterssurveys. Erhebungsdesign und Instrumente [The second wave of the Ageing Survey: Research design and instruments]. Deutsches Zentrum für Altersfragen Diskussionspapiere, Nr. 35. DZA. Available at: http:// www.dza.de/nn_12036/SharedDocs/Publikationen/
Alterssurvey__Instrumente,templateId = raw, property $=$ publicationFile.pdf/Alterssurvey_Instrumente.pdf [AP]

Thiele, D. M. \& Whelan, T. A. (2008) The relationship between grandparent satisfaction, meaning, and generativity. International Journal of Aging and Human Development 66:21-48. [rDAC]

Thomas, M. \& Avery, V. (1997) Infant feeding in Asian families: Early feeding practices and growth. The Stationery Office, London. [aDAC]

Thompson, R. A., Tinsley, B. R., Scalora, M. J. \& Parke, R. D. (1989) Grandparents' visitation rights: Legalizing the ties that bind. American Psychologist 44:121722. [aDAC $]$

Tinsley, B. R. \& Parke, R. D. (1984) Grandparents as support and socialization agents. In: Beyond the dyad, ed. M. Lewis, pp. 161-94. Plenum. [aDAC]

Tinsley, B. J. \& Parke, R. D. (1987) Grandparents as interactive and social support agents for families with young infants. International Journal of Aging and Human Development 25:259-77. [arDAC]

Tolson, T. F. J. \& Wilson, M. N. (1990) The impact of two- and three-generational black family structure on perceived family climate. Child Development 61:416-28. [aDAC]

Tomlin, A. M. (1998) Grandparents' influences on grandchildren. In: Handbook on grandparenthood, ed. M. E. Szinovacz, pp. 159-70. Greenwood Press. [aDAC]

Tran, U. S., Fisher, M. L. \& Voracek, M. (2009) Spousal age differences and sex differences in life expectancy are confounders of matrilateral biases in kin investment. Basic and Applied Social Psychology 31:295-303. [MV]

Trivers, R. L. (1972) Parental investment and sexual selection. In: Sexual selection and the descent of man 1871-1971, ed. B. G. Campbell, pp. 136-79. Aldine. [arDAC]

Trivers, R. L. (1974) Parent-offspring conflict. American Zoologist 14:249-64. [rDAC, TWF]

Trivers, R. L. \& Willard, D. E. (1973) Natural selection of parental ability to vary the sex ratio of offspring. Science 179:90-92. [aDAC]

Turke, P. W. (1988) Helpers at the nest: Childcare networks on Ifaluk. In: Human reproductive behaviour: A Darwinian perspective, ed. L. Betzig, M. Borgerhoff Mulder \& P. Turke, pp. 173-88. Cambridge University Press. [aDAC, KLK]

Turke, P. W. (1989) Evolution and the demand for children. Population and Development Review 15:61-90. [RH, RS]

Turke, P. W. (1997) Hypothesis: Menopause discourages infanticide and encourages continued investment by Agnates. Evolution and Human Behavior 18:3-13. [aDAC]

Uhlenberg, P. (1996) Mortality decline in the twentieth century and supply of kin over the life course. The Gerontologist 36:681-85. [aDAC]

Uhlenberg, P. (2009) Children in an aging society. The Journals of Gerontology. Series B: Psychological Sciences and Social Sciences 64B:489-96. [aDAC]
[a.

Uhlenberg, P. \& Hammill, B. G. (1998) Frequency of grandparental contact with grandchild sets: Six factors that make a difference. The Gerontologist 38:27685. [aDAC $]$

Van Bavel, J. (2006) The effect of fertility limitation on intergenerational social mobility: The quality-quantity trade-off during the demographic transition. Journal of Biosocial Science 38:553-69. [arDAC]

van den Berg, P., Fawcett, T. W., Weissing, F. J. \& Buunk, A. P. (2009) A theoretical approach to the parent-offspring conflict over mate choice. European Human Behaviour and Evolution Association (EHBEA) meeting, April 6-8, 2009, St Andrews, UK. [TWF]

Victora, C. G. \& WHO Collaborative Study Team (2000) Effect of breastfeeding on infant and child mortality due to infectious diseases in less developed countries. A pooled analysis. WHO Collaborative Study Team on the Role of Breastfeeding on the Prevention of Infant Mortality. The Lancet 355:45155. [aDAC $]$

Vining, D. R. (1986) Social versus reproductive success: The central theoretical problem of human sociobiology. Behavioral and Brain Sciences 9:167-260. [rDAC]

Voland, E. \& Beise, J. (2002) Opposite effects of maternal and paternal grandmothers on infant survival in historical Krummhörn. Behavioral Ecology and Sociobiology 52:435-43. [aDAC, BIS]

Voland, E. \& Beise, J. (2005) "The husband's mother is the devil in house": Data on the impact of the mother-in-law on stillbirth mortality in historical Krummhörn (1750-1874) and some thoughts on the evolution of postgenerative female life. In: Grandmotherhood: The evolutionary significance of the second half offemale life, ed. E. Voland, A. Chasiotis \& W. Schiefenhövel, pp. 239-55. Rutgers University Press. [aDAC]

Voland, E., Chasiotis, A. \& Schiefenhövel, W. (2005) Grandmotherhood: A short overview of three fields of research on the evolutionary significance of postgenerative female life. In: Grandmotherhood: The evolutionary significance of the second half of female life, ed. E. Voland, A. Chasiotis \& W. Schiefenhövel, pp. 1-17. Rutgers University Press. [aDAC]

Voland, E., Siegelkow, E. \& Engel, C. (1991) Cost/benefit oriented parental investment by high status families: The Krummhörn case. Ethology and Sociobiology 12:105-118. [BIS] 
Vonk, J., Brosnan, S. F., Silk, J. B., Henrich, J., Richardson A. S., Lambeth, S. P., Schapiro, S. J. \& Povinelli, D. J. (2007) Chimpanzees do not take advantage of very low cost opportunities to deliver food to unrelated group members. Animal Behaviour 75:1757-70. [aDAC]

Voracek, M., Haubner, T. \& Fisher, M. L. (2008) Recent decline in nonpaternity rates A cross-temporal meta-analysis. Psychological Reports 103799-811. [MV]

Walker, R. S, Gurven, M., Burger, O. \& Hamilton, M. J. (2008) The trade-off between number and size of offspring in humans and other primates. Proceedings of the Royal Society of London B: Biological Sciences 275:827-34. [aDAC]

Warneken, F., Hare, B., Melis, A. P., Hanus, D. \& Tomasello, M. (2007) Spontaneous altruism by chimpanzees and young children. PLoS Biology 5:e184. [aDAC]

Watson, J. A., Rudolph, S. M. \& Lyons, J. L. (2005) African-American grandmothers as health educators in the family. International Journal of Aging and Human Development 60:343-56. [aDAC]

Weiss, K. M. (1981) Evolutionary perspectives on human aging. In: Other ways of growing old: Anthropological perspectives, ed. P. T. Amoss \& S. Harrell, pp. 25-58. Stanford University Press. [aDAC]

Weissman, M. M., Wickramaratne, P., Nomura, Y., Warner, V., Pilowsky, D. \& Verdeli, H. (2006) Offspring of depressed parents: 20 years later. American Journal of Psychiatry 163:1001-1008. [aDAC]

Werner, P., Lowenstein, A. \& Katz, R. (1998) The meaning of grandparenthood: A critical review and research agenda. Aging Clinical and Experimental Research 10:431-39. [aDAC]
WHO/UNICEF (1990) The Innocenti declaration on the protection, promotion and support of breastfeeding. Florence, Italy, August 1, 1990. Retrieved December 17, 2007 from: http://www.unicef.org/nutrition/ index_24807.html [aDAC]

Williams, G. C. (1957) Pleiotropy, natural selection, and the evolution of senescence. Evolution 11:398-411. [DCB, aDAC, BIS]

Wilson, D. S. \& Wilson, E. O. (2007) Rethinking the theoretical foundation of sociobiology. Quarterly Review of Biology 82:327-48. [aDAC]

Wolff, F. C. (2000) Transferts et redistribution familiale collective. Revue Économique 51:143-62. [aDAC]

Wood, S. \& Liossis, P. (2007) Potentially stressful life events and emotional closeness between grandparents and adult grandchildren. Journal of Family Issues 28:380-98. [aDAC]

Yong, F. (2006) Structural factors, types of grandparenting, and grandparents' wellbeing: Building causal mechanisms of grandparenting in an aging society. Doctoral dissertation, School of Social Welfare, State University of New York at Albany. Dissertation Abstracts International, DAI-A 67/10, p. 3935. (AAT 3239165). [aDAC]

Zervas, L. J. \& Sherman, M. F. (1994) The relationship between perceived parental favoritism and self-esteem. Journal of Genetic Psychology 169:281-88. [aDAC]

Zizzo, D. \& Oswald, A. (2001) Are people willing to pay to reduce others' incomes. Annales d'Economie et de Statistique 63/64:39-65. [EF] 